Division

Chemical Engineering

Division

Chemical Engineering

Division

Chemical Engineering

Division

Chemical Engineering

Division

Chemical Engineering

Division

Chemical Engineering

Division

Chemical Engineering

Division

Chemical Engineering

Division

Magnetically Responsive Micro

Particles for Targeted Drug and

Radionuclide Delivery

A Review of Recent Progress and Future Challenges

Chemical Engineering

Division

Chemical Engineering

Division

Chemical Engineering

Division

by M. D. Kaminski, L. Nuñez, A. N. Ghebremeskel, K. E. Kasza, P. F. Fischer, F.-C. Chang, T.-H. Chien, J. A. Eastman, J. R. Hull

A. J. Rosengart, L. Macdonald, Y. Xie, L. Johns

P. Pytel, and U. Häfeli

Chemical Engineering

Division

Chemical Engineering

Division

Chemical Engineering

Division

Chemical Engineering

Division

Chemical Engineering

Division

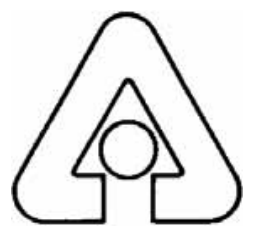

Argonne National Laboratory, Argonne, Illinois 60439

Operated by The University of Chicago

for the United States Department of Energy under Contract W-31-109-Eng-38

Chemical Engineering

Division

Chemical Engineering

Division

Chemical Engineering

Division

Chemical Engineering

Division 
Argonne National Laboratory, a U.S. Department of Energy Office of Science laboratory, is operated by The University of Chicago under contract W-31-109-Eng-38.

\footnotetext{
This report was prepared as an account of work sponsored by an agency of the United States Government. Neither the United States Government nor any agency thereof, nor The University of Chicago, nor any of their employees or officers, makes any warranty, express or implied, or assumes any legal liability or responsibility for the accuracy, completeness, or usefulness of any information, apparatus, product, or process disclosed, or represents that its use would not infringe privately owned rights. Reference herein to any specific commercial product, process, or service by trade name, trademark, manufacturer, or otherwise, does not necessarily constitute or imply its endorsement, recommendation, or favoring by the United States Government or any agency thereof. The views and opinions of document authors expressed herein do not necessarily state or reflect those of the United States Government or any agency thereof.
}

Available electronically at http://www.doe.gov/bridge

Available for a processing fee to U.S. Department of Energy and its contractors, in paper, from:

U.S. Department of Energy

Office of Scientific and Technical Information

P.O. Box 62

Oak Ridge, TN 37831-0062

phone: (865) 576-8401

fax: (865) 576-5728

email: reports@adonis.osti.gov 
ANL-03/28

ARGONNE NATIONAL LABORATORY

9700 South Cass Avenue

Argonne, IL 60439

\title{
Magnetically Responsive Microparticles for Targeted Drug and Radionuclide Delivery
}

\section{A Review of Recent Progress and Future Challenges}

\author{
M. D. Kaminski, A. N. Ghebremeskel, and L. Nuñez \\ Chemical Engineering Division \\ K. E. Kasza, F. Chang, and T.-H. Chien \\ Energy Technology Division \\ P. F. Fischer \\ Mathematics and Computer Science Division \\ J. A. Eastman \\ Materials Science Division \\ A. J. Rosengart, L. Macdonald, Y. Xie, L. Johns, and P. Pytel \\ The University of Chicago \\ Department of Neurology and Surgery (Neurosurgery) \\ U. O. Häfeli \\ The Cleveland Clinic Foundation \\ Department of Radiation Oncology
}

September 2003 


\section{CONTENTS}

A. REVIEW OF THE LITERATURE ..................................................................... 2

I. INTRODUCTION AND LITERATURE REVIEW …...................................... 2

a. Brief History of Magnetic Particle Targeting ....................................................... 4

II. PARTICLE DEVELOPMENT ...................................................................... 5

a. Magnetic Component ................................................................................ 6

b. Parameters Governing Toxicity and Vascular Survival...................................... 7

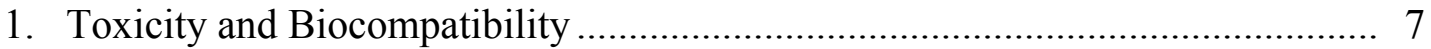

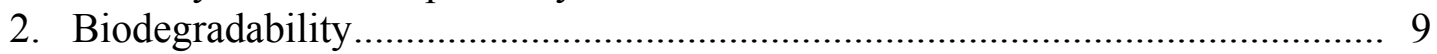

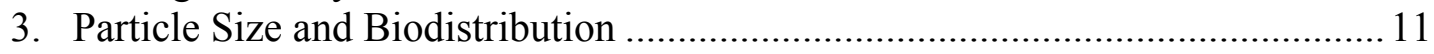

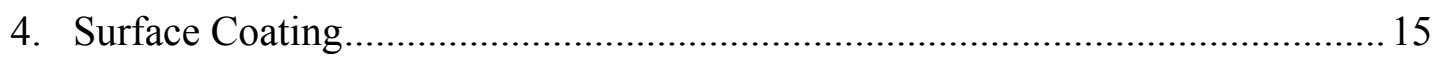

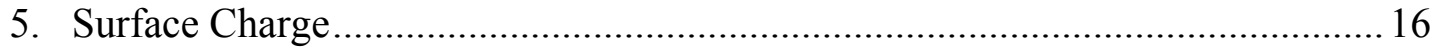

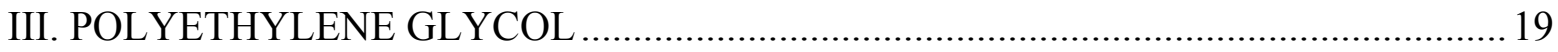

IV. METHODS FOR SYNTHESIS OF PARTICLES COMPOSED OF POLY(LACTIC ACID) AND POLY(LACTIC-CO-GLYCOLIC ACID) .....................22

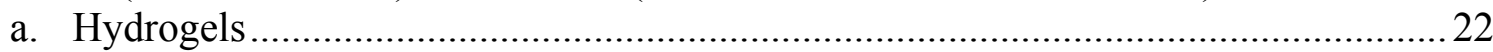

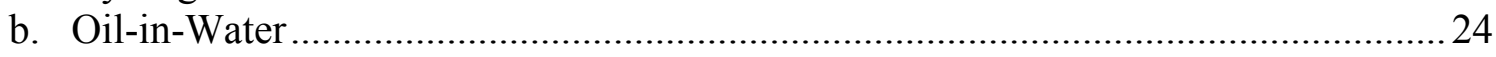

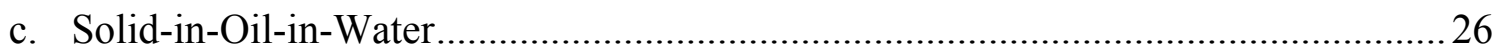

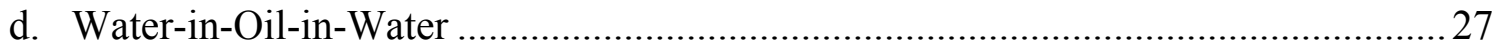

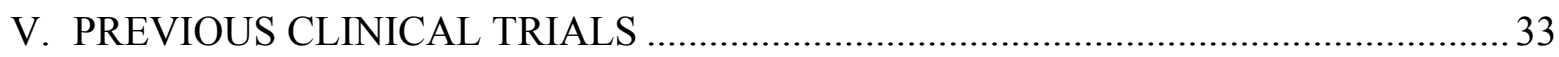

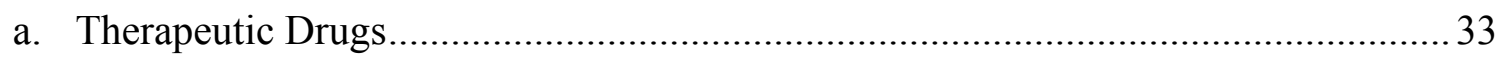

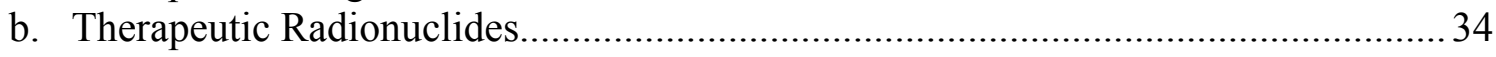

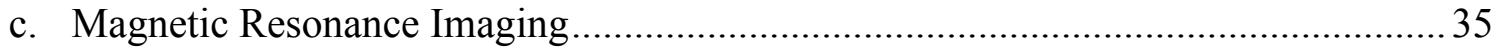

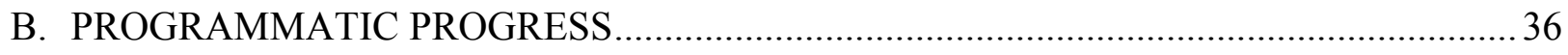

I. BIODEGRADABLE MICROPARTICLE SYNTHESIS ........................................ 36

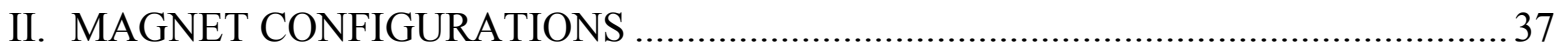

III. SYNTHESIS OF OXIDATION-PROTECTED AND SIZE-CONTROLLED IRON NANOPARTICLES 


\section{CONTENTS (Contd.)}

$\underline{\text { Page }}$

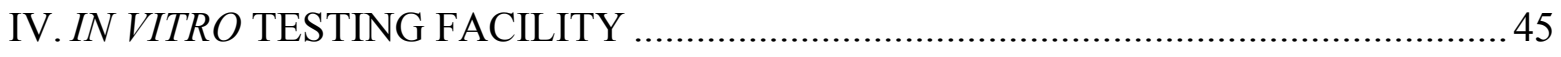

V. NUMERICAL MODELING OF MAGNETIC PARTICLES …................................ 48

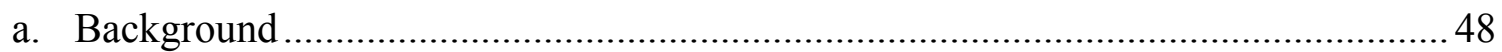

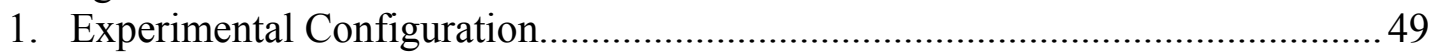

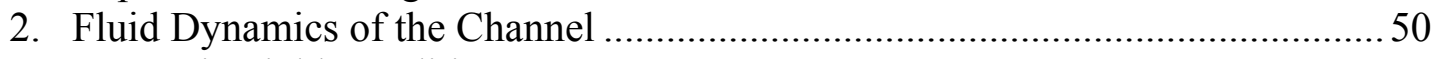

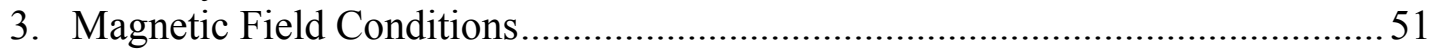

4. Particle Characteristics and Magnetic Force ................................................ 51

5. Dynamics of the Particle-Fluid Interaction........................................................ 53

b. Computation of Particle Trajectories ..................................................................... 53

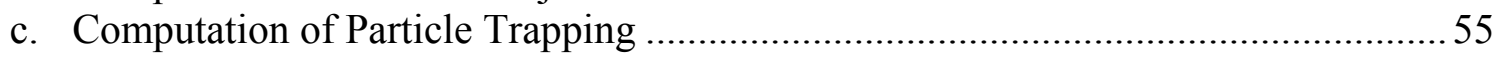

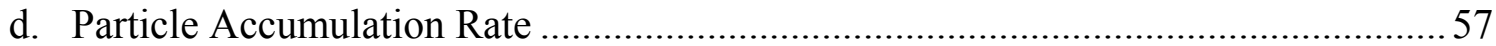

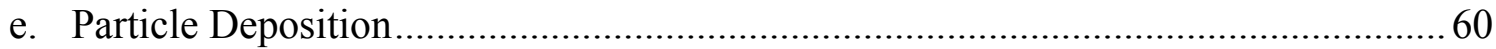

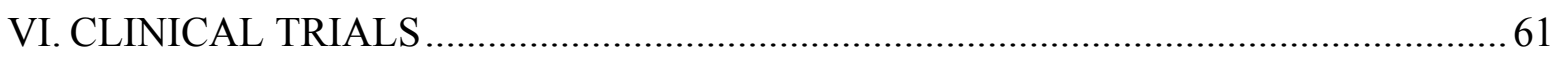

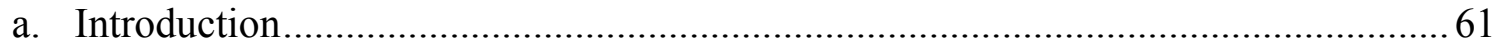

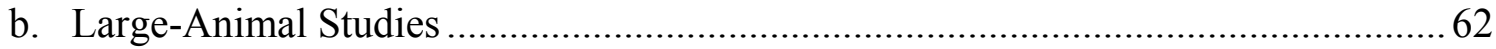

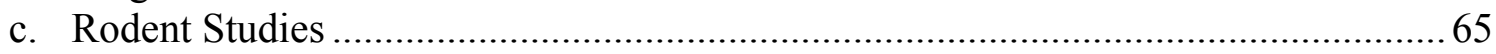

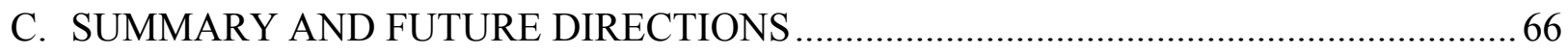

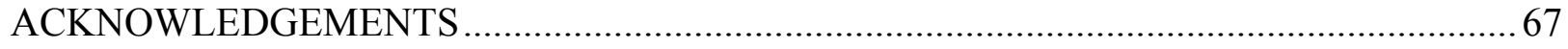

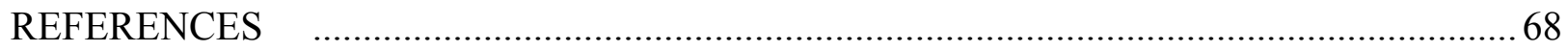

APPENDICES

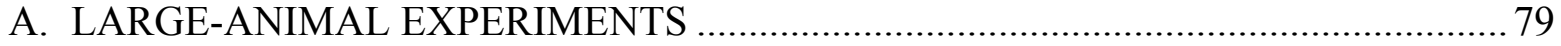

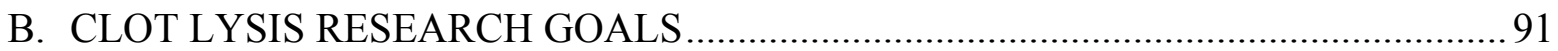

C. CANCER THERAPY RESEARCH GOALS .......................................................... 93 


\section{FIGURES}

1. Viability of EL-4 and DU-145 cell lines after addition of supernatants of magnetic microspheres and their controls $24 \mathrm{~h}$ after incubation at $37^{\circ} \mathrm{C}$.

2. Distribution of Ga-loaded liposomes composed of DSPC/Chol/PEG-DSPE or DSPC/Chol in mice with subcutaneously implanted C-26 colon carcinoma

3. Zeta potential of magnetic nanoparticles as function of basicity of their polymer matrix at constant $\mathrm{pH} 5.0 \pm 0.1$

4. Zeta potential of PS and PS-PEG2000 colloids in $0.001 \mathrm{M}$ ionic strength buffer with changing $\mathrm{pH}$

5. Zeta potential of magnetite, PLA, and mixed particles as function of $\mathrm{pH}$ in presence of $10^{-3} \mathrm{M} \mathrm{KNO}_{3}$

6. Interactions between protein and hydrophobic substrate with attached PEG chains. 20

7. Schematic representation of particle fabrication following an emulsionsolvent evaporation procedure

8. SEM image of PLA-based microspheres

9. Two-dimensional magnetic field strength profile for $\mathrm{NdFeB}$ magnets 38

10. Flux density contours for concept 1 38

11. Field lines for concept 2 39

12. Flux density for concept 2 .

13. Field lines for concept 3 40

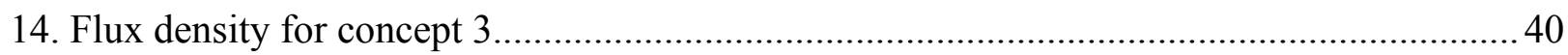

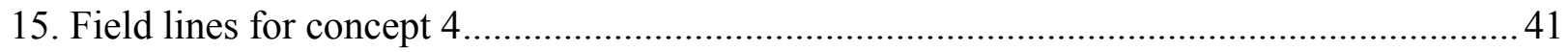

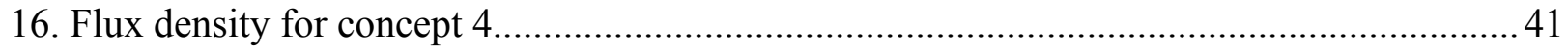

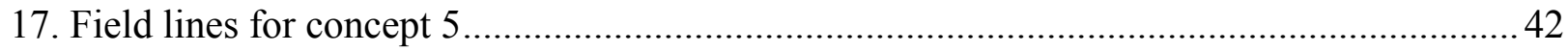

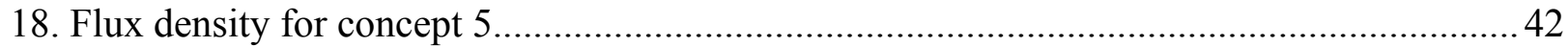




\section{FIGURES (Contd.)}

$\underline{\text { Page }}$

19 Flux density contours for concept 4 and concept 5 .

20. Schematic of chemical vapor condensation process for producing size-controlled carbon-coated iron nanoparticles dispersed in liquid.

21. Schematic drawing of apparatus for studying magnetic particle liquid-suspension behavior under influence of magnetic fields

22. Experimental apparatus for studying magnetic particle liquid-suspension behavior under influence of magnetic fields.

23. Magnetic particles held under magnet after 10 min of flow circulation

24. Comparison of particles of different magnetophoretic mobility.

25. Model configuration. 50

26. Measured B-field distribution

27. Velocity contours and particle capture zones at 50-, 100-, and $200-\mathrm{mL} / \mathrm{min}$ flow rates .......56

28. Predicted capture fractions for 7- $\mu \mathrm{m}$ particles at 50-, 100-, and 200-mL/min flow rates, with exponential fit

29. Predicted disposition pattern for $100-\mathrm{mL} / \mathrm{min}$ case 60

30. Comparison of current and proposed therapies for treating acute stroke 63

31. Histology-verified intra-arterial particle concentration at arterial region above surface magnet 


\section{TABLES}

1. In vitro degradation of drug loaded in $\mathrm{pH} 7.4$ phosphate buffer solution

2. Correlation between in vitro dissolution rate and in vitro degradation rate 10

3. Organ distribution of variously sized polystyrene microparticles in female beagle dogs $3.5 \mathrm{~h}$ after intravenous injection

4. Organ distribution of ${ }^{131}$ I-labeled gelatin nanoparticles and microparticles after intravenous administration in rats

5. Organ deposition for series of PLA:PEG micellar system in rat $3 \mathrm{~h}$ after intravenous injection

6. Biodistribution of radioactivity after intraperitoneal injection of ${ }^{90} \mathrm{Y}$-labeled magnetic microcapsules in mice

7. Biodistribution of uncoated and poloxamer-coated microspheres $24 \mathrm{~h}$ after intravenous administration into rats

8. Blood concentration of uncoated and poloxamer-coated microspheres $1 \mathrm{~h}$ after intravenous administration

9. Biodistribution of interferon- $\gamma$, free and in poly(L-lactide) microcapsules after oral administration in rats

10. Synthesis parameters for biodegradable particles

11. Physical characteristics of radionuclides of interest for targeted radiotherapy

12. Analysis of radiographic images of entire swine following ${ }^{99 \mathrm{~m}} \mathrm{Tc}-\mathrm{MTC}$ administration at $\mathrm{t}=60$ and $120 \mathrm{~min}$ from start of infusion

13. Particle transmission rates.

14. Remaining particle fraction after $4 \mathrm{~min}$ 


\section{ACRONYMS}

BBB

CFD

CVC

DNA

EG

EGFR

$\mathrm{H} \& \mathrm{E}$

HER2

IP

MA

MAA

MAbs

MPEG

MRI

MTC

MW

NGF

$\mathrm{O} / \mathrm{W}$

PBS

PEG

PEGMA

PEO

PLA

PLA-PEG

PLGA

PMAA

PV

PVA

RES

$\mathrm{SC}$

S/o/w

tPA

UV

$\mathrm{w} / \mathrm{o} / \mathrm{w}$

XRD
Blood-brain barrier

Computational fluid dynamics

Chemical vapor condensation

Deoxyribonucleic acid

Ethylene glycol

Epidermal growth factor receptors

Hematoxylin-Eosin

Human epidermal-growth-factor receptors

Intra-peritoneal

Monomethacrylate

Methacrylic acid

Monoclonal antibody

Monomethoxy-polyethylene-glycol

Magnetic resonance imaging

Magnetic targeting carrier

Molecular weight

Nerve growth factor

Oil-in-water

Phosphate Buffer Solution

Polyethylene glycol

Polyethylene glycol-monomethylether monomethacrylate

Polyethylene oxide

Poly-D,L-lactide

Poly-D,L-lactide-poly(ethyleneglycol)

Poly(lactic-glycolic acid)

Poly(methacrylic acid)

Polyvalerolactone

Polyvinyl alcohol

Reticuloendothelial system

Sub-cutaneous

solid-in-oil-in-water

Tissue plasminogen activator

Ultraviolet (light)

water-in-oil-in-water

X-ray diffraction 


\section{EXECUTIVE SUMMARY}

We are currently investigating the use of magnetic particles-polymeric-based spheres containing dispersed magnetic nanocrystalline phases-for the precise delivery of drugs via the human vasculature. According to this review, meticulously prepared magnetic drug targeting holds promise as a safe and effective method of delivering drugs to specific organ, tissue or cellular targets. We have critically examined the wide range of approaches in the design and implementation of magnetic-particle-based drug delivery systems to date, including magnetic particle preparation, drug encapsulation, biostability, biocompatibility, toxicity, magnetic field designs, and clinical trials.

However, we strongly believe that there are several limitations with past developments that need to be addressed to enable significant strides in the field. First, particle size has to be carefully chosen. Micrometer-sized magnetic particles are better attracted over a distance than nanometersized magnetic particles by a constant magnetic field gradient, and particle sizes up to $1 \mu \mathrm{m}$ show a much better accumulation with no apparent side effects in small animal models, since the smallest blood vessels have an inner diameter of 5-7 $\mu \mathrm{m}$. Nanometer-sized particles $<70 \mathrm{~nm}$ will accumulate in organ fenestrations despite an effective surface stabilizer. To be suitable for future human applications, our experimental approach synthesizes the magnetic drug carrier according to specific predefined outcome metrics: monodisperse population in a size range of $100 \mathrm{~nm}$ to $1.0 \mu \mathrm{m}$, non-toxic, with appropriate magnetic properties, and demonstrating successful in vitro and in vivo tests.

Another important variable offering possible improvement is surface polarity, which is expected to prolong particle half-life in circulation and modify biodistribution and stability of drugs in the body. The molecules in the blood that are responsible for enhancing the uptake of particles by the reticuloendothelial system (RES) prefer to associate with hydrophobic surfaces. Accordingly, we will tackle this challenge by modifying the particles with hydrophilic coatings such as PEG or poloxamer (co-polymers containing hydrophobic polyoxypropylene segments and repetitive polyoxyethylene hydrophilic groups), which have a proven ability to mask recognition by the RES.

Modeling is needed to help optimize the performance of targeted magnetic-particle delivery, enhance its medicinal value, and expedite its medical application. To this end, scientists at Argonne National Laboratory, working with The University of Chicago and Cleveland Clinic Hospital, are working on an effective magnetic drug targeting system based on custom magnetic field designs coupled to a three-dimensional imaging platform that addresses all associated physical and theoretical problems.

Furthermore, while our clinical trial results are encouraging with regard to the tolerance and applicability of the system, more improvements must be made with respect to future study designs and systems being used.

Given the technical hurdles in developing this potentially important technology, we believe we have made great progress and that we have a strong developmental plan. 


\section{A. REVIEW OF THE LITERATURE}

\section{INTRODUCTION AND LITERATURE REVIEW}

Precise targeting of drugs to diseased cells or locations within organs defines the "magic bullet" in medical therapy, but has not been achieved by current drug delivery methods. Targeting occurs when the drug or drug vehicle can identify a specific molecular feature in the diseased area. In the 1980s the medical and popular community became excited by the projected benefits of monoclonal antibody (MAbs) treatment. However, the biochemistry of cancerous cells proved too similar to that of healthy cells and the hope of revolutionary advancement in targeted cancer treatment using MAbs never materialized. Since then, incremental advances have been made to develop improved targeted delivery modalities. For example, in some forms of breast cancer, human epidermal growth factor receptors (HER2) are overexpressed in the individual. The monoclonal antibody trastuzumab was designed to bind directly to HER2 and is the only targeted hormonal therapy in clinical practice (Miller et al. 2002). The combination of targeted hormonal therapy and more traditional chemotherapy and radiation therapy regimens has produced a synergistic effect that has increased patient outcome (Mendelsohn 2002). The breadth of cancers these targeting technologies can reach is impressive. Approximately two-thirds of all solid tumors are known to overexpress epidermal growth factor receptor (EGFR). The degree of EGFR expression is quite variable among tumor types, ranging from 80 to $100 \%$ in head and neck cancers, from 14 to $91 \%$ in breast cancers, and from 31 to $44 \%$ in bladder cancers. Moreover, it has been known for some time that the degree of tumor EGFR overexpression is inversely proportional to prognosis (i.e., those individuals whose tumors express higher levels of EGFR have a worse prognosis than those whose tumors express lower levels of EGFR). In 1983, Dr. John Mendelsohn (2002) first hypothesized that EGFR inhibition might be an appropriate target for research into new anticancer agents. As a result, a number of compounds known as small molecule inhibitors have been developed, among them IMC-C225, ZD1839, and OSI-779.

In colorectal cancer, randomized clinical trials demonstrated that the addition of CPT-11 (irinotecan) to a regimen of either 5-FU (bolus 5-fluorouracil) or infusional 5-FU with leucovorin increased the survival of patients with advanced colorectal carcinoma. Consequently, this triple-drug regimen has become the standard of care for this disease (Kane 2002). Gene therapy is also an exciting field. This process identifies the malignant tissues that have abnormal loss of deoxyribonucleic acid (DNA) and loss of tumor suppression function. Mochamuk (2002) proposed to restore the lost DNA sequences and/or increase tumor suppressor activity. For example, wild-type p53 expression is part of cell cycle regulation, and well known to cause apoptosis (programmed cell death) in cells where the DNA has been damaged. P-53-recombinant adenoviruses (Ad-p53) contain engineered DNA sequences that are transported directly to cellular targets. Cellular uptake of the viral capsule by the tumor cell causes the DNA material to be released directly into the damaged cell. The repaired DNA sequencing then causes the cell to undergo normal apoptosis instead of continued survival and mutation (Mochamuk 2002). 
All these treatment technologies still suffer, however, from a lack of specificity. It has long been recognized that there is great difficulty in constructing a chemical sensor that can discriminate the target cells from all other cells in the body. One way to circumvent the problem or reduce the importance of chemical specificity while improving targeting efficiency is to physically manipulate the position of the drug. One such method is the use of intra-arterial catheters that can be directed to a solid tumor or blood clot and dispense a MAbs-tagged chemotherapy drug or clot-lysis drug, respectively, greatly decreasing the amount of systemic drug dose and increasing the treatment efficacy. The introduction of magnetic particles in the 1970s produced another transport vehicle for drug delivery. These first attempts concentrated on delivering chemotherapeutics to superficial tumors to decrease side effects. Unfortunately, the magnetic particles available at that time were not well engineered and the effects of particle injection in the body were not well understood. The advent of advanced methods for particle preparation has resulted in a second look at the efficacy of magnetic particles in delivering not only chemotherapeutics but also other drugs to specific disease sites in the body.

The newer magnetic particles are composed of a magnetic core material (usually iron or iron oxide) that has been treated to retain drugs on its surface or internally. The particles are small enough $(<5 \mu \mathrm{m})$ to navigate the artery and capillary systems of the body, but larger particles can lodge into certain tissues. By configuring magnets externally (outside the body), the particles can be stopped in the bloodstream or pulled in a direction of flow until the particles are positioned at the disease site. For tumors, the particles are drawn into the blood supply arteries of the tumor and lodged in the tissue. Radioisotopes or chemotherapeutic drugs attached to the particles can irradiate the tumor cells or desorb from the particles and attack the tumor cells. Indeed, given the intricate network of blood vessels and capillaries in the human body, precise guidance of particles to capillary beds is prohibitive. A sophisticated and powerful magnet system capable of operating in parallel with an imaging scheme (e.g., magnetic resonance imaging or computed tomography) may be needed to target or concentrate the magnetic particles in all but the most accessible (e.g., a superficial target or an organ that displays other conditions such as enhanced blood flow, natural filters, or sheer mass that naturally accumulates the particles) sites in the body. Crude bar magnets have been determined to work well for superficial tumor sites and for targeting liver tumors, and offer a relatively inexpensive capital investment compared with superconducting magnet designs. The most advanced superconducting magnet system has been developed by Stereotaxis, Inc., for guiding catheters within the human vasculature. Their system combines "real-time imaging and image guidance techniques with digital workstations, to bring computer-integrated surgical automation" to the clinic [www.stereotaxis.com].

Within the Chemical Engineering Division at Argonne National Laboratory, researchers have studied the chemical properties of magnetic particles in retaining radionuclides and metals from process and waste streams for goals in radioactive waste management and pollution prevention. Based on this knowledge, a technique was proposed whereby magnetic particles are tagged with high neutron capture cross-section isotopes (e.g., ${ }^{10} \mathrm{~B}$ and ${ }^{157} \mathrm{Gd}$ ) and guided to solid brain tumors for irradiation. As an extension, an interdisciplinary team at Argonne comprising four separate divisions (Chemical Engineering, Materials Science, Energy Technology, and Mathematics and Computer Science) has been assembled to identify and explore methods for producing layered and coated micro and nanomagnetic particles for delivering drugs and radioisotopes to diseased 
tissue. We envision this technology working synergistically with monoclonal antibody therapy, gene therapy, or aiding the established radiotherapies and chemotherapies and increasing target specificity. This review describes the current understanding of the properties and capabilities of magnetic particles for medical applications, and our progress in this technology field.

\section{a. Brief History of Magnetic Particle Targeting}

Spherical, polymeric delivery vehicles are often called particles, capsules, or carriers and can range from several nanometers to tens of micrometers. The use of magnetic iron oxide in polymer matrices (magnetic nano- and microencapsulation) to impart a magnetic moment to the particles began in the 1970s in the field of biotechnology. Most of these studies focused on enzyme immobilization and isolation of a wide range of specific cell binding agents (Robinson et al. 1973, Kronick et al. 1978, Olsvik et al. 1994, Thomas and Landsdorp 1994, Van der Wolf et al. 1994), hyperthermia (Chen et al. 1992, Jordan et al. 1997, Shinkai et al. 1999), magnetic resonance imaging contrast enhancement (Weissleder et al. 1995, Jung and Jacobs 1995, Bulte et al. 1999), tissue specific release of therapeutic agents (Gupta and Hung 1994, Lübbe et al. 1996a, Kumar 2000), radioimmunoassay (Nye et al. 1976), and magnetic targeting of therapeutic radio isotopes (Hafeli et al. 1994).

In 1978, Widder et al. reported the first experimental demonstration of site-specific drug delivery by albumin microcapsules $(0.2-2.0 \mu \mathrm{m})$ containing both $\mathrm{Fe}_{3} \mathrm{O}_{4}$ particles $(10-20 \mathrm{~nm})$ and chemotherapeutic agents. The unique feature of these delivery techniques compared with other drug targeting systems is their ability to minimize carrier uptake by the reticuloendothelial system (RES). Conceptually, the intended drug and a suitable magnetically-active component (e.g., $\mathrm{Fe}_{3} \mathrm{O}_{4}, \gamma-\mathrm{Fe}_{2} \mathrm{O}_{3}$ ) are formulated into a pharmaceutically-stable carrier system. The carrier (microcapsule) is injected through the artery that nourishes the tumor tissue, in the presence of an external magnet that can generate sufficient field strength and gradient to confine the carrier to the target site. The magnetic field is directed onto the tumor for a period sufficient to allow the transfer of the carrier from the blood compartment to the extravascular target tissue. The drug is released from the carrier in a controlled manner and then exerts its pharmacological action at cellular and/or subcellular level in the tumor tissue without critically affecting the survival of normal tissue (Widder et al. 1978). This approach has since been studied by many other authors (Tsyb et al. 1983, Sako and Hirota 1986, Stark et al. 1988, Pouliquen et al. 1989, Gupta and Hung 1993), outlining the possibility of targeting magnetic microcapsules, liposomes and ghost cells containing various cytotoxic drugs. More recent developments on magnetic targeting of radiotherapeutic particles are discussed by Häfeli et al. (2001).

Although attempts have been made to develop many different magnetic drug targeting methods (Blanchard et al. 1975, Gupta and Hung 1993, Lübbe et al. 1996a, Häfeli et al. 1994, Grüttner et al. 1997, Babincova et al. 1999, Alexiou et al. 2001), very few have advanced to human trials. A unique example, one that has progressed to FDA-approved therapeutic applications, is the Magnetic Targeted Carrier (MTC) drug delivery platform (FeRx, Inc., San Diego, California). The MTCs, which are made using a high-energy milling process, range in diameter from 0.5 to 5 $\mu \mathrm{m}$ and contain very high amounts (up to $80 \%$ ) of metallic iron, with the remaining weight consisting of activated carbon (Rudge et al. 2000). 
With regard to magnetic guidance systems, the magnetic design parameters in biomedical systems have distinct magnetic field characteristics and are usually tailored to meet the needs of a particular protocol. For example, there are two broad categories of magnetic systems capable of non-contact manipulation - those that produce the guided migration of an implant through relatively compact media like the parenchyma of the brain, and those that control the movement of catheters through the pulsatile flow of vasculature. In either case, a magnetic probe inside the body (either permeable or permanent) is acted on by magnetic fields and gradients generated outside the body in order to produce the desired motion (Gillies et al. 1994). The physical principles underlying the design of magnetic manipulation instrumentation offer an interesting challenge in their own right. Magnetic fields and gradients, typically generated from outside the body, are used to produce forces and torques on an ensemble of magnetic particles or probe masses inside the body. Through control of the fields and gradients, the magnetic particles or the probe mass can be made to move through body cavities, the vasculature, or even bulk tissue organs like the brain. This capability would clearly be a breakthrough for the many novel experimental therapies. Thus, ongoing development of magnetic manipulation instruments holds great promise for minimally invasive techniques, especially in light of recent advances in allied technologies such as magnetic materials, superconductivity, etc.

Currently, the major obstacles to the early clinical use of magnetic manipulation instruments can be summarized as follows: (1) Lack of data suggesting easy large-scale production of magnetic carriers with uniform and reproducible pharmaco characteristics, (2) absence of convincing data identifying magnetic carrier systems as safe and biocompatible, including concerns of acute adverse events of agglomeration and vascular occlusion and long-term side effects from particle deposition, (3) lack of safe and precise magnetic guidance systems for accurate drug delivery, and (4) regulatory approval and the economics of the therapy (Lübbe et al. 1996b). In addition to these issues, Alexiou et al. (2000) raised concerns regarding the challenge associated with intratumoral magnetic particle concentration: (1) the particle-drug complex should deliver and release a sufficient amount of active drug agent; and (2) the method of injection should have good access to the tumor and should avoid clearance by other systems.

Recently, however, progress has been made in overcoming these barriers by identifying biodegradable magnetic carriers systems, safe drug administration methods and first-generation prototype magnetic guidance systems. Our review begins with the current state of knowledge in designing magnetic particles useful for therapeutic drug or radioisotope delivery to specific sites in the body. Then, we discuss areas in which there is immediate opportunity for technical advancement. Finally, we summarize our experimental progress to date in defining a suitable particle composition, magnetic field profile for active targeting, computer modeling of the integrated system, and in vivo test results.

\section{PARTICLE DEVELOPMENT}

There is a systematic classification of different biodegradable polymers most often reported for presentation of pharmaceutical microcapsules for human use; polymers are classified as natural (starch, dextran, albumin, etc) and synthetic (polyesters, polyamides, polylactide, polyglycolide, etc). Arshady and Monshipouri (1999) state that the degree to which a particular polymer may be suitable as microcapsule shell or matrix for a given drug is determined by properties such as 
melting and glass transition, ability to form gel, fiber or film, and solubility parameters. The polymer must also have a certain degree of compatibility with (affinity toward) the core material (e.g., iron oxide, drug) to be encapsulated.

\section{a. Magnetic Component}

Two methods are commonly used to prepare the magnetic oxides that are incorporated into polymeric spheres to create a magnetic particle - size reduction and chemical precipitation, each with a number of modifications. The first method involves extensive wet grinding of a magnetic powder in the presence of a surfactant. The proportion of dispersant used during the grinding process corresponds closely to a monolayer coating on the final particles (Pouliquen 2001). For preparation of magnetic particles by chemical precipitation, two main procedures have been described. The first is based on Lefort's experiments, and involves the addition of a strong base $\left(\mathrm{NaOH}\right.$ or $\left.\mathrm{NH}_{4} \mathrm{OH}\right)$ to a mixture of iron salts $\left(\mathrm{Fe}^{3+} / \mathrm{Fe}^{2+}=2\right)$ (Lefort 1869). To maintain the particle size in the small colloidal range, a peptization step is generally included in which particles are transferred from water to an organic liquid containing a stabilizer. The second procedure is that of Massart and is based on the production of stable ionic ferrofluids without the use of stabilizers (Massart 1981).

In general, all the mechanisms that are used to manufacture polymeric magnetic particles can be classified into two techniques, either entrapment or impregnation (Arshady 1993). The entrapment method produces particles with a magnetic core composed of dispersed nanoparticle clusters (5-50 nm per particle) or nanoparticles surrounded by a polymer matrix. Generally, the magnetic nanoparticles are suspended in the polymeric solution prior to cross-linking or solvent evaporation. In contrast, the impregnation method involves the incorporation of magnetic oxide particles within the pores of preformed microspheres with a homogenous precipitate of magnetic oxide particles within the whole particle (Schütt et al. 1999).

Dynal, Inc., uses the impregnation technique to make a type of particle that is widely used. These micrometer-sized porous particles are formed with styrene divinylbenzene by activated swelling. At the surface of the pores, oxidative groups are introduced. These particles are stirred in a solution containing ferric chloride. The $\mathrm{Fe}^{3+}$ ions enter the pores, react with the oxidative groups and precipitate there as iron hydroxide. On heating, iron hydroxide is transformed to maghemite $\left(\gamma-\mathrm{Fe}_{2} \mathrm{O}_{3}\right)$. Since the size of the maghemite particles is less than the critical domain size, the particles are superparamagnetic. The remaining pores are then sealed with polymeric materials. Finally, the polystyrene particles are coated with oligomeric compounds, forming a thin shell to which specific ligands can be linked. Some ligands, like antibodies, can be directly adsorbed on to the particle surface due to hydrophobic interactions, or they can be covalently coupled by first activating the hydroxyl group with toluene-4-sulfonyl chloride (tosylation).

Another example of the impregnation technique is magnetic polystyrene microcapsules. Polystyrene-coated magnetic particles are made by adding surface-chelating polystyrene nanoparticles to a solution of Fe(II) and Fe(III) salts, followed by precipitation of iron oxide on the particles (Thomassen et al. 1991). Similarly, magnetic liposomes are obtained by homogenizing iron oxide $(10-20 \mathrm{~nm})$ with docosanoic acid and phosphatidylcholine. These particles could be used for imaging abscesses by virtue of their phagocytosis around inflammatory cells (Chan et al. 1992). 
The entrapment technique was used by Grüttner and Teller to synthesize superparamagnetic polysaccharide nanoparticles $(200-400 \mathrm{~nm})$ with an iron oxide content of $75-80 \%$. The iron oxide aggregates were obtained by alkaline preparation of ferric and ferrous sulfate, followed by gentle washing to reduce the $\mathrm{pH}$ value of the iron oxide to neutral $\mathrm{pH}$. The dextran matrix was then synthesized by crosslinking dextran strands with silica nanostructures. Traces of glycerol dextran ethers were formed by reaction of dextran with glycidyloxypropyl(trimethoxy) silane. The formation of nanoscale silica structures was induced during coating of the iron oxide cores with the modified dextran by the addition of tetraethoxysilane in the presence of ammonia and ethanol. The size of the particles can be increased by increasing the concentrations of glycerol dextran during the ether sequence and tetraethoxysilane during the coating procedure (Grüttner and Teller 1999).

\section{b. Parameters Governing Toxicity and Vascular Survival}

A number of studies have focused on systemic delivery of nanoparticles carrying drugs and radioisotopes to specific sites in the body. Being systemically delivered requires that the nanoparticles circulate without occluding arteries, veins, or capillaries (related factors include particle size, surface morphology, and surface charge), and the physical components must be non-toxic, biocompatible, biodegradable, and acceptable for human medication in all other aspects (Arshaday and Monshipouri 1999).

\section{Toxicity and Biocompatibility}

Häfeli and Pauer (1999) state that the most important factors in determining the biocompatibility of magnetic particles are the magnetically responsive components, the size of the particles, their matrix substance, and the coatings used. Häfeli and Paur performed an investigation using an in vitro 3-[4,5-dimethylthiazol-2yl]-2,5-diphenyltetrazolium bromide assay that quantifies cell viability, cell survival, and cell growth to rapidly evaluate toxicity of magnetic particles. Two types of tumor cells were used: adherent human prostate cells (DU-145) and murine suspension lymphoma cells (EL-4). The test particles and controls include biodegradable poly(L-lactic acid) microspheres containing $30 \mathrm{wt} \%$ magnetite (=PLA-MMS), non-magnetic poly(L-lactic acid) microspheres (=PLA-MS), iron-carbon particles containing $20 \mathrm{wt} \%$ iron $(=\mathrm{FeC}-\mathrm{MS})$, charcoal, $40 \mathrm{wt} \%$ magnetite polystyrene microspheres (=PS-A), paramagnetic polystyrene latex particles (PS-B), non-magnetic polystyrene latex particles (=PS-C), and dextran-coated magnetic nanoparticles containing $30 \mathrm{wt} \%$ magnetite $(=\mathrm{CG} 34)$. As presented in Figure 1, all microspheres showed toxic effects, and no significant difference of viability (cell growth and cell survival) was seen for microspheres made from materials generally considered to be biocompatible. 


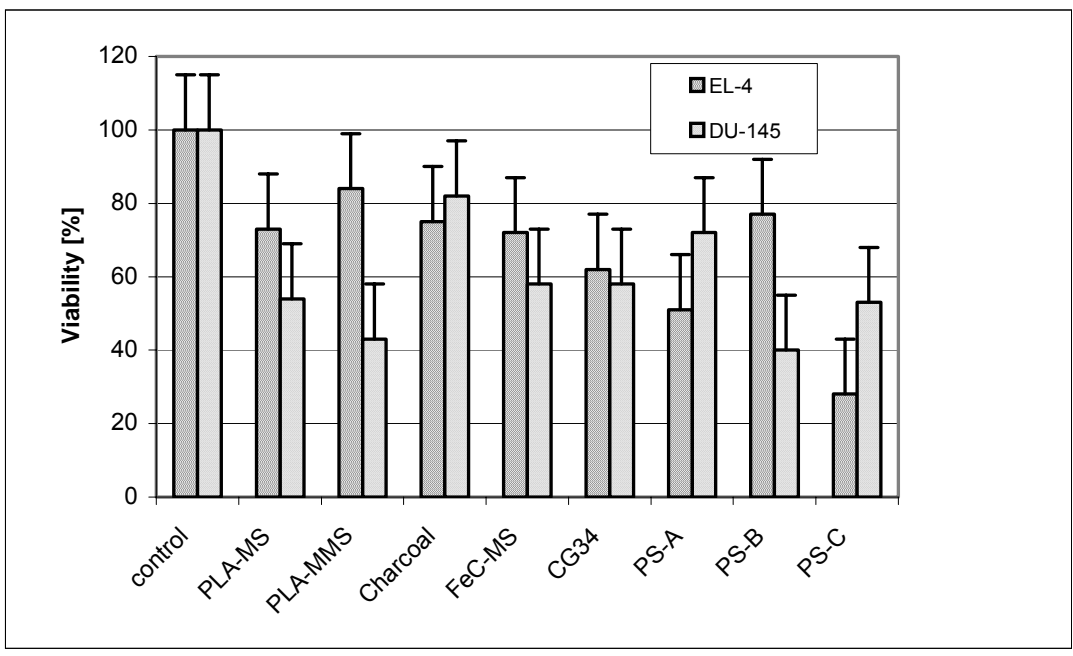

Figure 1. Viability of EL-4 and DU-145 cell lines after addition of supernatants of magnetic microspheres and their controls $24 \mathrm{~h}$ after incubation at $37^{\circ} \mathrm{C}$. Source: Häfeli (2003)

Other non-toxic polymeric nano- and microspheres have been described in the literature and comprise a category of natural and synthetic biopolymers. Biopolymers include but are not limited to poly(lactic acid), poly(lactic-co-glycolic acid), poly(ethylene glycol), albumin, and dextran. Biopolymers are chemically degraded at rates dependent on the particle size, surface properties, cross-linking density, and the molecular weight of the polymer (Arshady and Monshipouri 1999). The acute toxicity of several biopolymers has been evaluated (Häfeli and Pauer 1999) and suggests no ill effects due to the polymers. Instead, the polymers are degraded and metabolized into harmless fragments (Erbas et al. 2001). In our own work, histopathological examinations of lung, liver, brain, and spleen of a series of monkeys and rats exposed to systemic injections of magnetic particles showed no early toxicological changes (tissue changes, coagulation, extravasation) and no capillary obstructions in examined animal organs after adjustment of particle size and injection modus (Rosengart 2001).

The presence of magnetic particles incorporated within the polymeric matrix introduces a second source of potential toxicity. However, studies have shown (Okon et al. 1994, Pouliquen et al. 1991) that, in the long-term, the magnetite crystals are, in part, metabolized into increasing hepatic and splenic ferritin stores and, in part, incorporated into red blood cells. Thus, provided that the injected dose of magnetic iron is below the toxic dose threshold $(750 \mathrm{mg}$ iron for standard $\operatorname{man}^{1}$ ), it is safe. We estimate that about $100 \mathrm{mg}$ of nanoparticles will be injected to treat a typical subject, a fraction of which will be composed of iron (if there is $50 \%$ loading of the nanoparticle with elemental iron, then the patient may be exposed to $50 \mathrm{mg}$ of unbound iron). This amount of injected magnetite is much smaller than the dose leading to toxic iron effects.

Other sources of potential toxicity with synthetic polymers, and with regenerated and derivatized natural polymers are impurities associated with residual monomers, initiators, catalysts, solvents

\footnotetext{
${ }^{1} 10 \mathrm{mg} / \mathrm{kg}$ body mass or $750 \mathrm{mg}$ in standard man (Goldfrank 1998). Also, normal serum levels in blood are $80-180 \mu \mathrm{g} / \mathrm{dL}$ and action levels are $>500 \mu \mathrm{g} / \mathrm{dL}$.
} 
and stabilizers. Medical-grade polymers are prepared and purified according to specifically developed procedures with guaranteed levels of impurities, as stated in their data sheets. In the case of biodegradable polymers, however, the initial guarantee of purity is not a guarantee of non-toxicity during use (Arshady and Monshipouri 1999). Residual solvent is one example of impurity-related toxicity particularly relevant to polystyrene-based microparticles prepared by solvent extraction and evaporation. The question of solvent selection for these processes has been addressed in several publications (Mason et al. 1984, Arshady 1990, Bodmeier and McGinity 1988, and Gander et al. 1995). In the case of dichloromethane, the most convenient solvent for polystyrene microparticles, the acceptable limit set by the U.S. Pharmacopoeia is 500 ppm (Bleich and Mueller 1996). This limit can be safely reached by most conventional extraction and evaporation procedures, as well as by a recently reported procedure based on the use of supercritical carbon dioxide (Bleich and Mueller 1996). Bitz and Doelker (1996) have studied the effect of preparation conditions on residual solvent in biodegradable microcapsules.

According to Cleland (1995), dichloromethane (a pharmaceutical aid and solvent that metabolizes to carbon monoxide) is a suspected carcinogen and mutagen that has resulted in damage to liver and kidneys, nervous system disorders, and skin irritation. The $\mathrm{LD}_{50}$ for rats is $1.6 \mathrm{~g} / \mathrm{kg}$ (oral); for humans the $\mathrm{LDL}_{0}(375 \mathrm{mg} / \mathrm{kg}$ oral) produced a narcotic effect. Ethyl acetate, which is used as a pharmaceutical aid and a flavor additive, can irritate skin and eyes. Target organs are the liver, kidneys, as well as the blood and the nervous system. In mice, the $\mathrm{LD}_{50}$ has been shown to be $709 \mathrm{mg} / \mathrm{kg}$ intra-peritoneal (IP) and $4.1 \mathrm{~g} / \mathrm{kg}$ oral. For rats, the $\mathrm{LD}_{50}$ is 5.62 $\mathrm{g} / \mathrm{kg}$ oral and $5.0 \mathrm{~g} / \mathrm{kg}$ sub-cutaneous (SC). The $\mathrm{LD}_{50}$ for rabbits is $4.9 \mathrm{~g} / \mathrm{kg}$ oral and for cats 3.0 $\mathrm{g} / \mathrm{kg} \mathrm{SC}$.

\section{Biodegradability}

One of the desired features of therapeutic drug carriers, imaging agents, or other delivery entities administered intravenously to specific sites of action is biodegradability. Biodegradation of polymers in human medicine means polymer breakdown into resorbable (metabolizable) or excretable fragments - in ex vivo and in vitro test media, animal models, and the human bodythat represent, mimic or approximate conditions' (Arshady and Monshipouri 1999). Since prolonged use of non-biodegradable polymers can lead to accumulation in the body and possible chronic toxicity, they cannot be realistically considered safe for medicinal preparation (although they have benefit as experimental substrates).

Biodegradable materials degrade either through unzipping (the breaking of the last unit at the end of the chain, such as the removal of the last amino acid from a protein), or through random breaks along the chain. The main factor in their degradation is thought to be the properties of the medium (such as $\mathrm{pH}$, temperature, solvent and presence of biocatalysts), chemical composition (water, oxygen, ozone, halogenated compounds), electromagnetic radiation (visible light, ultraviolet (UV) light, $\gamma$-radiation), or any combination of these (Hasirci et al. 2001). 
Three low-molecular weight polyesters, poly(D,L-lactide) (PLA), poly(D,L-lactide-glycolide) (PLGA) and poly ( $\delta$-valerolactone) (PV), were used by Lin et al. to prepare water-soluble sodium diclofenac-loaded microspheres by (Lin et al. 2000). The degradation and dissociation rates (time-dependent decrease in average molecular weight) of the microspheres tested in vitro in $\mathrm{pH} 7.4 \mathrm{PBS}$ at $37^{\circ} \mathrm{C}$ are shown in Table 1 . The semi-log plots of average molecular weight vs. time were linear over the range of study. Thus, the degradation rate of all the microspheres was first-order kinetics and ranked in the order of PLGA $>$ PLA $>$ PV microspheres. Further, Table 2 shows the relationship between dissolution rate and degradation rate of polyester microspheres in $\mathrm{pH} 7.4$ phosphate buffer solution (PBS). Obviously, faster degradation of the polyester means faster dissolution of the drug from microspheres.

Table 1. In-vitro degradation of drug loaded in $\mathrm{pH} 7.4$ phosphate buffer solution.

\begin{tabular}{|c|c|c|c|}
\hline \multirow{2}{*}{ Time, days } & PV Number-average molecular weight (Mn) \\
\hline 0 & 4000 & PLA & PLGA \\
3 & 3500 & 4000 & 2000 \\
8 & 3400 & 3800 & 1000 \\
16 & 3000 & 3000 & 600 \\
22 & 3000 & 1000 & 230 \\
31 & 2800 & 700 & 20 \\
43 & 2600 & 410 & \\
65 & 2500 & 400 & \\
80 & 2000 & 140 & \\
\hline
\end{tabular}

Source: Modified from Lin et al. (2000)

Table 2. Correlation between in vitro dissolution rate and in vitro degradation rate.

\begin{tabular}{|c|c|c|}
\hline Microspheres & $\begin{array}{c}\text { Degradation Rate, } \\
\mathrm{x} 10^{-2} \mathrm{Mn} / \text { day }\end{array}$ & $\begin{array}{c}\text { Dissolution Rate, } \\
\% / \text { day }\end{array}$ \\
\hline PV & 1.50 & 0.15 \\
PLA & 6.50 & 0.25 \\
PLGA & 15.50 & 0.70 \\
\hline
\end{tabular}

Source: Modified from Lin et al. (2000)

Evidence of biodegradation and metabolization of iron from iron oxide crystals for magnetite dextran particles was demonstrated by Okon et al (1994). The results of transmission electron microscopy (TEM) of Kupffer cells following an intravenous (IV) administration show localization in lysosomes where they are biotransformed. Two main steps have been identified in biodegradation of the magnetite dextran particles: (1) decrease magnetic susceptibility of the tissue where the particles are concentrated, and (2) release of metabolized iron in the blood leading to increased hepatic or splenic ferritin concentration (Okon et al. 1994). The metabolized iron is then incorporated into red blood cells (Pouliquene et al. 1991). 


\section{Particle Size and Biodistribution}

The biodistribution of magnetic particles is generally studied by radiolabeled imaging and measurement of radioactivity in various tissues after intravenous administration of the labeled particles (Nye et al. 1976, Gupta and Hung 1993, Häfeli et al. 1994, Grüttner et al. 1997). Radiolabeling can be achieved by the addition of ${ }^{59} \mathrm{FeCl}_{3}$ during the formation of the magnetic particles. Other radioisotopes such as ${ }^{67} \mathrm{Ga},{ }^{99 \mathrm{~m}} \mathrm{Tc},{ }^{111} \mathrm{In},{ }^{125} \mathrm{I}$ and ${ }^{55} \mathrm{Fe}$ are also used for nuclear medicine studies (scintigraphy) and in-vitro experiments on cultured cells (Iannone et al. 1991).

The first-order biodistribution of nano- and microparticles is determined almost entirely by their size. It has been known for some time that following IV administration of microparticles larger than about $5 \mu \mathrm{m}$, the microparticles are almost entirely trapped in the lungs by arteriolar and capillary blockade (Table 3). Conversely, nanoparticles and microparticles smaller than about 3 $\mu \mathrm{m}$ are rapidly removed mainly by the Kupffer cells of the liver and spleen as a result of opsinization (protein adsorption) and engulfment by macrophages of the reticuloendothelial system (RES) (Table 4) (Yoshioka et al. 1981).

Table 3. Organ distribution of variously sized polystyrene microparticles in female beagle dogs $3.5 \mathrm{~h}$ after intravenous injection.

\begin{tabular}{|c|c|c|}
\hline \multirow{2}{*}{ Organ } & \multicolumn{2}{|c|}{ Particle Size } \\
\cline { 2 - 3 } & $3.4 \mu \mathrm{m}$ & $7.4 \mu \mathrm{m}$ and $11.6 \mu \mathrm{m}$ \\
\hline Liver & 73 & $<2.0$ \\
\hline Spleen & 10 & $<2.0$ \\
\hline Lungs & 12 & 93 \\
\hline Total & 95 & 97 \\
\hline
\end{tabular}

Source: Modified from Slack et al. (1981)

Table 4. Organ distribution (\% total dose) of ${ }^{131}$ I-labeled gelatin nanoparticles $(230-350 \mathrm{~nm})$ and microparticles $(12-19 \mu \mathrm{m})$ after intravenous administration in rats.

\begin{tabular}{|c|c|c|c|c|c|c|}
\hline \multirow{2}{*}{ Organ } & \multicolumn{3}{|c|}{ Nanoparticles } & \multicolumn{3}{c|}{ Microparticles } \\
\cline { 2 - 7 } & $10 \mathrm{~min}$ & $2 \mathrm{~h}$ & $24 \mathrm{~h}$ & $10 \mathrm{~min}$ & $2 \mathrm{~h}$ & $24 \mathrm{~h}$ \\
\hline Liver & 64 & 44 & 5.6 & 3.6 & 3.4 & 3.6 \\
\hline Spleen & 5.5 & 6.0 & 0.8 & 0.2 & 0.2 & 0.5 \\
\hline Lungs & 6.2 & 3.5 & 0.7 & 95 & 88 & 56 \\
\hline $\begin{array}{c}\text { Kidney + Heart + } \\
\text { Blood }\end{array}$ & 1.1 & 0.6 & 0.8 & 0.5 & 0.3 & 0.4 \\
\hline
\end{tabular}

Source: Modified from Yoshioka et al. (1981)

Following intra-arterial injection, microparticles are again cleared by the RES, while larger microparticles are sequestered in the first capillary bed encountered. As the microparticles begin to degrade, matrix products are released from the target circulation and gradually accumulate in the RES. This pattern of the particle deposition appears to be independent of the nature of the microparticles' polymeric matrix, such as chemical composition or hydrophilic character. Arshady and Monshipouri (1999) have shown a similar biodistribution data for microparticle types including poly(styrene-divinylbenzene), poly(acrylamide), albumin, gelatin, poly(alkyl cyanoacrylate), and poly(methyl methacrylate). 
Stolnik et al. (2001) reported in-vivo biodistribution of the micellar-like particle system, which is produced from poly(D,L-lactide), poly(ethyleneglycol) (PLA-PEG) copolymers, after intravenous administration to the rat. The size of the PEG chain was kept constant at $5 \mathrm{kDa}$, while the PLA size increased within a series from 2 to $25 \mathrm{kDa}$. The results revealed that, in an aqueous medium, the copolymers assembled into micellar-like structures, with the PLA segments forming the core and the PEG segments the surrounding corona. Table 5 shows that following intravenous administration to the rat model, micellar-like particles smaller than $\sim 70 \mathrm{~nm}$ accumulated in the liver, despite the fact that the PEG corona provided an effective steric stabilization effect. Micellar-like particles with a diameter of more than $\sim 70 \mathrm{~nm}$ exhibited prolonged systemic circulation and reduced liver uptake (Stolnik et al. 2001). This type of behavior was associated with nanoparticle leakage through fenestrations (holes) $<70 \mathrm{~nm}$ in diameter.

Table 5. Organ deposition for series of PLA:PEG (in $\mathrm{kDa}$ ) micellar systems in rat $3 \mathrm{~h}$ after intravenous injection.

\begin{tabular}{|c|c|c|c|c|c|}
\hline Organ & $\begin{array}{c}\text { PLA:PEG } \\
3: 5 \\
(26.6 \mathrm{~nm}) \\
\end{array}$ & $\begin{array}{c}\text { PLA:PEG } \\
6: 5 \\
(30.3 \mathrm{~nm}) \\
\end{array}$ & $\begin{array}{c}\text { PLA:PEG } \\
10: 5 \\
(42.7 \mathrm{~nm})\end{array}$ & $\begin{array}{c}\text { PLA:PEG } \\
13: 5 \\
(76.4 \mathrm{~nm}) \\
\end{array}$ & $\begin{array}{c}\text { PLA:PEG } \\
25: 5 \\
(71.2 \mathrm{~nm}) \\
\end{array}$ \\
\hline Liver & $82.3 \pm 8.9$ & $72.7 \pm 4.4$ & $72.2 \pm 1.9$ & $53.5 \pm 0.7$ & $57.5 \pm 1.3$ \\
\hline Spleen + Lungs & $2.5 \pm 0.2$ & $2.1 \pm 0.3$ & $2.4 \pm 0.4$ & $5.0 \pm 0.2$ & $6.4 \pm 0.5$ \\
\hline Kidney & $0.2 \pm 0.1$ & $0.1 \pm 0.0$ & $0.3 \pm 0.1$ & $0.8 \pm 0.1$ & $0.61 \pm 0.1$ \\
\hline Carcass & $5.0 \pm 1.7$ & $3.1 \pm 0.2$ & $7.9 \pm 1.2$ & $13.5 \pm 0.6$ & $10.1 \pm 0.5$ \\
\hline Blood & $3.2 \pm 0.9$ & $0.5 \pm 0.1$ & $2.9 \pm 0.1$ & $28.9 \pm 0.6$ & $24.1 \pm 0.6$ \\
\hline Recovery & $89.9 \pm 7.7$ & $77.9 \pm 4.9$ & $82.9 \pm 2.8$ & $72.9 \pm 0.4$ & $74.6 \pm 2.1$ \\
\hline
\end{tabular}

Source: Modified from Stolnik et al. (2001).

As reported by Häfeli et al. (1995), subcutaneous EL-4 murine lymphoma tumors (5 mm diameter) were grown in the belly area of mice and the biodistribution of microparticles studied. The animals were divided into non-magnet and magnet groups, and a round NdFeB magnet ( $2 \mathrm{~mm}$ thick, $10 \mathrm{~mm}$ diameter) was taped directly above the tumor in the magnet group. The magnetic field generated on top of this magnet was $0.12-0.16 \mathrm{~T}$. Radiolabeled magnetic microcapsules ( $2 \mathrm{mg}, 23 \pm 8 \mu \mathrm{m}, 11 \%$ magnetite, $1.1 \mathrm{MBq}{ }^{90} \mathrm{Y}$ ) were then injected into the peritoneal space at least $10 \mathrm{~mm}$ away from tumor. The animals were sacrificed after $24 \mathrm{~h}$, and radioactivity was measured (Table 6). In the control (non-magnet) group, microcapsules were distributed throughout the intraperitoneal area, with only $6 \pm 4 \%$ of injected radioactivity found in tumor, whereas in the magnetically targeted group, tumor-associated radioactivity was $73 \pm 32 \%$. 
Table 6. Biodistribution of radioactivity after intraperitoneal injection of ${ }^{90}$ Y-labeled magnetic microcapsules in mice $(n=10)$.

\begin{tabular}{|l|c|c|}
\hline \multirow{2}{*}{\multicolumn{1}{|c|}{ Organ }} & \multicolumn{2}{c|}{ Radioactivity per Gram Tissue (kBq) } \\
\cline { 2 - 3 } & Controls & Magnetically Targeted \\
\hline Heart + Lung & $3.8 \pm 7.1$ & $1.1 \pm 0.6$ \\
\hline Femur & $10.8 \pm 6.7$ & $4.6 \pm 4.8$ \\
\hline Small Intestine & $16.8 \pm 16.4$ & $15.1 \pm 25$ \\
\hline Spleen & $294.9 \pm 297$ & $31.2 \pm 24$ \\
\hline Kidney & $93.1 \pm 229$ & $2.5 \pm 1.1$ \\
\hline Liver + Blood & $21.0 \pm 11.7$ & $6.3 \pm 3.3$ \\
\hline Tumor Bed & $46.6 \pm 41$ & $972 \pm 683$ \\
\hline
\end{tabular}

Source: Modified from Häfeli et al. (1995).

Moghimi et al. (1991) reported biodistribution studies in groups of three male wistar rats (160 \pm $10 \mathrm{~g})$. The rats were injected intravenously with poloxamer-407- and poloxamer-908-coated polystyrene microspheres labeled with ${ }^{125}$ I via the tail vein. Blood samples of $20 \mu 1$ were taken from the tail vein at various time intervals. Animals were sacrificed at $24 \mathrm{~h}$ after injection and the activity associated with various organs of the RES measured. The results (Table 7) show that both hepatic and splenic uptake of uncoated microspheres are rapidly increased by increasing the size of microspheres from 60 to $250 \mathrm{~nm}$ and between these sites, hepatic uptake is predominant. Regardless of size, coating of microspheres with hydrophilic polymers dramatically reduced their uptake by the liver. This is also reflected in the high blood concentration of coated microspheres at $1 \mathrm{~h}$ post-injection (Table 8 ).

Table 7. Biodistribution of uncoated and poloxamer-coated microspheres $24 \mathrm{~h}$ after intravenous administration into rats.

\begin{tabular}{|c|c|c|c|c|}
\hline \multirow{2}{*}{$\begin{array}{c}\text { Microsphere } \\
\text { Size }(\mathrm{nm})\end{array}$} & \multicolumn{3}{|c|}{ \% of Injected Dose } \\
\cline { 2 - 5 } & Uncoated & $407-$ Coated & Uncoated & 407-Coated \\
\hline 60 & $38.2 \pm 7.1$ & $7.6 \pm 2.3$ & $0.3 \pm 0.0$ & $1.1 \pm 0.2$ \\
\hline 112 & $19.3 \pm 4.9$ & Not done & $1.2 \pm 0.6$ & Not done \\
\hline 150 & $60.7 \pm 1.8$ & $14.7 \pm 1.2$ & $2.6 \pm 0.5$ & $15.0 \pm 6.3$ \\
\hline 250 & $60.2 \pm 4.3$ & $14.0 \pm 4.3$ & $6.3 \pm 1.2$ & $39.5 \pm 1.7$ \\
\hline
\end{tabular}

Source: Modified from Moghimi et al. (1991).

The femoral uptakes were $0.05 \pm 0.05 \%$ and $0.5 \pm 0.1 \%$ of injected dose for uncoated and all polymeric coated microspheres, respectively, for all sizes tested. 
Table 8. Blood concentration of uncoated and poloxamer-coated microspheres $1 \mathrm{~h}$ after intravenous administration.

\begin{tabular}{|l|c|c|}
\hline \multirow{2}{*}{$\begin{array}{c}\text { Microsphere } \\
\text { Size }(\mathrm{nm})\end{array}$} & \multicolumn{2}{|c|}{ \% of Injected Dose } \\
\cline { 2 - 3 } & \multicolumn{2}{|c|}{$1 \mathrm{~h}$} \\
\hline 60 & Uncoated & 407-Coated \\
\hline 150 & $3.3 \pm 0.7$ & $37.1 \pm 14.2$ \\
\hline 250 & $3.3 \pm 3.3$ & $57.6 \pm 13.9$ \\
\hline
\end{tabular}

Source: Modified from Moghimi et al. (1991).

An interesting study by Eyles and colleagues reports the uptake and fate of free and PLAmicroencapsulated interferon following oral administration of (3-[ $\left.{ }^{125} \mathrm{I}\right]$-iodotyrosyl) interferon- $\gamma$ in rats. The results show that microencapsulation markedly affected drug uptake and postabsorption pharmacokinetic parameters at two different drug concentrations (Table 9). Increased thyroid uptake at $4 \mathrm{~h}$ is also observed, indicating possible release of ${ }^{125} \mathrm{I}$ from interferon- $\gamma$ (Eyles et al. 1997).

Table 9. Biodistribution (\% uptake) of $\left(3-\left[{ }^{125} \mathrm{I}\right]\right.$ iodotyrosyl) interferon- $\gamma$, free and in poly(Llactide) microcapsules (MICs, $0.79 \pm 0.48 \mu \mathrm{m})$ after oral administration in rats $(\mathrm{n}=3)$.

\begin{tabular}{|l|c|c|c|c|c|c|c|c|}
\hline \multirow{2}{*}{ Tissue } & \multicolumn{3}{|c|}{15 min after Administration } & \multicolumn{3}{c|}{$4 \mathrm{~h}$ after Administration } \\
\cline { 2 - 10 } & \multicolumn{2}{|c|}{$1 \mu \mathrm{M}$ Drug } & \multicolumn{2}{c|}{$30 \mu \mathrm{M}$ Drug } & \multicolumn{2}{c|}{$1 \mu \mathrm{M}$ Drug } & \multicolumn{2}{c|}{$30 \mu \mathrm{M}$ Drug } \\
\cline { 2 - 9 } & MICs & Free & MICs & Free & MICs & Free & MICs & Free \\
\hline Portal Blood & 1.31 & 0.31 & 0.88 & 0.23 & 1.51 & 1.02 & 1.01 & 0.25 \\
\hline Systemic Blood & 8.40 & 3.32 & 6.04 & 3.46 & 5.79 & 9.23 & 7.15 & 5.92 \\
\hline Liver & 1.92 & 1.06 & 1.43 & 1.04 & 1.14 & 3.06 & 1.65 & 1.48 \\
\hline Lung & 0.88 & 0.43 & 0.81 & 0.56 & 0.91 & 1.11 & 0.88 & 1.17 \\
\hline Kidney & 1.27 & 0.52 & 0.76 & 0.43 & 1.38 & 1.16 & 0.08 & 1.05 \\
\hline Spleen & 0.21 & 0.06 & 0.22 & 0.11 & 0.14 & 0.25 & 0.20 & 0.23 \\
\hline Intestine & 25.6 & 9.5 & 12.9 & 5.2 & 13.9 & 16.3 & 7.7 & 6.4 \\
\hline Peyer's Patches & 0.10 & 0.81 & 0.21 & 0.06 & 0.07 & 0.11 & 0.20 & 0.07 \\
\hline Mesentery & 0.50 & 0.26 & 0.35 & 0.12 & 0.68 & 0.42 & 0.33 & 0.13 \\
\hline Thyroid & 0.02 & 0.27 & 0.19 & 0.13 & 1.52 & 11.4 & 4.64 & 6.90 \\
\hline
\end{tabular}

Source: Modified from Eyles et al. (1997)

${ }^{59}$ Fe-labeled magnetite particles ( $80 \mathrm{~nm}$ ) have also been used by Weissleder et al. (1989) to study biodistribution, pharmacokinetics, and toxicity. Papisov et al. (1987) investigated similar magnetic particles, but tagged them with the gamma emitter ${ }^{99 \mathrm{~m}} \mathrm{Tc}$, while Shelly et al. (1992) and Feakes et al. (1994) used liposomes containing different boron $\left({ }^{10} \mathrm{~B}\right)$ compounds. 


\section{Surface Coating}

Although several studies have focused on reducing the particle size to reduce the high uptake of particles by the liver and spleen, Gaur et al. (2000) reported a successful change in biodistribution by sterically stabilizing the particles with a layer of amphiphilic polymer chains like polyethylene glycol-phosphatidylethanolamine (PEG-PE), polyethylene oxide (PEO), poloxamers, poloxamines ${ }^{2}$, etc., on the particle surface (Allemann et al. 1993). It has been proposed that PEG-PE acts as a surface stabilizer or a hydrophilic coat. The coat decreases opsonization and subsequent uptake by the RES (Satoh et al. 1992). Gref et al. (1994) also found that the blood circulation times of particles increased as the molecular weight of covalently linked PEG increased. Five hours after injection, only one-third of the molecular weight of the PEG-coated nanospheres had been captured by the liver in comparison with uncoated particles. This phenomenon was explained by the increasing thickness of the protective PEG layer, thus preventing opsonization.

Numerous in-vitro studies show that blood components adhere strongly to hydrophobic polymer surfaces, and less strongly or not at all to hydrophilic surfaces. However, in-vivo results do not show such simple hydrophobic/hydrophilic patterns. Biodistribution of IV-injected particles indicates that relatively hydrophilic particles (e.g., proteins) are captured by the RES qualitatively to the same extent as are strongly hydrophobic particles such as polystyrene (Illum et al. 1986, 1987). This means that, although hydrophobic interactions may play a part in the capture of foreign particles by the RES, other parameters are also involved. Nevertheless, hydrophilic polymers, especially those carrying stretches of poly(ethylene glycol) (PEG), are now generally used to reduce the RES capture of both polymer nanospheres and liposomes (Figure 2) (Huang et al. 1992). A similar study by Torchilin et al. (1995) discussed the use of polyvinylpyrrolidone as a coating material for effective prolonged circulation of liposomes, broadly by reducing the extent of opsonization and consequent RES uptake.

\footnotetext{
${ }^{2}$ Poloxamers (pluronics) and poloxamines (tetronics) are a series of copolymers composed of two polyethylene glycol (PEG) blocks separated by a polypropylene glycol (PPG) block. The poloxamers and poloxamines vary in total molecular weight, PEG to PPG ratio, and surfactant properties.
} 


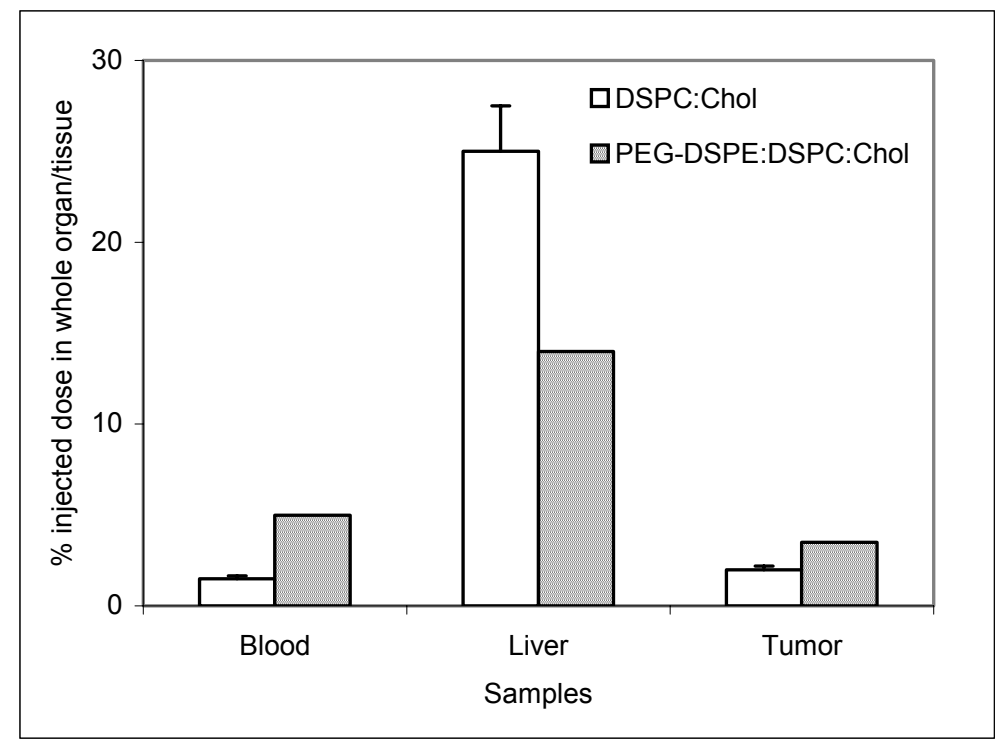

Figure 2. Distribution of Ga-loaded liposomes composed of DSPC/Chol/PEG-DSPE (2/1/0.2) or DSPC/Chol (2/1) in mice $(n=3)$ with subcutaneously implanted C-26 colon carcinoma. PEG, poly(ethylene glycol); DSPE, distearoylphosphatidylethanolamine; Chol, cholesterol; DSPE, distearoylphosphatidylcholine. Source: Modified from Huang et al. (1992).

Bazile et al. (1992) indicated that PLA microspheres coated with albumin were not found to be successful in avoiding Kupffer cell sequestration: approximately $90 \%$ of the administrated dose was found in the liver. Krause et al. (1985) also found that PLA nanoparticles accumulated predominantly in the liver after intravenous injection. Gref et al. (1994) observed that increasing the molecular weight of the PEG component from $5 \mathrm{kDa}$ to $20 \mathrm{kDa}$ increased blood circulation times while decreasing liver uptake. On comparing the blood circulating level of nanospheres composed of PLA-PEG $(\mathrm{PEG}=5 \mathrm{kDa}$ ) with that produced in the presence of poloxamine 908 ( $\mathrm{PEG}$ chain length in poloxamine $=5 \mathrm{kDa}$ ), Gref et al. observed that a blood circulating level is almost four times longer with poloxamine (12\% after $1.5 \mathrm{~h}$ for PLA-PEG and $47 \%$ after $1.5 \mathrm{~h}$ for PLA-poloxamine). However, Spenlehauer et al. (1994) have reported preliminary studies suggesting that even higher blood circulated levels can be achieved for nanoparticles containing PLA-PEG with $2 \mathrm{kDa}$ molecular weight and PLA-PEG with $5 \mathrm{kDa}$ molecular weight. They reported that after $5 \mathrm{~h}$ approximately $50 \%$ of the administrated dose of each system remains in the circulation. In the same report, the biological administration of PLA nanoparticles coated with poloxamer 188 was found to be rapidly cleared from the circulation within $30 \mathrm{~min}$ (Spenlehauer et al. 1994, Dunn et al. 1997).

\section{Surface Charge}

The adsorption of blood proteins initiates the immune system response and has been found to be related to the surface charge, where neutral or slightly negative surface charge (expressed as the zeta-potential) decreases adsorption (Grüttner et al. 1997). The zeta potential is defined as the 
potential at the shear plane between the immobilized layer (resisting shear) and bulk solution. Grüttner et al. demonstrated the proportional relation of the zeta-potential of nanoparticles at a constant $\mathrm{pH} 5$ to the basicity of the applied polymers (Figure 3). The zeta-potential of the particles and the basicity of the corresponding polymers decrease in the typical order from polyethylene imine (secondary amine) to chitosan (primary amine) further to the acid amide (polyvinylpyrrolidone) and finally to the primary alcohols starch and dextran (Grüttner et al. 1997).

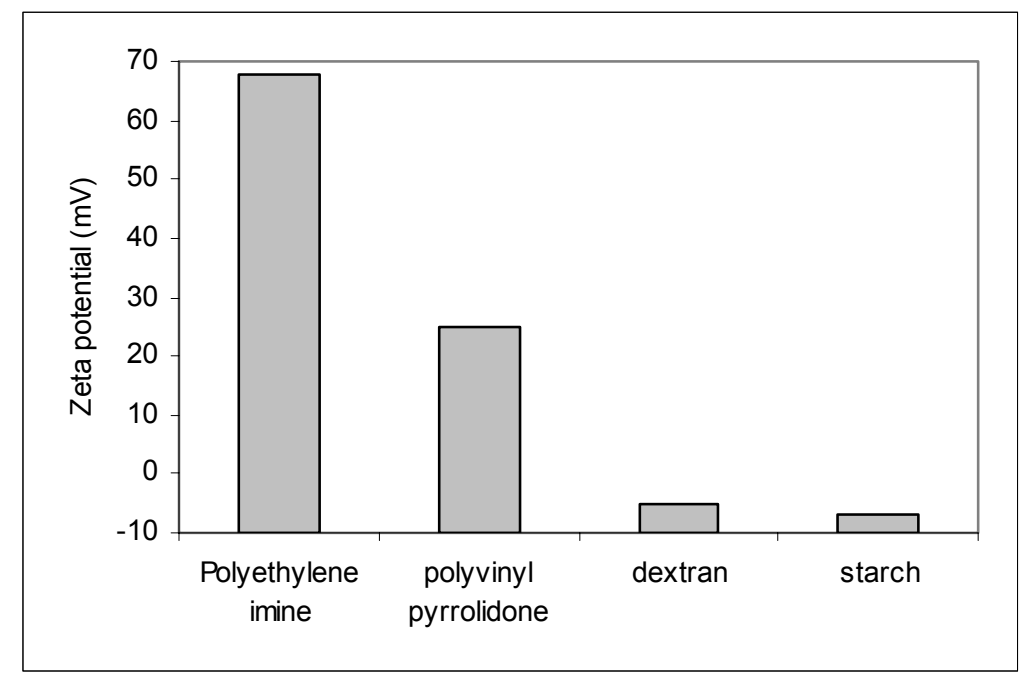

Figure 3. Zeta potential of magnetic nanoparticles as function of basicity of their polymer matrix at constant $\mathrm{pH} 5.0 \pm 0.1$. Source: Modified from Grüttner et al. (1997).

In particles modified with long PEG (generically called polyethylene oxides or PEO) chains, the shear plane, which is formed by a PEG hydrated layer on the particle surface, shifts in the direction of the solution, and as a result the zeta potential decreases. PEG chains on the particles extend from the particle surface toward the bulk water (Yoshioka 1991). Brindley et al. (1995) prepared a range of polystyrene (PS) colloids with covalently attached polyethylene oxide (PEO) $(\mathrm{PEG}=2 \mathrm{kDa}$, or PEO2000) chains. The zeta potentials of the dialyzed PS and PS-PEO2000 colloids, as a function of $\mathrm{pH}$, are displayed in Figure 4. While an increase in $\mathrm{pH}$ gives rise to very little change in zeta potential, a significant difference in the $\mathrm{ZP}-\mathrm{pH}$ profile was obtained for the copolymer colloids (Brindley et al. 1995). 


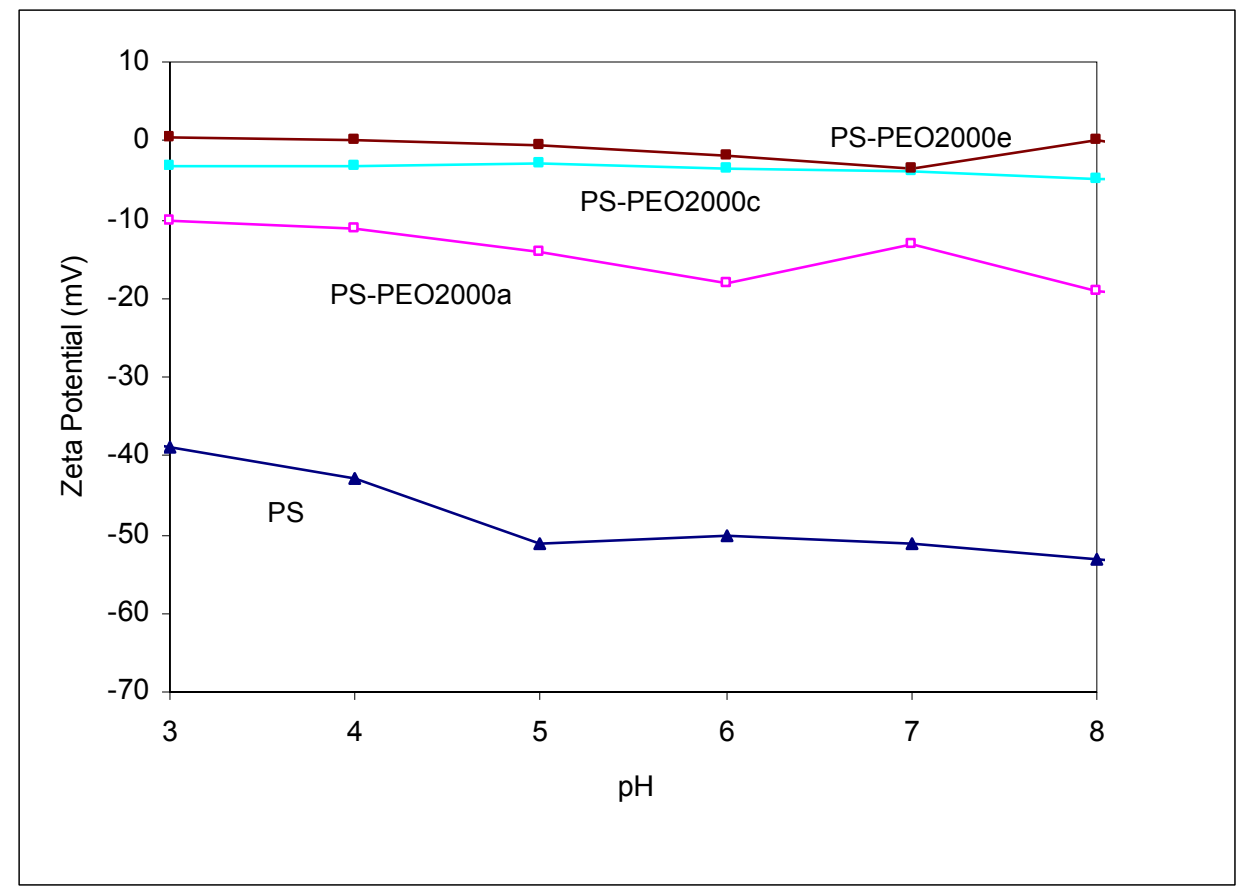

Figure 4. Zeta potential of PS and PS-PEG2000 colloids in $0.001 \mathrm{M}$ ionic strength buffer with changing $\mathrm{pH}$. Source: Modified from Brindley et al. (1995).

Gomez-Lopera et al. (2001) also compared the surface properties of PLA and PLA-magnetite spheres to those of the magnetite composite. Magnetite particles show a distinct isoelectric point (or $\mathrm{pH}$ of zero zeta-potential) in the vicinity of $\mathrm{pH} 7$ (Figure 5), in agreement with previous determinations from other authors (Regazzoni et al. 1983). In contrast, PLA microspheres always bear a net negative surface charge, and the data suggest an isoelectric point below pH 2 . If an efficient coverage of magnetite by PLA is achieved, it is reasonable to predict that composite particles will have an isoelectric point lower than $\mathrm{pH} \mathrm{7,} \mathrm{and} \mathrm{also} \mathrm{that} \mathrm{pH}$ should have a lesser effect on zeta potential than it has in the case of magnetite (Gomez-Lopera et al. 2001). The electrokinetic properties of composite particles do not coincide with those of pure PLA, as one would predict in the case of an optimum coverage. A rather intermediate electrokinetic behavior is found. This can be explained simply by assuming that the polymer shell is not homogeneous, or that some magnetic particles remain partially uncovered. Nevertheless, the presence of a layer of PLA is clear, and modifies significantly the surface electric properties of magnetite. 


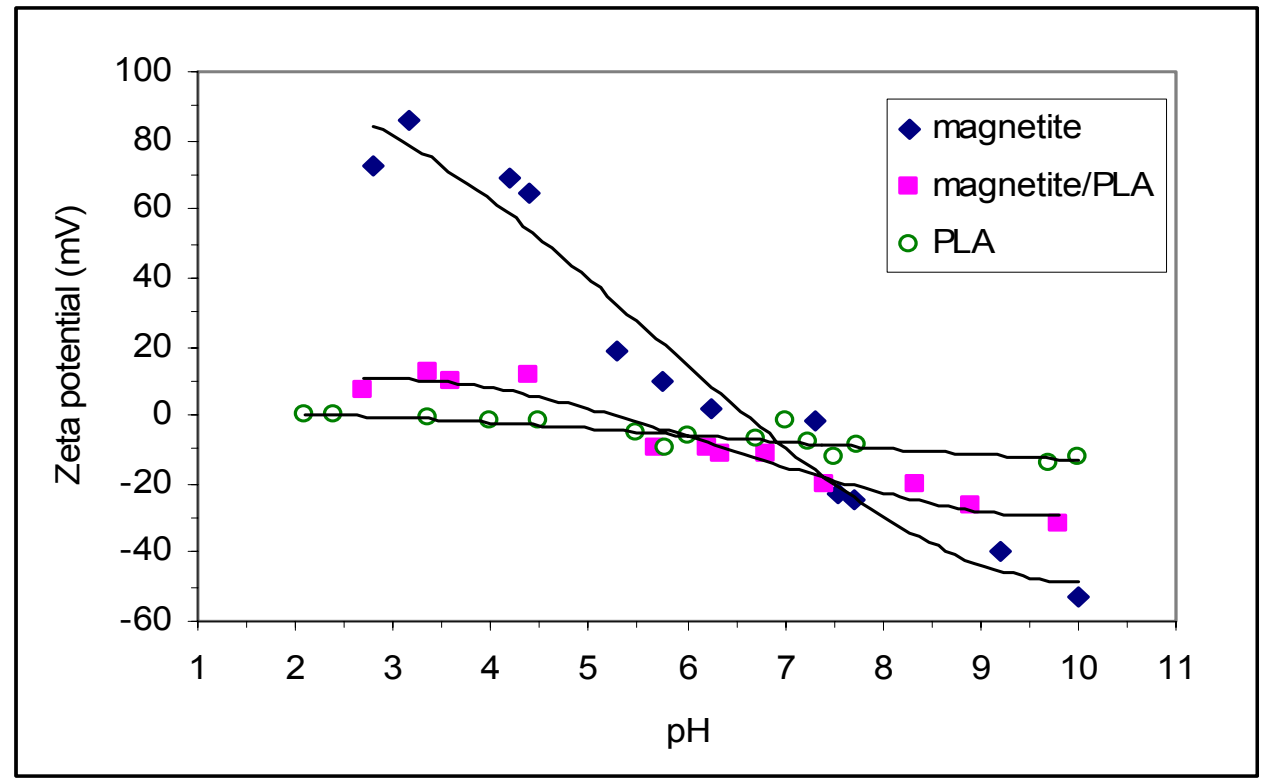

Figure 5. Zeta potential of magnetite, PLA, and mixed particles as function of $\mathrm{pH}$ in presence of $10^{-3} \mathrm{M} \mathrm{KNO}_{3}$. Source: Adapted from Gomez-Lopera et al. (2001).

Other water-soluble polymers have also been investigated for prolonging particle circulation time in the bloodstream. Torchilin et al. (1994) used computer simulation to show that a relatively small number of surface grafted hydrophilic flexible polymer chains can create a dense protective conformational layer over the particle surface, preventing the access of plasma proteins. A rigid polymer fails to form such a dense protective layer even when the polymer is hydrophilic, such as dextran. Particles modified with polyacrylamide or polyvinylpyrrolidone showed similar good protective results (Arshady and Monshipour 1999).

\section{POL YETHYLENE GLYCOL}

Covalent attachment of biologically active compounds to polymers and polymeric spheres became one of the methods for alteration and control of biodistribution, pharmacokinetics, and, often, toxicity of these compounds (Duncan and Kopecek 1984). As discussed in the preceding section, one of the most popular polymeric materials used for this purpose is polyethylene glycol (PEG). It possesses an ideal array of properties: excellent solubility in aqueous solutions (Pang 1993), extremely low immunogenicity and antigenicity (Dreborg and Akerblom 1990), and has the advantage of being non-toxic and was approved by the Food and Drug Administration for internal use in humans (Harris 1985).

Jeon and Andrade proposed a mathematical model, taking into account the four types of interactions between a protein and hydrophobic substrate (Figure 6). They stated that the best conditions for protein repulsions were found to be long PEG chain length and high surface density. As shown in the Figure 6, if $\mathrm{D}$ is the distance between the anchorage to the substrate of the two terminally attached PEG chains, in the case of small proteins (approximately $4 \mathrm{~nm}$ in diameter), D should be around $1 \mathrm{~nm}$, whereas for larger proteins $(6-8 \mathrm{~nm})$, D should be around $1.5 \mathrm{~nm}$ (Jeon and Andrade 1991). 


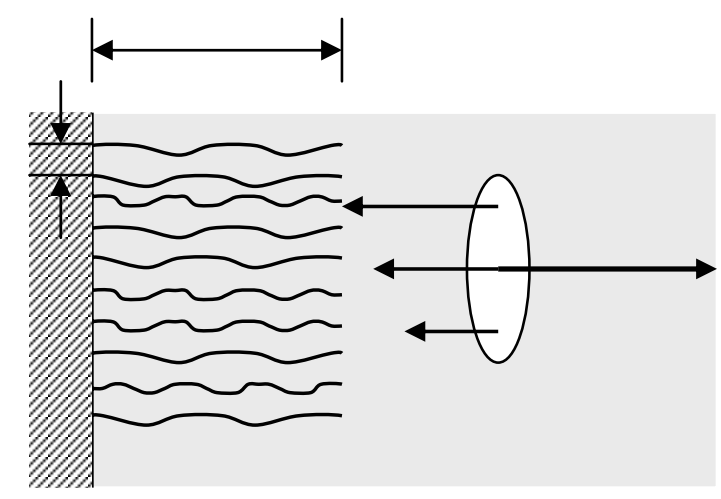

Figure 6. Interactions between protein and hydrophobic substrate with attached PEG chains (conceptual drawing adapted from Gref et al. 1995). $\mathrm{HA}_{\mathrm{P}-\mathrm{S}}=$ Hydrophobic attraction between the protein and substrate; $\mathrm{SR}_{\mathrm{PEG}}$ $=$ steric repulsion resulting from PEG chain constriction; $\mathrm{VDW}_{\mathrm{P}-\mathrm{S}}=\mathrm{van}$ der Waals attraction between the protein and the substrate; $\mathrm{VDW}_{\mathrm{P}-\mathrm{PEG}}=$ van der Waals attraction between the protein and the PEG chains.

Since polyethylene glycol has two equivalent hydroxyl groups, it could act as a potential crosslinking agent for any system to which it is attached. One very important derivative used in a number of derivatization reactions, which has one hydroxyl group blocked, is the monomethoxypolyethylene-glycol (MPEG). It is generally used when multiple chains of polymers have to be linked or conjugated to the intended bioactive substrates. Because it is structurally simple and has only one derivatizable end group, the use of MPEG minimizes cross-linking possibilities and leads to improved homogeneity of the conjugate. Thus, it is usually a starting material of choice for the covalent modification of proteins, biomaterials, particulates, lipids, drugs, etc., as shown in the following equation (Bhadra et al. 2002).

$$
\mathrm{MeOH}+\mathrm{H}\left(\mathrm{OCH}_{2} \mathrm{CH}_{2}\right)_{n} \mathrm{OH} \rightarrow \mathrm{MeO}\left(\mathrm{CH}_{2} \mathrm{CH}_{2} \mathrm{O}\right)_{n} \mathrm{H}+\mathrm{H}_{2} \mathrm{O}
$$

Although most of the following examples are focused on the conjugation of PEG to nanoparticles and microparticles, many of the chemical methods used for particle PEGylation are relevant regardless of the end use of a particular conjugate.

As one would expect, PEG-coated particles have PEG chains attached to their surface at one chain end, in a brush configuration, which should avoid or reduce the interactions with blood proteins and therefore impart RES-avoiding properties. To achieve the core-shell structure, Gref et al. (1995) illustrated a synthesis for block amphiphilic polymers of the type PEG-R. "R" is chosen from among the bioerodible polymers (e.g., PLA, PLGA). The two blocks have a tendency to easily phase-separate in the presence of water (Shah et al. 1994) and have different solubilities in water and organic solvents. This phase separation is used to obtain the core-shell structure by an oil-in-water $(\mathrm{O} / \mathrm{W})$ emulsification procedure (Figure 7). For this, PEG-R polymers are dissolved in an organic solvent immiscible with water (such as ethyl acetate or methylene chloride). The o/w emulsion is formed in an aqueous phase, and the organic solvent is allowed to slowly evaporate. This leads to a progressive increase in polymer concentration 
inside the droplets. $\mathrm{R}$ is insoluble in water, but highly soluble in the organic solvent; conversely, PEG is highly water-soluble, soluble in methylene chloride, and practically insoluble in ethyl acetate. This leads to a tendency of PEG chains to migrate towards the water phase to form sterically stabilized particles (Figure 6) with the core presumably mostly composed of R-chains. After complete solvent evaporation, the particle core solidifies, thus entrapping the hydrophobic biologically active molecules (Gref et al. 1995).

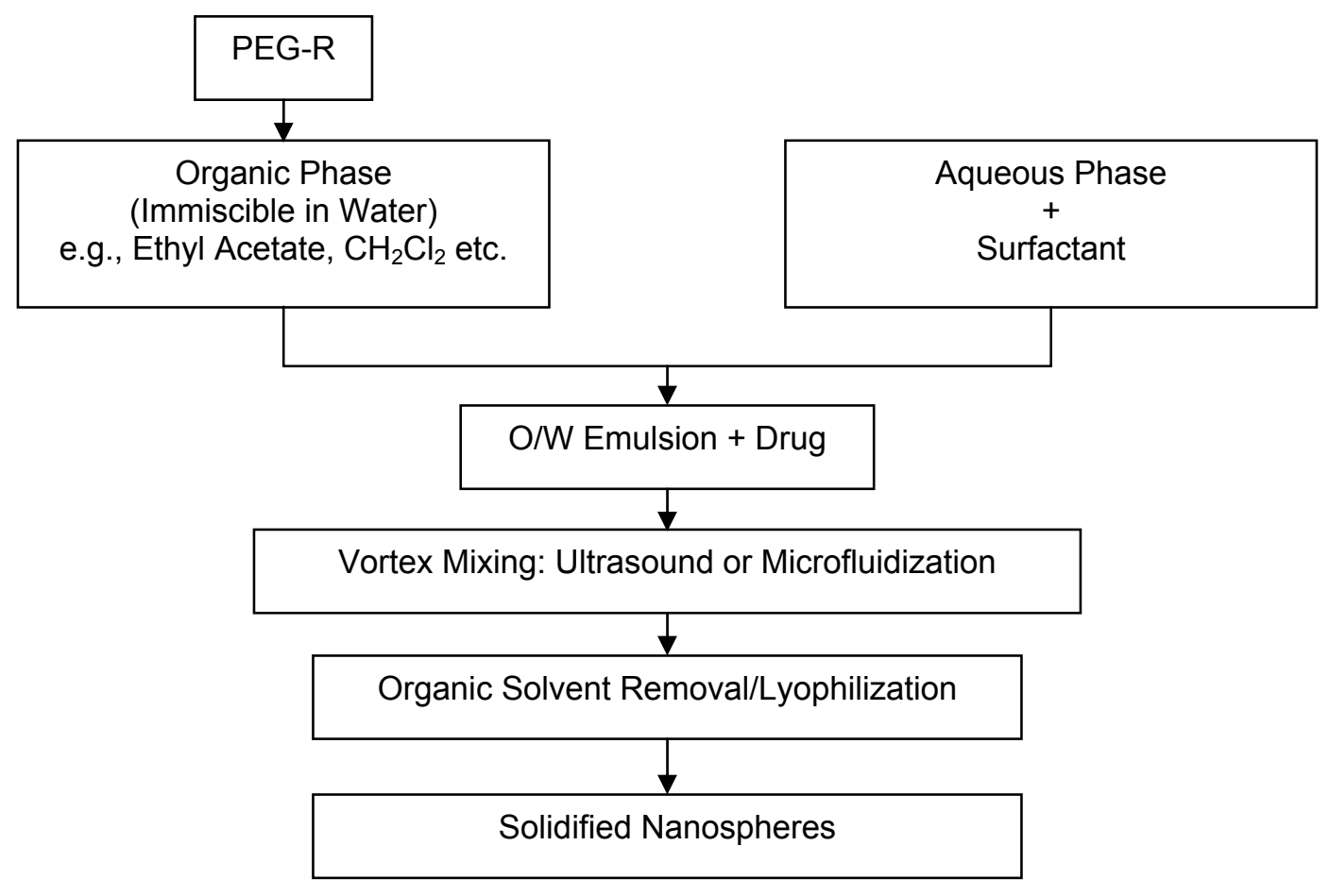

Figure 7. Schematic representation of particle fabrication following an emulsionsolvent evaporation procedure. Source: Modified from Shah et al. (1994).

Efimov et al. also described a solid-phase method of general applicability for preparation of 3'-, 5'- and 3',5'-PEG-oligonucleotides. It is based on attachment of mono(dimethoxytrityl)-PEG to porous beads (controlled-pore glass) through a phthalate ester residue, followed by removal of the DMT group, assembly of the desired oligonucleotide sequence by the phosphoramidite method of solid-phase oligonucleotide synthesis, and then cleavage of the 3'-PEG conjugate by aminolysis. A phosphoramidite derivative of PEG was used to obtain 5'- or 3',5'-conjugates in the last step, prior to cleavage from the support (Efimov et al. 1993).

Finally, a convenient method for grafting an MPEG chain onto dextrans was recently described by Hoste et al. (1994). It involves activation of some of the sec-hydroxy groups of the polysaccharide with p-nitroxy groups of the polysaccharide with p-nitrophenyl chloroformate followed by aminolysis with MPEG-NH $\mathrm{N}_{2}$. The extent of MPEG grafting is controllable by the amount of the reactive p-nitrophenyl carbonate groups on dextran. The method appears to be generally applicable to polysaccharides. 


\section{METHODS FOR SYNTHESIS OF PARTICLES COMPOSED OF POLY(LACTIC ACID) AND POLY(LACTIC-CO-GLYCOLIC ACID)}

Many types of polymers have been investigated for in-vivo applications. Our approach to developing a particle carrier for delivering drugs is to focus on poly(lactic acid) and poly(lacticco-glycolic acid) macromonomers. There is a plethora of literature describing synthesis methods and drug encapsulation, release kinetics, biostability, and in-vivo properties such as macrophage engulfment, biodegradation, and organ disposition. In this section we describe specific synthesis procedures for PLA or PLGA being considered for our programs in chemotherapeutic, radiotherapeutic, protein, and enzymatic drug delivery. Following the written summary, Table 10 summarizes the critical synthesis factors. From this summary, we can select one or several methods for follow-up investigation.

\section{a. Hydrogels}

PEG-PMA

Donini et al. (2002) used hydrogels of polyethylene glycols grafted on poly(methacrylic acid) (PMAA). The PEG molecular weights were 200, 400, and 1000 Da in PEG-monomethylether monomethacrylate (PEGMA). Solution precipitation polymerization was used. The two monomers of methacrylic acid (MAA) and PEGMA were mixed in 1:1 molar ratio MAA to ethylene glycol (EG) in water. Tetraethylene glycol dimethacrylate cross-linker was added to $0.75 \%$ moles of the total moles of the monomer. The photo-initiator 1-hydroxycyclohexyl phenyl ketone was added at $0.5 \% \mathrm{w} / \mathrm{w}$ of the monomer mixture. After dissolution of components, the solution was diluted in deionized water and purged for $20 \mathrm{~min}$ to remove dissolved oxygen. The flask was exposed to UV light for $15 \mathrm{~min}\left(100 \mathrm{~mW} / \mathrm{cm}^{2}\right)$. A milky white solution of nanospheres was produced. Then the nanospheres were washed in 25,000 and 300,000 molecular weight (MW) dialysis membranes. Cryoprotectants were added to the nanosphere solution prior to freeze-drying.

Dynamic light scattering showed that the polydispersity index was larger for unwashed nanospheres, with an average size of $300 \mathrm{~nm}$. After washing, the polydispersity index was reduced significantly, with an average size of $300 \mathrm{~nm}$. The dispersion $\mathrm{pH}$ was $\sim 3$. No effect on the polydispersity index was seen when $25,000 \mathrm{MW}$ or $300,000 \mathrm{MW}$ dialysis tubing was used. When the PEG chain length was varied, the lowest PEG chain produced the largest particle $(300 \mathrm{~nm})$ and largest polydispersity index $(\mathrm{PI}=0.4)$ while the highest PEG chain produced $<20 \mathrm{~nm}$ nanoparticles and $\mathrm{PI}<0.05$. After freeze-drying, the particles were resuspended in acidic or alkaline media. In an acidic suspension $(\mathrm{pH} 3)$ the particles agglomerated, but in an alkaline suspension, they resuspended. Donini et al. (2002) tested various cryoprotectants (also called excipients): Trehalose, glucose in 1-5\% w/w and PEG 3400, PEG8000, PVP10000, PVP40000, Pluronic P123, Pluronic F68 at $0.1-2 \%(w / w)$. Samples of the dispersion were added to $\mathrm{NaOH}$ $(0.1 \mathrm{M})$ to swell the hydrogels. When the particle size reached $500-600 \mathrm{~nm}$, the cryprotectant was added. Then the $\mathrm{pH}$ was lowered to 3 with $\mathrm{HCl}$.

Only Pluronic (PO-EO) effectively countered agglomeration. However, Pluronic P123 also increased particle size and the polydispersity index. Pluronic F68 at $0.5 \%$ was best and performed better than P123 because it did not increase particle size. When the samples were 
freeze-dried with liquid $\mathrm{N}_{2}$, the nanoparticles resuspended better than by conventional freezing, although sonication resuspended samples after conventional freezing.

Arias et al. (2001) chose a member of the polyalkylcyanocrylate family because of its high reactivity, ability to polymerize in water, biodegradability, and drug compatibility. Arias et al. cite four references that document drug encapsulation studies. The polyalkylcyanocrylates degrade more rapidly than poly(lactic acids) but their degradation products may be slightly more toxic. They crystallized magnetite by precipitation of ferrous salt in sulfate solution in the absence of oxygen. The polymeric particles were prepared following the method of Couvreur et al. (1979). The monomer $(1 \% \mathrm{w} / \mathrm{v})$ was added dropwise to $50 \mathrm{~mL}$ of aqueous polymerization media $(2 \mathrm{mM} \mathrm{HCl})$ stirred at $1,000 \mathrm{rpm}$. Magnetite was added to the polymerization medium to $0.75 \% \mathrm{w} / \mathrm{v}$ and in the range of $1: 4$ and $4: 1$ monomer/magnetite proportions. After $3 \mathrm{~h}$ the medium was neutralized by addition of $1 \mathrm{~mL}$ of $0.1 \mathrm{M} \mathrm{KOH}$. The suspension was cleaned by ultracentrifugation at $20,000 \mathrm{rpm}$. The particles were dried at $35^{\circ} \mathrm{C}$ in a vacuum oven and stored.

The magnetite nanoparticles were $82 \pm 24 \mathrm{~nm}$ while the non-magnetic polymeric nanoparticles were $380 \pm 120 \mathrm{~nm}$. The best monomer:magnetite ratio was $4: 3$, producing particles that were $144 \pm 15 \mathrm{~nm}$ where the magnetite is covered by a polymer shell of approximately $30 \mathrm{~nm}$. When the monomer ratio was lower, a very thin polymer coating was observed, but at times it did not completely cover the magnetite. When the monomer was excessive then polymer sheets were formed, reducing dispersibility. When the 4:3 mixture was used, the electrophoretic mobility of the resulting composite particles was identical to that of the non-magnetic polymer particles $(-40 \mathrm{mV}$ at $\mathrm{pH}$ 7). Incubation in water shows that about $300 \mathrm{~h}$ are required for the zeta potential to reach the values of bare magnetite, indicating polymer degradation. Thermodynamic calculations show that the free energy for interaction of the magnetite and polymer are negative, indicating that the polymer prefers to remain with the magnetite rather than isolated in the water phase.

A paper by Morita et al. (2000a) describes in detail the first step in the solid-in-oil-in-water technique. They used PEG 2,000-70,000 to form a primary solid microparticle containing bovine serum albumin as the model protein. The procedure called for adding protein and PEG to water and freeze-drying. The particles were analyzed after resuspending in methylene chloride.

The PEG/protein ratio was varied (4 mg protein with 0-36 mg PEG 6,000); the larger the ratio the smaller the microparticles: $0.3-3 \mu \mathrm{m}$ at $9 / 1$ and $10-100 \mu \mathrm{m}$ at $1 / 1$ with a definite inflection point signaling the critical point during condensation. Longer-chain PEGs were tested to 70,000 MW and showed no influence on size for PEG $>4,000$ MW. For PEG 4,000 and 2,000 even higher ratios were best to keep microparticles smaller than a few micrometers (exact values were not given). During encapsulation Morita et al. (2000a) found that no protein activity was lost, and a PEG/protein ratio needed to make microparticles smaller than a few micrometers was higher for smaller molecular-weight proteins. They determined a stable PEG/protein ratio to achieve proper phase separation and no particle agglomeration-PEG at $6-8 \%$ and protein $0-10 \%$, with a critical ratio of 0.22 . 


\section{b. Oil-in-Water \\ $\underline{\text { PLGA }}$}

PLGA in methylene chloride at $500 \mathrm{mg} / \mathrm{mL}$ was emulsified with $0.25 \mathrm{~mL}$ of $0.5 \% \mathrm{w} / \mathrm{v}$ poly(Llysine) hydrobromide (pLys) surface-active functional polymer (150-300 kDa) solution at various degrees of dissociation or $0.5 \%$ PVA in water and vortex-mixed (Cui and Schwendeman 2001). The emulsified PLGA was then hardened in $60 \mathrm{~mL}$ deionized water for $3 \mathrm{~h}$ under stirring, collected by centrifugation, washed with $\mathrm{NaCl}$, suspended in sucrose and $\mathrm{NaCl}$, freeze-dried with liquid $\mathrm{N}_{2}$, and lyophilized. The sucrose was removed from lyophilized particles by washing with PBS containing Tween- 80 and was centrifuged. The particle sizes were all very large ( $>$ few micrometers) and thus not very useful for our work.

\section{$\underline{\text { PLA }}$}

Mosquiera et al. (2000) used the method of Fessi (1989). PLA (9-251 kDa) was used with and without Poloxamer 188, and $60 \mathrm{mg}$ of total polymer was solubilized in $2 \mathrm{~mL}$ acetone and added to $8 \mathrm{~mL}$ acetone containing lipophilic surfactant and $250 \mu \mathrm{L}$ of oil (oils included soybean, ethyl oleate, and Miglyol). This organic solution was added to $20 \mathrm{~mL}$ of water under moderate agitation with and without $0.375 \%$ Poloxamer 188 . The solvent was evaporated to $10 \mathrm{~mL}$ under reduced pressure. No other measures were taken to purify the sample.

Mosquiera et al. argue that the resulting particle size increases with increasing interfacial tension of oil in water and oil viscosity. As the PLA molecular weight increases, the surface charge decreases due to fewer end member carboxyl groups in the long chains. Lecithin (as Epikuron 170) was added as a secondary functional polymer and was found to influence the resulting surface charge. As surfactant is increased, particle size decreases until Epikuron 170 is 30 $\mathrm{mg} / \mathrm{mL}$ and not affected thereafter; the zeta potential became less negative although zeta potentials for all formulations were very negative $(<-20 \mathrm{mV} @ \mathrm{pH} 7)$. The nanocapsules had densities of $1.015-1.052 \mathrm{~g} / \mathrm{cm}^{3}$.

\section{PLA/PLGA}

McGee et al. (1995) used a modified procedure that they claim leads to fewer aggregated particles. They used Resomer RG503 (90kDa, 50:50 lactide:glycolide), ResomerRG858 (50kDa, lactide:glycolide 85:15), and Resomer R208 (300kDa). They dispersed $10 \mathrm{mg}$ oval albumin as a model protein in $300 \mathrm{~mL}$ of water and dispersed the resulting mixture into $30 \mathrm{~g}$ of silicone oil kept at $5-15^{\circ} \mathrm{C}$ for $4 \mathrm{~min}$. A $2 \%(\mathrm{w} / \mathrm{v})$ solution of RG503 in dichloromethane was added by syringe and homogenized for $5 \mathrm{~min}$. The microparticles that were formed were transferred to $300 \mathrm{~mL}$ of heptane and stirred for $1 \mathrm{~h}$ and washed with heptane.

They investigated, to some extent, many variables, including volume of dichloromethane, use and quantity of surfactants, and temperature of emulsion solution. For RG503 (90Kda) PLGA the spheres were broadly distributed, about 5-10 $\mu \mathrm{m}$.

\section{PLGA-PEG}

Gref et al. (1994) describe the use of PEG (5,000 MW), PLGA (45,000 MW) at a lacticacid:glycolic acid ratio $=75: 25$. PLGA-PEG was prepared by direct reaction of terminal amine groups from the monamine, monomethoxy PEG with PLGA. Co-block polymers were also synthesized (for in-vivo work) by ring-opening polymerization at $114^{\circ} \mathrm{C}$ of lactide and glycolide 
in the presence of monomethoxyPEG (36:9:5 molar ratio) using stannous octoate as the catalyst (0.2 wt \%). The lactic acid:glycolic acid ratio was 3:1 in each case and PEG was $10 \%$ by weight (PEG MW varied from 5 to $20 \mathrm{kDa}$ ). Solvent evaporation was used (modified from Beck et al. 1986). Specifically, the copolymer was dissolved in solvent (ethyl acetate or methylene chloride, $25 \mathrm{mg} / 2 \mathrm{~mL}$ ). This was poured into $30 \mathrm{~mL}$ of deionized water and an oil-in-water emulsion was formed by vortexing ( $30 \mathrm{~s}$ ) and sonicating ( $1 \mathrm{~min}, 40 \mathrm{~W}$ output). The organic solvent was slowly removed by evaporation and gentle stirring at room temperature for $2 \mathrm{~h}$.

The particles were monomodal in size at $140 \mathrm{~nm}$. Distribution in mice showed that higher molecular weight PEG led to longer circulation, with 20\% left in circulation at $1.5 \mathrm{~h}$ and $20 \mathrm{kDa}$ PEG.

Dunn et al. (1997) used PLGA (75:25, 63 kDa, ResomerRG755), commercially available polystyrene nanoparticles (172 nm), Poloxamer 407 (11,500 MW), Poloxamine 904 (6700 MW), and Poloxamine 908 (25,000 MW). The PLGA nanospheres were prepared using the technique of Fessi (1989), an oil-in-water technique. The PLGA particles were prepared both in the presence of surfactant and without surfactant. The particles prepared without surfactant were subsequently incubated in surfactant solution to create a sorbed hydrophilic layer. PLGA was dissolved in $5 \mathrm{~mL}$ of acetone to make a $0.5 \%$ or a $0.25 \% \mathrm{w} / \mathrm{v}$ solution. This was added dropwise into a water solution, either containing or not containing surfactant (the Poloxamer/Poloxamines listed above) with mixing at ambient temperature. The resultant nanoparticles were passed through a $1-\mu \mathrm{m}$ filter after the volatile solvent had evaporated. Polystyrene nanoparticles $(0.3 \%$ $\mathrm{w} / \mathrm{v})$ were incubated in surfactant $(2 \% \mathrm{w} / \mathrm{v})$ for $24 \mathrm{~h}$ at room temperature for comparison.

The PLGA nanoparticle size was 99-122 nm, depending on the surfactant copolymer used, with a very small standard deviation $(<1 \mathrm{~nm})$. However, the zeta potential was -15 to $-27 \mathrm{mV}$, which is strongly negative for in-vivo applications. This range was not significantly different than the range determined for bare PLGA microspheres incubated in surfactant Poloxamer/amine solution. The zeta potential did not change after radiolabeling. Also, the zeta potential for incubated PLGA microspheres was not different than that of incubated polystyrene microspheres $(-14.3 \pm 0.2)$. The uptake of non-parenchymal liver cells was reduced by a factor of 10 for polystyrene particles coated with the surfactant, while the uptake onto PLGA spheres was reduced by a factor of 2-3. The PLGA nanoparticles synthesized in the presence of surfactant and those incubated in surfactant performed similarly (1.4-2.2\% uptake in rat serum). In vivo studies in rats (150-g rats, $1 \mathrm{mg}$ injected) showed that polystyrene nanoparticle removal is immediate when Poloxamer/amine is not present (about 15\% remaining after $15 \mathrm{~min}$ ) but prolonged when the surfactant is present $\left(\mathrm{T}_{1 / 2}=3 \mathrm{~h}, 45 \%\right.$ remaining on PS-PEO $\left.11.5 \mathrm{kDa}\right)$. Bare PLGA spheres (12\% remaining after $5 \mathrm{~min}$ ) survived similarly to the bare polystyrene. The PLGA coated with surfactant survived equally as well $\left(\mathrm{T}_{1 / 2}=2.5 \mathrm{~h}, \mathrm{PEO}=11.5 \mathrm{kDa}\right)$ as the polystyrene-surfactant particles. No significant difference was seen when the particles were PEGylated during synthesis or by incubation, or when the incubated particles were cleaned of excess surfactant (by dispersal in water prior to injection) or in excess surfactant (by dispersal in $1 \% \mathrm{w} / \mathrm{v}$ surfactant solution). Clearly the Poloxamer $407(11.5 \mathrm{kDa})$ produced the best results. With these particles, about $20-30 \%$ of the particles were in the lung, $25 \%$ in the spleen, and 43 $50 \%$ in the blood after $3 \mathrm{~h}$. 


\section{rac-PLA-PEG}

Mosqueira et al. (2001) cited the work of Fessi (1989) for the solvent evaporation method. Poly(rac-lactic acid) of 42,000 MW was used with Poloxamer 188 to make non-surface-modified spheres (details not provided in paper). The diblock poly(rac-lactic acid)-PEG polymer was added to PLA with a PEG content of $10-30 \% \mathrm{w} / \mathrm{w}$ of PEG (with respect to total polymer) in acetone, with lecithin and Miglyol 810N (no values given). They dissolved $6 \mathrm{mg}$ of polymer in 2 $\mathrm{mL}$ acetone containing $7.5 \mathrm{mg}$ soy lecithin and $25 \mu \mathrm{L}$ of Miglyol. This was poured into $4 \mathrm{~mL}$ of water $(0.375 \% \mathrm{w} / \mathrm{v}$ Synperonic F68 added for non-surface- modified spheres). The particles were filtered through a $0.45-\mu \mathrm{m}$ filter. Dynamic light scattering was used to determine size and they studied in vitro properties and stability and biodistribution in $25-\mathrm{g}$ mice.

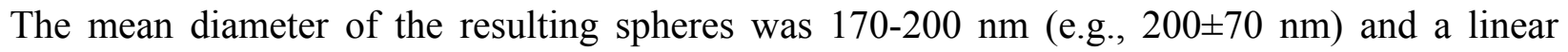
dependence on size with PEG content (10-30\%) and polydispersity index, $\mathrm{PI}=0.1-0.3$. The zeta potential was still very negative even at 30\% PEG $20 \mathrm{kDa}(-38 \mathrm{mV})$. A PLA-PEG of 45-20 kDa nanoparticles showed that 30\% remained after $1 \mathrm{~h}$ in the blood plasma and $6 \%$ remained in the liver. The authors state that using more than one PEG chain per PLA chain in the copolymer might help reduce distance between PEG on surface and eliminate the gaps that allow for opsonization.

\section{c. Solid-in-Oil-in-Water}

\section{PLA/PLGA-PEG}

Morita et al. (2000b) used PEG 6 kDa and 70 kDa, PLA $20 \mathrm{kDa}$, and PLGA (50:50) $10 \mathrm{kDa}$ and $20 \mathrm{kDa}$. The method was solid-in-oil-in-water, where the protein was micronized in PEG solution (Morita-2000a). Protein (4 mg) and 0-28 mg of PEG6K was dispersed in $1 \mathrm{~mL}$ of water and freeze-dried. This was dispersed in $1 \mathrm{~mL}$ of methylene chloride and the size distribution studied. Water $(1 \mathrm{~mL})$ containing $12.5 \mathrm{mg}$ protein and PEG6K or PEG70K was lyophilized as above and used for protein-loaded microspheres. The PLA or PLGA was weighed in a tube containing the lyophilized protein-PEG mixture. The total amount of solids (PLA or PLGA+PEG+protein) was $500 \mathrm{mg}$. To dissolve the solids, $1350 \mathrm{mg}$ of methylene chloride was added. The resultant was added to $4 \mathrm{~mL}$ of $0.25 \% \mathrm{w} / \mathrm{v}$ methylcellulose solution at $15^{\circ} \mathrm{C}$ and homogenized for $4 \mathrm{~min}$ at $8,000 \mathrm{rpm}$ and quickly poured into $400 \mathrm{~mL}$ of deionized water. The temperature was raised to $30^{\circ} \mathrm{C}$ for $3 \mathrm{~h}$ while mixing vigorously by propeller at $400 \mathrm{rpm}$. The resultant was filtered through a $20-\mu \mathrm{m}$ filter, washed with deionized water, and freeze-dried. They also used a w/o/w emulsion where $0.2 \mathrm{~mL}$ of water containing $12.5 \mathrm{mg} \mathrm{HRP}$ was homogenized in $1 \mathrm{~mL}$ of polymer solution for $2 \mathrm{~min}$ at $8,000 \mathrm{rpm}$. The resulting mixture was immediately emulsified in $4 \mathrm{~mL}$ of $0.25 \%$ methylcellulose and treated as described above.

The results showed that the PEG:protein ratio in the micronization step must be $>5$ to create microparticles less than a few micrometers in diameter. Rapid freezing was not done because of fear of protein denaturization. The size of the final microparticles was 35-40 $\mu \mathrm{m}$, although microparticles of about $100 \mu \mathrm{m}$ could be produced depending on conditions. Increasing the quantity of PEG used in the micronization method with the protein proved to re-encapsulate more protein (entrapment efficiency). The authors describe another method called polymer alloying in which long-chain PLA and short-chain PLGA are mixed. The PLGA phase-separates and has a higher affinity for protein, so the PLGA will be drug-loaded in a matrix of PLA. This 
may reduce the "initial burst" phenomena associated with immediate dissolution of protein at the oil/water surface. Three separate formulations of the polymer alloy method were used and data showed that the release kinetics can be modified such that a slow constant release of protein occurs (Morita et al. 2000b).

\section{d. Water-in-Oil-in-Water PLGA}

The PLGA (ResomerRG503, 9kDa 50:50) was dissolved in dichloromethane to $6 \% \mathrm{w} / \mathrm{v}$ and this was homogenized with $6 \%$ antigen (ovalbumin) in water. This emulsion was added to a much larger volume of 5\% PVA solution and homogenized. The typical antigen concentration was $1 \% \mathrm{w} / \mathrm{v}$ and particle size was $5.34 \mu \mathrm{m}$ as determined by laser diffractometry.

The purpose of this work was to develop a biodegradable microparticle to deliver controlled amounts of antigen following phagocytosis. Each rat was injected with $10 \mathrm{mg}$ of microparticles. The results show that antigen levels were sustained for 12 weeks (O’Hagan et al. 1991).

The double emulsion technique is often used to encapsulate protein. Here, the aqueous protein solution was sonicated in an organic solution to form a water-in-oil (w/o) emulsion. Then this was added to an aqueous solution and homogenized. Harsh conditions can denature the protein, however. Fourier transform infrared spectroscopy (FTIR) following bovine serum albumin and chicken egg white lysozyme in PLGA microspheres showed one-third lower activity of protein. Adding stabilizing trehalose may help, they note.

O'Hagan et al. used PLGA $(50: 50,25 \mathrm{kDa})$ in PVA $(25 \mathrm{kDa})$. To $200 \mu \mathrm{L}$ of protein solution $(100 \mu \mathrm{L}$ of $100 \mathrm{mg} / \mathrm{mL}$ protein at $\mathrm{pH} 7.4$ and $100 \mu \mathrm{L}$ of water or trehalose solution), they added $1 \mathrm{~mL}$ of $200 \mathrm{mg} / \mathrm{mL}$ PLGA in methylene chloride, and sonicated with a probe. They added this primary emulsion to $100 \mathrm{~mL} \mathrm{1 \%} \mathrm{PVA} \mathrm{in} \mathrm{water} \mathrm{and} \mathrm{homogenized} \mathrm{at} \mathrm{3,000} \mathrm{rpm} \mathrm{for} 1 \mathrm{~min}$, then stirred for $3 \mathrm{~h}$. Finally, the emulsion was centrifuged, washed three times with water, freezedried, and stored under dessicant at $-20^{\circ} \mathrm{C}$. No particle size data are given. Stabilizer (trehalose) helped a little by increasing serum albumin activity from 71 to $74 \%$ (Fu et al. 1999).

\section{$\underline{\text { PLA }}$}

Gomez-Lopera et al. (2001) synthesized magnetite by mixing two solutions flushed with $\mathrm{N}_{2}$, these being $0.14 \mathrm{M} \mathrm{KOH} / 0.23 \mathrm{M} \mathrm{KNO}_{3}$, and $1: 196 \% \mathrm{H}_{2} \mathrm{SO}_{4}$ in water with $55.6 \mathrm{~g} \mathrm{FeSO}_{4} \cdot 7 \mathrm{H}_{2} \mathrm{O}$ and $200 \mathrm{~mL}$ of water. Base solution $(1225 \mathrm{~mL})$ was mixed with $88.8 \mathrm{~mL}$ of the iron acid solution, forming a gel. This was maintained at $90^{\circ} \mathrm{C}$ for $4 \mathrm{~h}$ and then magnetically separated from 35 cycles of wash solution and dried at $60-70^{\circ} \mathrm{C}$ in a vacuum oven. They used a w/o/w method in which $20 \mathrm{~mL}$ of water was added to $40 \mathrm{~mL}$ of $1.5 \% \mathrm{w} / \mathrm{v}$ PLA in dichloromethane and homogenized at $14,000 \mathrm{rpm}$ for 5-10 s. The primary emulsion was added to $250 \mathrm{~mL}$ of $1 \% \mathrm{w} / \mathrm{v}$ PVA solution and homogenized for 5-10 s and then stirred at $900 \mathrm{rpm}$ and washed in water. To make magnetic PLA, $1 / 4$ volume of $20 \% \mathrm{w} / \mathrm{v}$ suspension of magnetite was added to a $5 \% \mathrm{w} / \mathrm{v}$ solution of PLA in dichloromethane and homogenized. The PVA solution was added to 20 times the volume of primary emulsion. The PLA magnetite particles were cleaned by magnetic separation after 30 cycles. The magnetic crystals were $0.18 \pm 0.05 \mu \mathrm{m}$ with an $\mathrm{x}$-ray diffraction (XRD) grain size of $30 \mathrm{~nm}$. The magnetite-PLA particles were polydisperse $(1.7 \pm 0.5 \mu \mathrm{m})$ with surface area equal to $2 \mathrm{~m}^{2} / \mathrm{g}$. It appears that the PLA encapsulated magnetite aggregates rather 
than remaining as single magnetite particles. The magnetization was about half the pure magnetite phase (40 $\mathrm{Am}^{2} / \mathrm{kg}$ at saturation). The surface charge of magnetite was between 0 and $20 \mathrm{mV}$ at $\mathrm{pH} 7$ with a very strong $\mathrm{pH}$ dependence on charge. The strong $\mathrm{pH}$ dependence on charge suggests that the magnetite plays an important role in surface charge since the $\mathrm{pH}$ dependence on bare PLA microparticles is rather modest (Gomez-Lopera et al. 2001).

\section{PLA-PEG}

Li et al. (2000) used PEG6000 and PVA 130kDa, as well as PEG-PLA with 5-50\% PEG. They used emulsion evaporation of w/o/w to make microspheres to deliver the drug glucose oxidase. The glucose oxidase was dispersed in an initial water phase. The resulting mixture was emulsified in dichloromethane and ethyl acetate $(50 \mathrm{mg} / \mathrm{mL})$ at high speed for $60 \mathrm{~s}$, and then immediately homogenized in a PVA-water solution $(100 \mathrm{~mL})$. The organic solvent was extracted by adding $100 \mathrm{~mL}$ of $6 \%$ isopropanol and stirred for $3 \mathrm{~h}$, washed and lyophilized. Laser diffractometry revealed an average microsphere diameter of $1.92 \pm 0.21 \mu \mathrm{m}$, with more than $95 \%$ of the microspheres at $0.5-5 \mu \mathrm{m}$. Li et al. quantified the loss in glucose oxidase activity after each preparation step through lyophilization and found that $20-30 \%$ activity is lost during each emulsion step (two emulsion steps in the w/o/w method). Regardless of technique, a maximum of $0.96 \%$ protein ( $\mathrm{mg}$ protein $/ 100 \mathrm{mg}$ microparticles) was encapsulated, with the PEG-PLA working much better than PLA or PLGA alone. The protein retained a higher percentage of its activity when the PEG concentration was increased, showing $58 \%$ for PEG(5\%)-PLA and $90 \%$ for PEG-(30\%)-PLA. The efficiency of encapsulation (mg protein encapsulated per $100 \mathrm{mg}$ initial protein) also increased with the PEGylated copolymer over PLA or PLGA alone, where a maximum of $56 \%$ was encapsulated in PEG (10\%)-PLA. To determine the effect of organic solvent on denaturization, some dichloromethane was replaced with ethyl acetate. The results show that the activity of the protein was higher when ethyl acetate was used. When the EA:DCM volume was 0:5 the activity was $64.3 \%$ (activity of protein in U/mg per $\mathrm{U} / \mathrm{mg}$ in native solution), and when the EA/DCM volume was 3.5:1.5 the activity was $81 \%$. A ratio of 2.5:2.5 was best, though, for amount encapsulated and encapsulation efficiency, but this ratio also had very good specific activity (76\%). A gelatin stabilizer (not named) was used in the internal aqueous phase $(0-2 \% \mathrm{w} / \mathrm{w})$ but was not found to improve protein activity. The release of protein from PEG-PLA during in vitro testing showed a rapid release during the first day, then a long, slow release over a month. Polyacrylamide gel electrophoresis of the protein released from the particles showed no evidence of cleavage or aggregation (Li et al. 2000).

Liu and Deng (2002) did a parametric study of the effect of PLA-PEG nanoparticles on DNA loading using a water-in-oil-in-water $(\mathrm{w} / \mathrm{o} / \mathrm{w})$ method. They added hydrophilic polymer to the aqueous drug phase, added surfactant to the organic solution, varied polymer concentration in the organic phase, varied the second solvent (pentane, acetone, tetrahydrofuran, or ethyl acetate) 1:1 with the methylene chloride in the organic phase, varied the concentration of PEG, varied the PEG:PLA ratio, varied the external water phase volume and the hydrophilic PEG concentration added as stabilizer, and varied the DNA molecule. Aqueous DNA solution was dispersed into the organic solvent of the polymer and stirred for $2 \mathrm{~min}$ at room temperature. The resultant mixture was syringed into water and homogenized. This was added to $100 \mathrm{~mL}$ of $6 \%$ isopropanol to extract the solvent, then stirred for 5-6 h at room temperature. Then, it was centrifuged at 8,000-10,000 rpm for $10 \mathrm{~min}$, washed with deionized water, lyophilized, and stored at $-20^{\circ} \mathrm{C}$. PLA-PEG (PEG 6000MW) was synthesized by ring opening polymerization. 
The results were: (1) the loading efficiency peaked at 10\% PEG in PLA-PEG copolymer, (2) an increase in molecular weight of PEG saw a decrease in DNA-loaded sphere size but a higher loading efficiency, (3) ethyl acetate mixed 1:1 with methylene chloride as the organic phase produced the highest loading $(60 \%)$ and a smaller particle size $(1 \mu \mathrm{m})$, (4) increasing the concentration of polymer in the oil phase produced an increase in particle size and loading efficiency, (5) the increase in PEG in the external water phase decreased particle size from $4 \mu \mathrm{m}$ at $5 \%$ PEG to $1 \mu \mathrm{m}$ at $>25 \%$ and decreased loading efficiency, (6) the particle size peaked at $15 \%$ stabilizer in the external water phase, (7) the addition of sucrose $(0-10 \%)$ to the external water phase increased DNA encapsulation efficiency from 55 to 61\% (Liu and Deng 2002).

Quellec et al. (1998) used PEG-PLA or PLA-PEG-PLA, with the PEG (5 kDa)-PLA (45 kDa) prepared by ring-opening polymerization. First, $0.1 \mathrm{~mL}$ of human serum albumin (25$200 \mathrm{mg} / \mathrm{mL}$ ) was mixed with $2 \mathrm{~mL}$ of polymer solution in methylene chloride $(25-100 \mathrm{mg} / \mathrm{mL})$ in an ice bath under sonication $(15 \mathrm{~W}, 30 \mathrm{~s})$. Then, this was added to $4 \mathrm{~mL}$ of water containing surfactant $(0.6 \% \mathrm{w} / \mathrm{w})$ and pulse-sonicated $(40 \mathrm{~W}, 30 \mathrm{~s})$. This second emulsion was mixed with $100 \mathrm{~mL}$ of $0.1 \% \mathrm{w} / \mathrm{w}$ surfactant solution until the solvent was evaporated. They proved that no protein was found on the surface but rather was encapsulated within the polymer. After washing, PLA microspheres aggregated once the surfactant was removed but the PLA-PEG particles resuspended relatively well with $50-60 \%$ yield as opposed to $15-45 \%$ for PLA. Higher concentrations of polymer in the organic phase led to larger microparticles $(200 \mathrm{~nm}$ at $25 \mathrm{mg} / \mathrm{mL}$ and $450 \mathrm{~nm}$ at $100 \mathrm{mg} / \mathrm{mL}$ ) and increased protein loadings from 7 to $12 \%$. The point of zero charge was $-4 \mathrm{mV}$ but was more negative after encapsulation of the protein, which was interpreted as the reduction of PEG on the surface. The adsorption of water for these PLA-PEG microspheres reached saturation at $30 \mathrm{~min}$ at 28\% (Quellec et al. 1998).

The specific choice of a nanoparticle synthesis method depends strongly on the goal of the therapy. For therapies requiring the delivery of drugs, one must choose a method that allows a lipophilic or hydrophilic drug to be safely encapsulated within the microsphere. Also, size is important. Larger-sized particles have higher volume/mass ratios, naturally increasing the capacity for drug encapsulation. Smaller-sized particles have higher surface-area/mass ratios to increase surface receptors that might be needed to chemically differentiate abnormal tissue or cells. Smaller-sized particles, in general, have less magnetic component, making magnetic manipulation more difficult. For our current research goals, we require particles $<5 \mu \mathrm{m}$ to avoid arterial occlusion, and a surface properly configured to prolong vascular survival (high PEG surface density). However, the particles must be large enough to enable magnetic manipulation ( $>3 \mathrm{emu} / \mathrm{g}$ ), and the polymer must be compatible with high drug-encapsulation efficiencies and release of active drug. These requirements favor several of the outlined methods, but the best ones can be determined only when one applies a specific medical goal. Recently, we submitted a National Institutes of Health (NIH) R21 application describing the preparation of PLA or PLGA magnetic microspheres to encapsulate recombinant tissue plasminogen activator (rtPA) for clot lysis. This application outline is provided in Appendix B. The methods of Morita et al. (2000b) and Gref et al. (2001) were chosen to test the proposal hypothesis. However, the method of Liu and Deng (2002) may also be appropriate in that protein denaturization can be reduced with the $\mathrm{w} / \mathrm{o} / \mathrm{w}$ technique and the resulting microparticle size is appropriate. In an NIH R01 application submitted in 2002, we describe the method of Hafeli et al. (2001) for the conjugation of rhenium- 
188 to bare PLA microspheres for head and neck solid tumor irradiation. This application outline is attached as Appendix C. 
Table 10. Synthesis parameters for biodegradable particles.

\begin{tabular}{|c|c|c|c|c|c|c|c|c|c|c|c|}
\hline & MW PLGA, & MW PLA, & 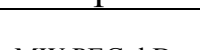 & Ratio & & & & & & & \\
\hline $\begin{array}{l}\text { Author } \\
\text { Gref et al. } 1994\end{array}$ & $\begin{array}{c}\mathrm{kDa} \\
45(3: 1)\end{array}$ & & $\frac{\text { MW PEG, kDa }}{5-20}$ & $\begin{array}{l}\text { PLA:PLGA:PEG } \\
\text { 10\% PEG (36::5) }\end{array}$ & $\begin{array}{l}\text { Solvent } \\
\text { Ethyl acetate or methylene chloride, } \\
25 \mathrm{mg} \text { polymer/2 } \mathrm{mL}\end{array}$ & 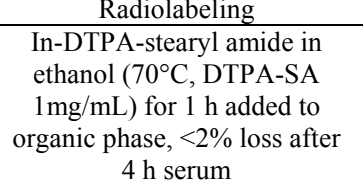 & $\begin{array}{l}\text { Mixing } \\
\text { Vortex, sonicate }\end{array}$ & $\begin{array}{l}\text { Distribution } \\
\text { Monodisperse }\end{array}$ & $\begin{array}{c}\text { Size } \\
140 \mathrm{~nm}\end{array}$ & $\begin{array}{l}\text { Animal rests } \\
\text { MEe, higher MW } \\
\text { circ the longer } \\
\text { cirulation, } 20 \% \text { left at } \\
1.5 \mathrm{~h}\end{array}$ & Comments \\
\hline Stolnik et al. 2001 & & $2-25$ & 2 or 5 & 2:0:5-25:0:5 & $\begin{array}{l}\text { Acetone (+copolymer) in water, } \\
\text { water in accotone }(+ \text { copolymer), } \\
4 \% \mathrm{wW} / \mathrm{v} \text { copolymer in organic phase, } \\
10 \mathrm{mg} \text { copolymer } / \mathrm{mL} \text { acetone, } 5 \\
\mathrm{~mL}\end{array}$ & $\begin{array}{l}\text { In-oxime complex }(50 \mu \mathrm{\mu L}, 37 \\
\mathrm{MB} / \mathrm{mL} \text { ) added to actone } \\
\text { phase }\end{array}$ & & $\begin{array}{c}\text { Polydisperse, } \\
\text { PI=0. 19-0.39, 25-76, } \\
\text { nmby oilin-water, } \\
32-390 \text { by water-in- } \\
\text { oil depending on } \\
\text { PLA:PEG ratio }\end{array}$ & & $\begin{array}{l}\text { Rat (o/w particles } \\
\text { only), above } 50-70 \mathrm{~nm} \\
25 \% \text { particles still in } \\
\text { blood after } 3 \mathrm{~h}\end{array}$ & $\begin{array}{l}\text { Filtered particles through } 200 \mathrm{~nm} \text { filter, } \\
\text { smaller PPA:PEG smaller particles, small } \\
\text { dependence on copolymer in acetone conc. }\end{array}$ \\
\hline $\begin{array}{l}\text { deJaeghere, from Stolnik } \\
\text { et al. 2001 }\end{array}$ & & 25 & $2-10$ & PLA:PEO:PLA & (a) & & & & $300 \mathrm{~nm}$ & & \\
\hline $\begin{array}{l}\text { Riley-1999 from Stolnik } \\
\text { et al. } 2001\end{array}$ & - & $3-110$ & 5 & & & & & & & & $\begin{array}{l}\text { Large MW PLA made physically unstable } \\
\text { particles. }\end{array}$ \\
\hline $\begin{array}{l}\text { Stolnik-1994 from } \\
\text { Stolnik et al. } 2001\end{array}$ & & & Poloxamer 908 & & & & & & & $\begin{array}{l}\text { Better than Stolnik et } \\
\text { al } 201 \text { resuls }\end{array}$ & \\
\hline Gref et al. 1995 & & 45 & 5 & & $\begin{array}{l}0.6 \mathrm{w} \% \text { surfactant (like PVA or } \\
\text { cholic acid), surfactant varied }\end{array}$ & As in Gref et al. 1994 & & $\begin{array}{l}\text { Monodisperse, } \\
\mathrm{PI}=0.05-0.1 \\
\end{array}$ & & 2-D PAGE, mice & $\begin{array}{l}\text { Zeta potential (-1)-1, PLA at }-4-12 \text {, cholic } \\
\text { acid good, more surfactant smaller size. }\end{array}$ \\
\hline Fu et al. 1999 & 25 & & - & - & Methylene chloride & & Sonicate/homogenize & & - & & $\begin{array}{l}\text { Study found that } 1 / 3 \text { protoin denatured upon } \\
\text { encapsulation, stabilizer helped. }\end{array}$ \\
\hline $\begin{array}{l}\text { Liu and Deng } 2002 \\
\end{array}$ & - & $5.3-102.2$ (a) & $6-62.4$ & & $\begin{array}{l}\mathrm{W} / \mathrm{O} / \mathrm{W} \text {, methylene chloride plus } \\
\text { second solvent }\end{array}$ & No & $\begin{array}{l}\text { Stirred, homogenized, } \\
\text { stirred }\end{array}$ & Unknown & $0.5-4.3 \mu \mathrm{m}$ & No & 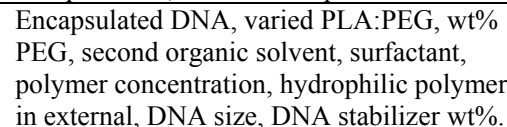 \\
\hline Donini et al. 2002 & & $\begin{array}{c}\text { Methacrylic } \\
\text { acid }\end{array}$ & $0.2-1$ & 1:01 & $\begin{array}{l}\text { Water with cross-linker, } \\
\text { photoinitiator, and UV }\end{array}$ & No & Not stated & & $<50-300 \mathrm{~nm}$ & No & $\begin{array}{l}\text { Larger PEG length made smaller more } \\
\text { uniform particles. }\end{array}$ \\
\hline Mosquiera et al. 2001 & & $\begin{array}{l}45 \text { (poly(rac- } \\
\text { lactic acid)) }\end{array}$ & 5 or 20 & $\begin{array}{l}\text { Synthesized } \\
\text { according to ref } 18, \\
10-30 \% \text { PEG w/w } \\
\text { total polymer in } \\
\text { acetone }\end{array}$ & $\begin{array}{l}\text { Acetone with lecithin and Miglyol } \\
810 \mathrm{~N}\end{array}$ & H-3 labeled PLA & Not stated & Broad & $170-200 \mathrm{~nm}$ & $\begin{array}{l}\text { 30\% in blood after } 1 \mathrm{~h} \\
\text { with 20kDa PEG }\end{array}$ & $\begin{array}{l}\text { rac-lactide is amorophous compared to } \\
\text { crystalline D- or L-lactide and thus degrades } \\
\text { faster. }\end{array}$ \\
\hline $\begin{array}{l}\text { Cui and Schwendeman } \\
2001\end{array}$ & $\begin{array}{l}9.44 \text { avg PLGA, } \\
\text { end capped } \\
\text { (50:50) }\end{array}$ & - & - & 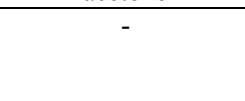 & $\begin{array}{l}\mathrm{O} / \mathrm{W} \text { solvent evap., methylene } \\
\text { chloride }\end{array}$ & No & $\begin{array}{c}\text { Vortex/ } \\
\text { stir }\end{array}$ & Broad & $>1000 \mathrm{~nm}$ & No & Large microspheres. \\
\hline Mosqeuira et al. 2000 & & $9-251$ & & & $\begin{array}{c}\mathrm{O} / \mathrm{W} \text { solvent evaporation, } \\
\text { methylene chloride }\end{array}$ & No & & Broad & $180-700 \mathrm{~nm}$ & No & Varied surfactant, PLA MW. \\
\hline Morita et al. 2000 & $\begin{array}{c}10 \text { and } 20 \\
(50: 50)\end{array}$ & 20 & 6 and 70 & & $\begin{array}{l}\mathrm{S} / \mathrm{O} / \mathrm{W}, \text { PEG-protein lyophilized } \\
\text { and dispersed in PLA }\end{array}$ & No & Homogenized, stirred & & $>30 \mu \mathrm{m}$ & & $\begin{array}{l}\text { Encapsulated HRP and bovine superozide } \\
\text { dismutase. }\end{array}$ \\
\hline Li et al. 2000 & & (a) & $\begin{array}{l}6 \text { or PEG-PLA } \\
\text { of } 5-50 \% \text { PEG }\end{array}$ & & $130 \mathrm{kDa}$ PVA & No & Homogenized & Broad & $1.9 \pm 0.2 \mu \mathrm{m}$ & No & $\begin{array}{l}\text { Glucoso oxidase encapsulation and release. } \\
50 \% \text { protein encapsulated } 40-50 \% \text { activity } \\
\text { lost during encapsulation. }\end{array}$ \\
\hline McGee et al. 1995 & 9 or $90(50: 50)$ & or 300 PLA(a) & None & & Silicone with surfactants & No & Homogenized & Broad & & No & $\begin{array}{l}\text { Encapsulated ovalalbumin, varied volume } \\
\text { of dichloromethane, surfactants, } \\
\text { temperature of emulsion solution. }\end{array}$ \\
\hline $\begin{array}{l}\text { Gomez-Lopera et al. } \\
2001\end{array}$ & & 106 & - & & Dichloromethane & No & Homogenized & Broad & $1.7 \pm 0.5 \mu \mathrm{m}$ & No & Studied properties of magnetite-PLA. \\
\hline Quelllic et al. 1998 & & 45 & 5 & & $\mathrm{~W} / \mathrm{O} / \mathrm{W}$, methylene chloride, water & & Sonicate & Monomodal & $\begin{array}{l}200-450 \mathrm{~nm}, \\
\text { parameter-dependent }\end{array}$ & No & Focused on PLA-PEG/PLA comparison. \\
\hline Dunn et al. 1997 & $63(75: 25)$ & - & $\begin{array}{c}6.7-25 \\
\text { (poloxamer) }\end{array}$ & - & Acetone & In-111 and I-125 & Mixing & Narrow & $99-122 \mathrm{~nm}$ & $\begin{array}{l}\text { Yes (rat, rabbit), } \\
\mathrm{T} / 2=2.5 \mathrm{~h}\end{array}$ & $\begin{array}{l}\text { Compared results to PS, found little } \\
\text { didfference, copolymerized particles } \\
\text { performed equally to preformed particles } \\
\text { incubated in poloxamer. }\end{array}$ \\
\hline Arias et al. 2001 & PECA & & & & Water & & Mixed & Narrow & $144 \pm 15 \mathrm{~nm}$ & No & $\begin{array}{l}\text { Used poly(ethylene-2-cyanoacrylate) as } \\
\text { biodegradable polymer. Surface charge was } \\
-40 \mathrm{mV} \text {. }\end{array}$ \\
\hline O'Hagan et al. 1991 & & $9(50: 50)$ & & & Dichloromethane & & Homogenized & Broad & $5.34 \mu \mathrm{m}$ avg & In-111 & $\begin{array}{l}\text { Phagocytosed particles can deliver } \\
\text { controlled release of antigen. }\end{array}$ \\
\hline
\end{tabular}

(a) Data in source cited are unclear. 



\section{PREVIOUS CLINICAL TRIALS}

\section{a. Therapeutic Drugs}

During the last 25 years, a small number of groups have tried to use magnetic particles for active drug targeting (Widder et al.1978, Gupta and Hung 1990, Gupta and Hung 1993, Häfeli et al. 1994, Lübbe et al. 1996a, 1996b). In one such experiment, squamous cell carcinoma in New Zealand white rabbits were treated using 100-nm magnetic particles coated with starch polymer and bound to mitoxantrone ( $20 \%$ and $50 \%$ of the systemic dose). The particle drug complex was administrated by injecting it intra-arterially (femoral artery) or intravenously (ear vein), while the external magnetic field was focused on the tumor. The intra-arterial delivery of particles resulted in complete and permanent remission of the squamous cell carcinoma in contrast to the control group (no treatment) and the intravenously delivered particles, with no signs of toxicity. The intratumoral accumulation of magnetic particles was visualized both histologically and by MRI (Alexious et al. 2000).

Kuznetsov et al. (2001) injected 10-nm magnetite particles loaded with photosense or teraphthal encapsulated in liposomes into the tail veins of laboratory mice. $\mathrm{A} \mathrm{SmCo}_{5}$ permanent magnet with $0.24 \mathrm{~T}$ field on the surface was positioned next to a carcinoma tumor on a rear extremity. Controlled animals were injected with photosense containing non-magnetic liposomes (without ferromagnetic particles), then the tumor zone was irradiated. The tumor was destroyed only by the magnetized photosense. Kuznetsov et al. have also used sub-micrometer ferromagnetic particles containing muscle relaxant drugs encapsulated into liposomes for selective relaxation of n-choline receptors of skeletal muscles. This application has the potential to lead to local muscle relaxation without the need for artificial lung ventilation, and avoid heart function disorder. The kinetics of the drug release from liposomes in blood and blood serum was studied using liposomes loaded with bromophenol blue. When intravenously injected, a dose of $115 \mu \mathrm{g} / \mathrm{kg}$ of diadony encapsulated into magnetic liposomes reduced the amplitudes of nerve muscle by $70 \%$ in the presence of the magnetic field and 15\% without a magnet. Similarly, a dose of $20 \mu \mathrm{g} / \mathrm{kg}$ Diperony-loaded magnetic liposomes reduced the amplitude by $45 \%$ with a magnet and $5 \%$ without.

Mikhaylyk et al. (2001) conducted similar research with nanomaterials based on metallic iron and magnetite. Different surface properties were used as potential targeting in adult male Wistar rats' (weighing $\sim 150 \mathrm{~g}$ ) glial brain for tumor diagnosis and treatment. The iron preparations were injected at a dose of $12 \mathrm{mg} \mathrm{Fe} / \mathrm{kg}$ into the tail vein of the rats with and without a magnetic field $(0.075 \mathrm{~T})$ imposed on the brain region. Injection of magnetite-dextran nanoparticles into the carotid artery resulted in penetration of particles into the brain tumor and peritumoral tissue of the rats bearing glial brain tumor. MRI images showed pronounced signal loss in the tumor region.

Other work by Alexiou et al. (2001) reported the use of magnetic particles coated with starch polymers as biocompatible medical drug carriers. The starch coating of the particles allowed binding to the anticancer agent mitoxantrane. Rabbits with tumors in the medial portion of the hind limb were injected intra-arterially ( $50 \%$ of systemic dose) with the mitoxantrone bound to magnetic particles and targeted by an external magnetic field. The tumor size decreased and 
showed complete remission within 15 days. The control group, which was not treated, showed growth of the tumor.

\section{b. Therapeutic Radionuclides}

In radiation therapy, the main objective of targeted drug delivery is to reduce tumor host imbalance by altering the distribution or the effects of drugs such that tumor cells are damaged substantially more than normal cells. There are two general conditions that apply to most tumorbearing patients. (1) Biological activities and surface properties of the malignant cells cause imbalance in tumors and host recognition and elimination, and (2) biochemical differences between tumor and host cells are always minimal and frequently quantitative rather than qualitative (Hurwitz et al. 1975). These conditions give rise to difficulties in overcoming tumorhost imbalance using a non-targeting agent. It is of greater importance to be able to selectively target the tumor in order to reduce the resulting toxic side effects from generalized distribution and to be able to use a much smaller dose, which would further lead to reduction of toxicity. For these reasons, targeted radiation delivery using magnetic particles is limited to solid tumors. However, if used in conjunction with chemical or biological targeting agents (e.g., EGFR, MAbs), magnetic particles tagged with therapeutic radionuclides can offer distinct advantages in treating metastasized or dispersed tumor cells.

The important physical variables to consider in radiation therapy include the radionuclide halflife, the type, energy, and branching ratio of particulate radiation and the gamma-ray energies and abundances (Mausner and Srivastava 1993). A large number of radionuclides exist (Table 11), emitting nuclear particles with a range of path lengths from nanometers ( $\alpha$-particles) to millimeters ( $\beta$ and $\gamma$ rays) (Wheldon and O'Donoghue 1990). While some radiation therapy approaches utilize MAbs as carriers of the radionuclides (Larson 1991, Goldenberg and Griffiths 1992), others use magnetic particles labeled with radionuclides and guided by an external magnetic field into close proximity to the tumor (Ehrhardt and Day 1987, Häfeli et al. 1994, Gupta and Hung 1993, Lübbe et al. 1996b).

Table 11. Physical characteristics of radionuclides of interest for targeted radiotherapy.

\begin{tabular}{|c|c|c|c|}
\hline Radionuclide & Half-life & Particles emitted & $\begin{array}{c}\text { Mean tissue } \\
\text { range }\end{array}$ \\
\hline${ }^{131} \mathrm{I}$ & 8 days & $\beta$ & $0.8 \mathrm{~mm}$ \\
\hline${ }^{90} \mathrm{Y}$ & 2.7 days & $\beta$ & $5 \mathrm{~mm}$ \\
\hline${ }^{67} \mathrm{Cu}$ & 2.6 days & $\beta$ & $0.6 \mathrm{~mm}$ \\
\hline${ }^{212} \mathrm{Bi}$ & 1 hour & $\beta, \alpha$ & $0.05-5 \mathrm{~mm}$ \\
\hline${ }^{125} \mathrm{I}$ & 60 days & Auger electrons & $\sim 1 \mathrm{~mm}$ \\
\hline${ }^{77} \mathrm{Br}$ & 2.4 days & Auger electrons & $\sim 1 \mathrm{~mm}$ \\
\hline${ }^{188} \mathrm{Re}$ & 17 hours & $\beta$ & $3-4 \mathrm{~mm}$ \\
\hline${ }^{32} \mathrm{P}$ & 14 days & $\beta$ & $3-4 \mathrm{~mm}$ \\
\hline
\end{tabular}

Source: Modified from Wheldon and O’Donoghue (1990). 
To target drug delivery to a specific site, Goodwin et al. (1999) used magnetic particles on a swine. The magnetic particles were formed using a high-energy milling process incorporating activated carbon into metallic iron powder to produce a microparticle composite with a 75:25, $\mathrm{Fe}: \mathrm{C}$ ratio (particle size range from 0.5 to $5 \mu \mathrm{m}$ ). For monitoring short-term retention, the magnetic particles were labeled with technecium-99M $\left({ }^{99 \mathrm{~m}} \mathrm{Tc}, 8.5 \mathrm{mCi}\right)$. A neodymium permanent magnet was placed outside the body positioned at a pre-selected site in the liver. The magnetic field strength at the site ranged from 0.025 to 0.10 Tesla. Then, $200 \mathrm{mg}$ of the magnetic particles suspended in $200 \mathrm{~mL}$ of sterile saline were administrated at $2 \mathrm{~mL} / \mathrm{min}$ intraarterially. Whole-body gamma camera images were generated; selected results are shown in Table 12. Approximately $42 \%$ of the total activity was found in a very localized region of the liver, and almost $67 \%$ of the total activity was found in the targeted lobe of the liver.

Table 12. Analysis of radiographic images of entire swine following ${ }^{99 \mathrm{~m}}$ Tc-MTC administration at $\mathrm{t}=60$ and $120 \mathrm{~min}$ from start of infusion. ${ }^{99 \mathrm{~m}} \mathrm{Tc}$-magnetic particles administrated intra-arterially through a segmental branch of the hepatic artery in the presence of a magnetic field.

\begin{tabular}{|l|c|c|}
\hline \multicolumn{1}{|c|}{ Organ } & $\begin{array}{c}\text { Percent of Total Activity } \\
\mathrm{t}=60 \mathrm{~min}\end{array}$ & $\begin{array}{c}\text { Percent of Total Activity } \\
\mathrm{t}=120 \mathrm{~min}\end{array}$ \\
\hline Bladder & 4.0 & 4.2 \\
\hline Salivary 1 and 2 & 21.2 & 22.1 \\
\hline Cardiac Pool & 8.2 & 7.1 \\
\hline Whole Liver & 66.6 & 63.1 \\
\hline Targeted Liver & 41.7 & 36.9 \\
\hline
\end{tabular}

Source: Modified from Goodwin et al. (1999).

Recently, Häfeli et al. (2001) developed a radiotherapeutic drug delivery system based on magnetic microparticles $(0.5$ to $5 \mu \mathrm{m})$ composed of up to $80 \%$ metallic iron. The remaining weight consists of activated carbon labeled with rhenium-188 $\left({ }^{188} \mathrm{Re}\right)$. The simple one-step radiolabeling of magnetic microparticles with ${ }^{188} \mathrm{Re}$ at $99^{\circ} \mathrm{C}$ in the presence of tin chloride resulted in $>95 \%$ labeling efficiency. The $\beta$-emitting radionuclide ${ }^{188} \mathrm{Re}$ was chosen because the $\beta^{-}$radiation (maximum energy $2.12 \mathrm{MeV}$ ) is effective in penetrating to a range of $11 \mathrm{~mm}$ and can destroy tumor cells up to a maximum range of $2.6 \mathrm{~mm}$ in tissue. In addition ${ }^{188} \mathrm{Re}$ has a $\gamma$-line at $155 \mathrm{keV}$. This energy is close to that of ${ }^{99 \mathrm{~m}} \mathrm{Tc}$ and can be imaged easily with a $\gamma$-camera.

Similarly, Ehrhardt and Day (1987) used glass microspheres containing ${ }^{31} \mathrm{P}$ or ${ }^{89} \mathrm{Y}$ that can be activated by neutron bombardment to ${ }^{32} \mathrm{P}$ or ${ }^{90} \mathrm{Y}$ for radiotherapy of liver tumor. When injected through a catheter into the hepatic artery, the 15- to 30- $\mu$ m-diameter microspheres became trapped in the tumor capillary bed. The short-range highly ionizing beta emissions delivered large local radiation doses with little irradiation to neighboring organs. Trials in rabbits and dogs have proven higher radiation dose to tumor can be achieved than with external beam therapy.

\section{c. Magnetic Resonance Imaging (MRI)}

Early work in magnetic resonance (MR) contrast agents consisted of large non-biodegradable superparamagnetic iron oxide, but this was later replaced by small biodegradable magnetite particles (3-12 nm) encapsulated in dextran. According to Pouliquen (2001), these particles are coated with or encapsulated in suitable biocompatible polymers such as dextran for three main 
reasons: (1) to overcome the natural tendency of magnetic nanocrystals to aggregate and settle due to van der Waals interaction, (2) to modify the high zeta potential of nanocrystals, which leads to their opsonization, and (3) to control relaxitivities (relaxation times) of the particles. In addition to this, surface properties of magnetic nanocapsules also strongly affect their uptake by the RES.

Traditionally, MR contrast agents are based on paramagnetic metal chelates (mostly Gd chelates) (Weinmann et al. 1984). However, paramagnetic metal chelates usually have short blood residence and are generally non-specific. On the other hand, micro- and nanoparticulate materials offer some tissue specificity and the possibility of targeting, and hence much attention is focused on nanoparticulate magnetic contrast agents. Most notably, dextran-coated nanometer-size superparamagnetic iron oxide particles are phagocytosed by the RES, and have proved very useful for detection and characterization of hepatic neoplasms. Magnetic nanoparticles used or investigated as MR contrast agents are composed of magnetite $\left(\mathrm{Fe}_{3} \mathrm{O}_{4}\right)$ or maghemite $\left(\gamma-\mathrm{Fe}_{2} \mathrm{O}_{3}\right)$ nanocrystals encapsulated in a dextran matrix (Tanimoto 2001).

Päuser et al. (1997) also intensively investigated the application of superparamagnetic iron oxide particles encapsulated with oleic acid and incorporated within the lipid phase of different types of liposomes as markers in non-invasive magnetic resonance imaging. In contrast to conventional pharmacokinetic procedures, non-invasive imaging techniques allow the investigation of the carrier kinetics in one animal at various times and thus help to reduce, drastically, the number of animals needed; previous imaging approaches using positron emission tomography and fluorescence were expensive and elaborate (van-Leengoed et al. 1994, Oku et al. 1995).

\section{B. PROGRAMATIC PROGRESS}

Groups of scientists and engineers in five separate Argonne Divisions: Chemical Engineering, Energy Technology, Material Science, Biosciences, and Mathematics and Computer Sciences, have been working together with the Cleveland Clinic Foundation and The University of Chicago Department of Neurology and Surgery (Neurosurgery) to develop novel and potentially versatile drug delivery system. The team consists of experts in nanoparticle synthesis and characterization, magnetic field design, measurement of the magnetic properties of nanoparticles, computational modeling of flow hydrodynamics, nanoparticle flow simulation/testing and suspension modeling, molecular biology, neurology, pathology, and surgery. The following summary describes work performed over the past two years.

\section{BIODEGRADABLE MICROPARTICLE SYNTHESIS}

We have conducted a series of experiments to produce magnetic microspheres with a uniform size distribution $<5 \mu \mathrm{m}$ by following the solvent evaporation method. In this method, a solution of PLA or PLGA and chloroform (or other volatile solvent with comparable polarity to chloroform) is added to a homogenized solution of $1 \%$ of polyvinyl alcohol (PVA) in water, followed by continued mixing, centrifugation, and washing of the particles. We investigated seven experimental variables: PLA-chloroform ratio, mixing speed, injection method, PVA molecular weight, effect of PEG, effect of surfactants, and effect of ultrasonic agitation. As 
shown in Figure 8, the results are highly encouraging. Most of the particles were about $1.0 \mu \mathrm{m}$ in diameter, and there were also some in the range of 3-5 $\mu \mathrm{m}$ that can be filtered. The full data is being prepared as a separate Argonne National Laboratory report (Kaminski 2003).

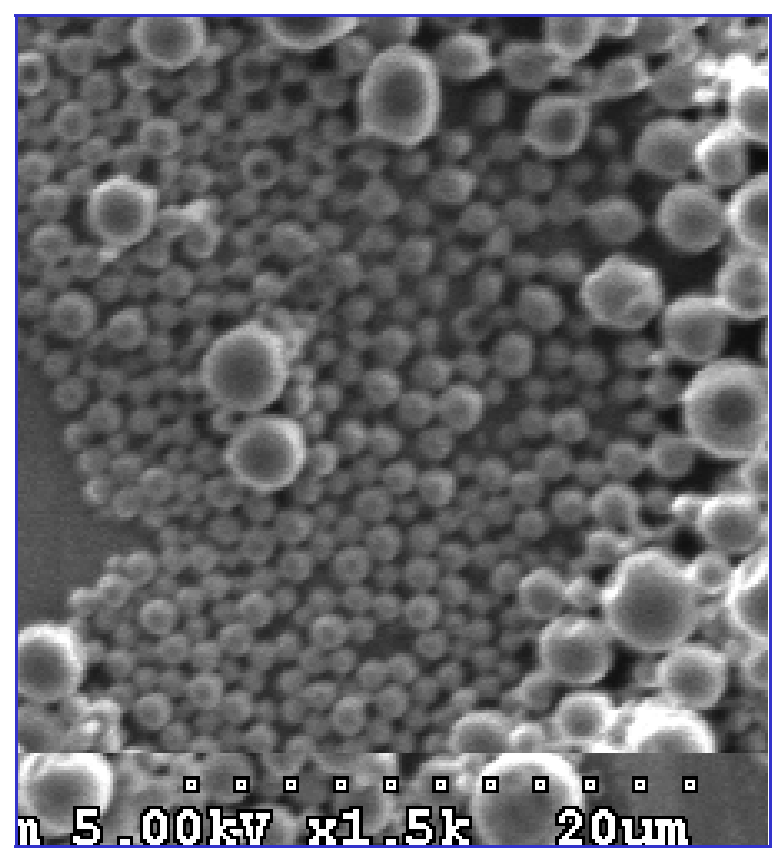

Figure 8. SEM image of PLA-based microspheres. Image has been cropped to show scale bar clearly. Scale bar is shown as the line of white squares at the bottom of the image spanning $20 \mu \mathrm{m}$.

\section{MAGNET CONFIGURATIONS}

The literature describes some magnetic separators for cell counting based on quadrupole magnets and we discussed briefly the use of permanent magnets for preclinical and clinical investigations. For our purpose we have briefly studied the applicability of simple designs using permanent flatplate, bar and donut-shaped $\mathrm{NdFeB}$ magnets. The magnetic field profiles from these geometries are shown in Figure 9. The profiles shown in these figures were determined with a Hall probe but will generally be determined by the computational magnetic field model described in the next section. In addition, Dexter Magnetic Technologies, Inc., provided several magnet conceptual plots for our evaluation and these have been included here. 


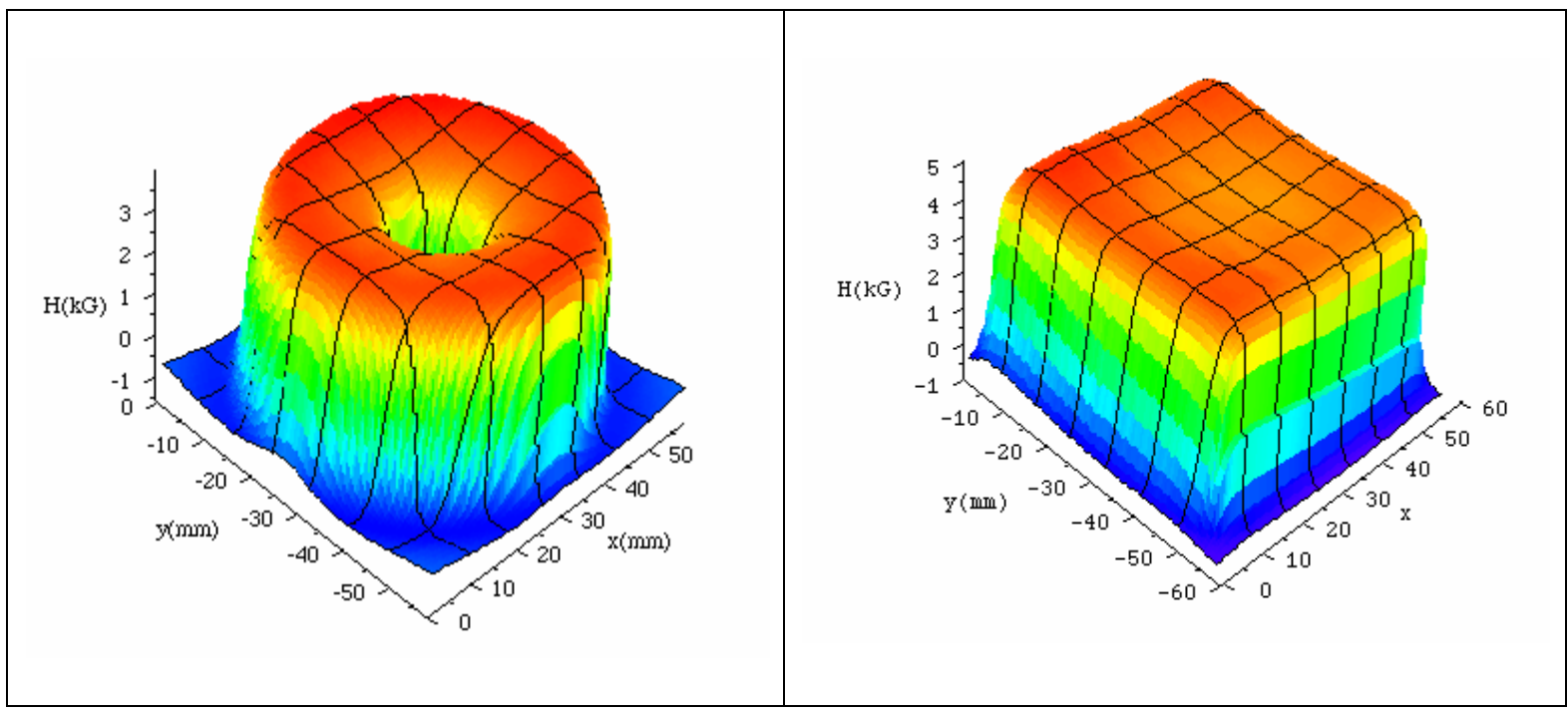

Figure 9. Two-dimensional magnetic field strength $(10 \mathrm{kG}=1 \mathrm{~T})$ profile for NdFeB magnets.

The type of field profile shown on the contour plot in Figure 10 was provided by Dexter Magnetic Technologies. The geometry (concept 1) could be scaled and optimized to provide the highest field gradient at 1.5-2 inches. This model utilizes two permanent magnets in order to minimize weight. Currently, the unit weight should be less than 30 pounds. The deep blue in the contour plot represents the starting point for collection. At this point, where the flux density is 0 Tesla, the gradient is on the order of $7 \mathrm{Tesla} /$ meter. Thus, the line segment originates from this point, and the same line represents the direction of travel for a particle affected by the magnetic field. The average gradient as the particle traverses the line is on the order of $20 \mathrm{Tesla} / \mathrm{meter}$. Also, the "funneling" characteristic of the magnetic profile shows that a magnetic particle will always travel in the direction of the highest gradient, all particles should collect at the point between the two magnets.

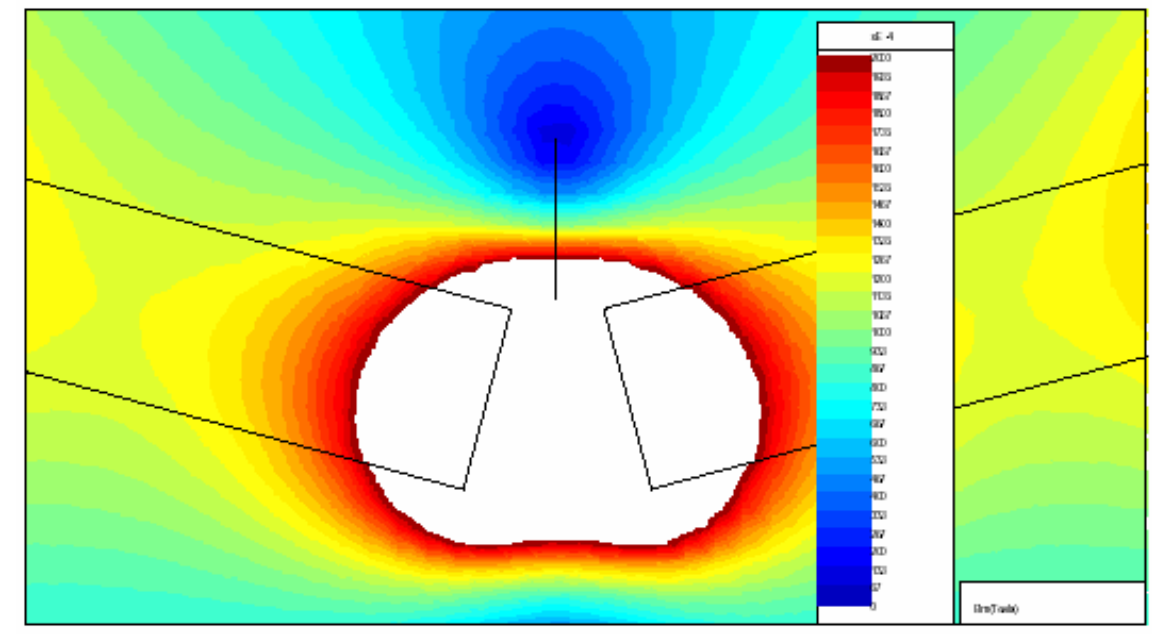

Figure 10. Flux density contours for concept 1 . The graduated legend is in units of Tesla and spans from 0 to 0.2 . 
Figure 11 shows the actual and magnified flux line for a second concept and Figure 12a the flux density about the line of interest; its gradient is shown in Figure 12b. The centerline of interest is $1.5 \mathrm{in}$. long in this design. The ordinate for Figure 12a is $\mathrm{B}_{\mathrm{m}}$ in Tesla while the abscissa is distance in meters. The ordinate and abscissa for Figure $12 \mathrm{~b}$ is $\mathrm{dB}_{\mathrm{m}} / \mathrm{dD}$ in $\mathrm{T} / \mathrm{m}$ and distance in meters, respectively. Figures 14, 16, and 18 follow similarly.

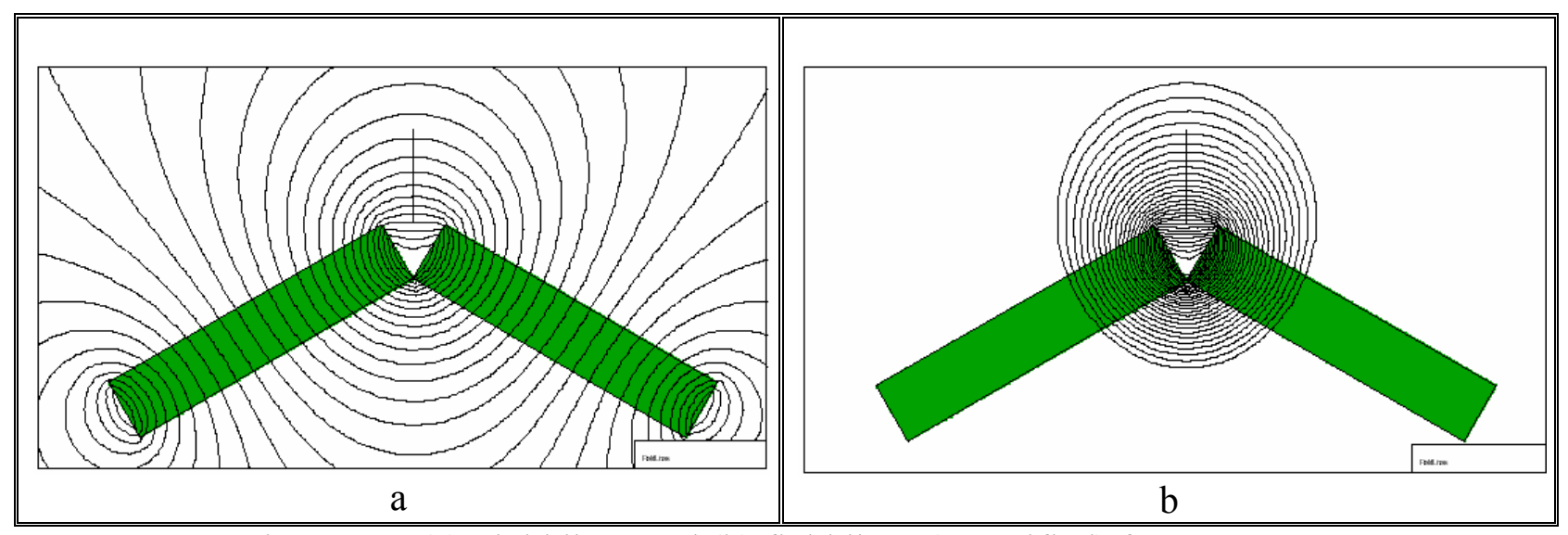

Figure 11. (a) Field lines and (b) field lines (magnified) for concept 2.
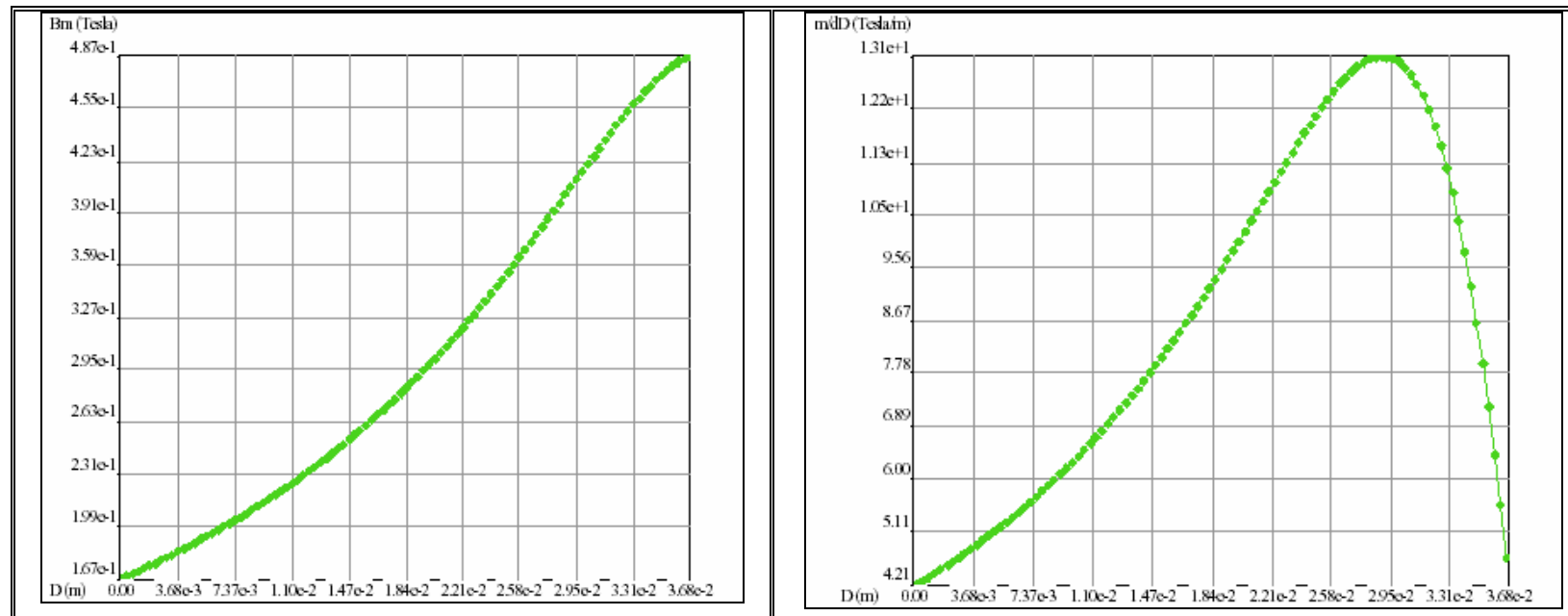

Figure 12. (a) Flux density along centerline in Figure 11 and (b) flux density along centerline (differentiated) for concept 2 .

A third concept shows the flux lines in Figures 13a and 13b, the flux lines magnified to view lines about the active area. The flux density and gradient about the line of interest are shown in Figure 14. This is a very simple, proven, configuration that is utilized in magnetic separators. This configuration will provide excellent retention once the particle reaches its static location. The line of interest is again $1.5 \mathrm{in.}$ long in this design. 


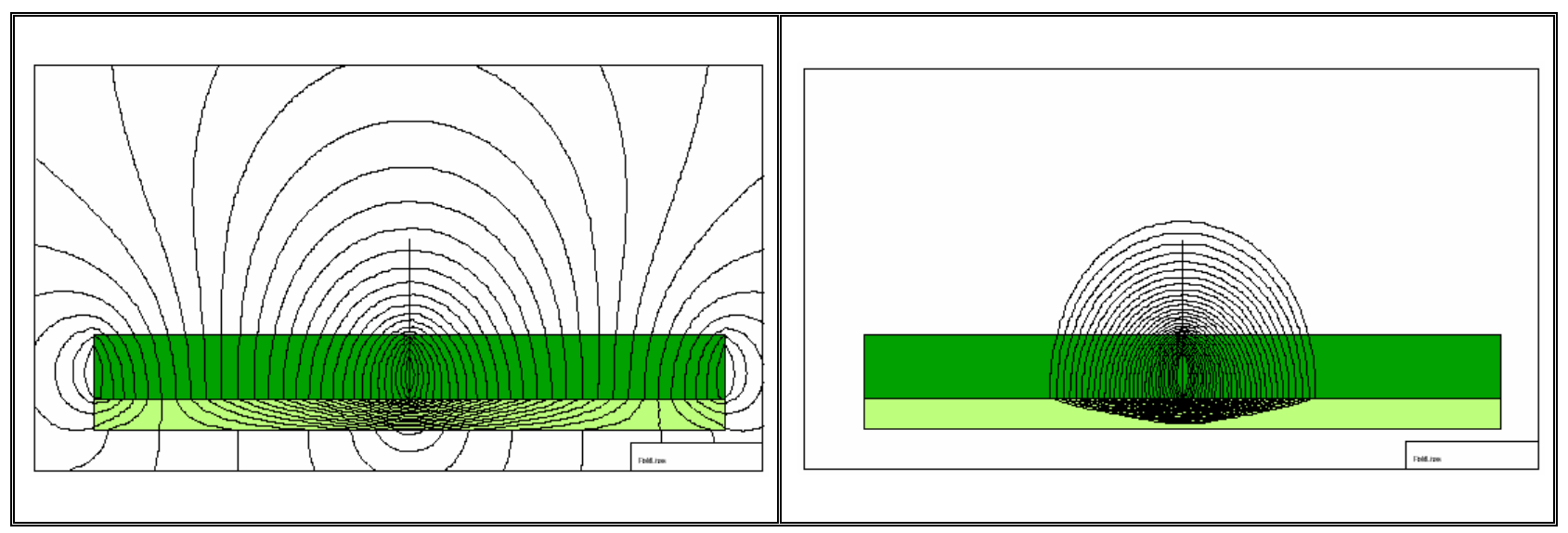

Figure 13. (a) Field lines and (b) field lines (magnified) for concept 3.
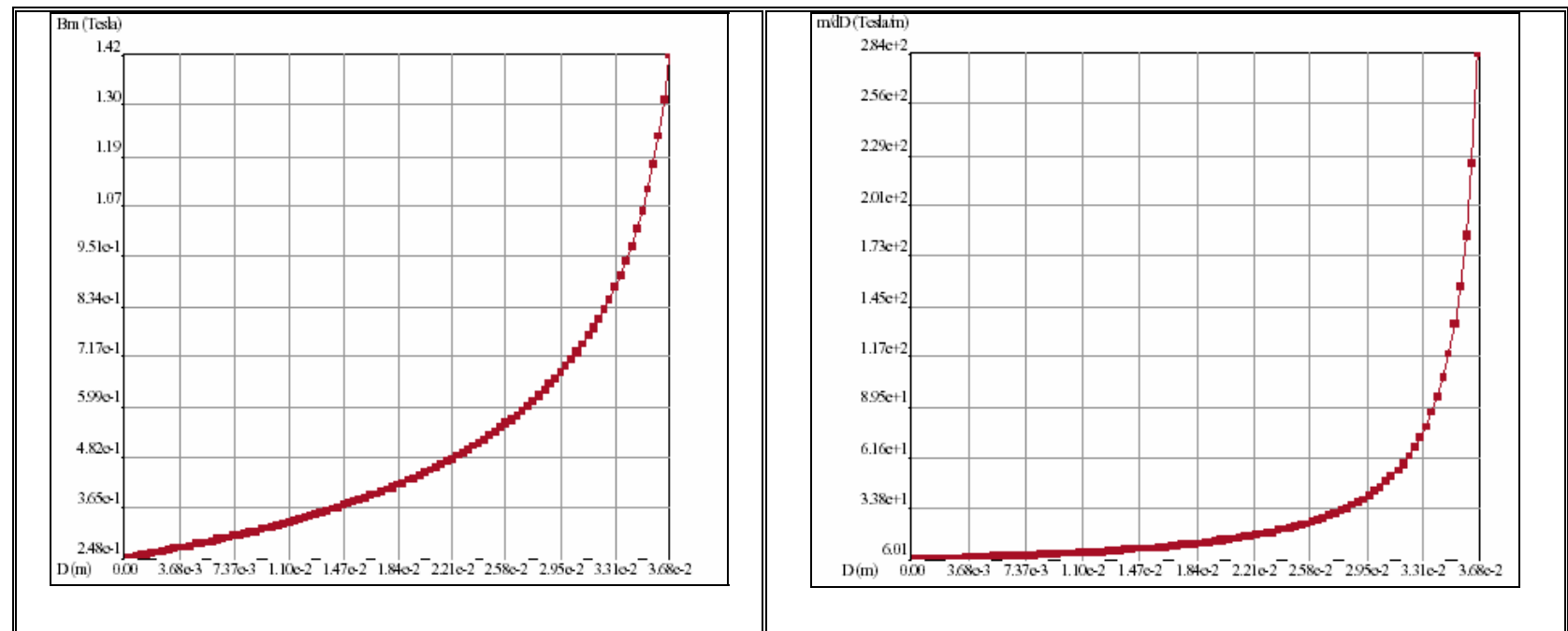

Figure 14. (a) Flux density along centerline and (b) flux density on line (differentiated) for concept 3.

The actual and magnified flux lines for the fourth concept are shown in Figure 15. The flux density about the line of interest and its gradient are shown in Figure 16. This concept is essentially the same as configuration 3 , but slightly modified to produce a larger gradient. The line of interest is again $1.5 \mathrm{in}$. long in this design. 


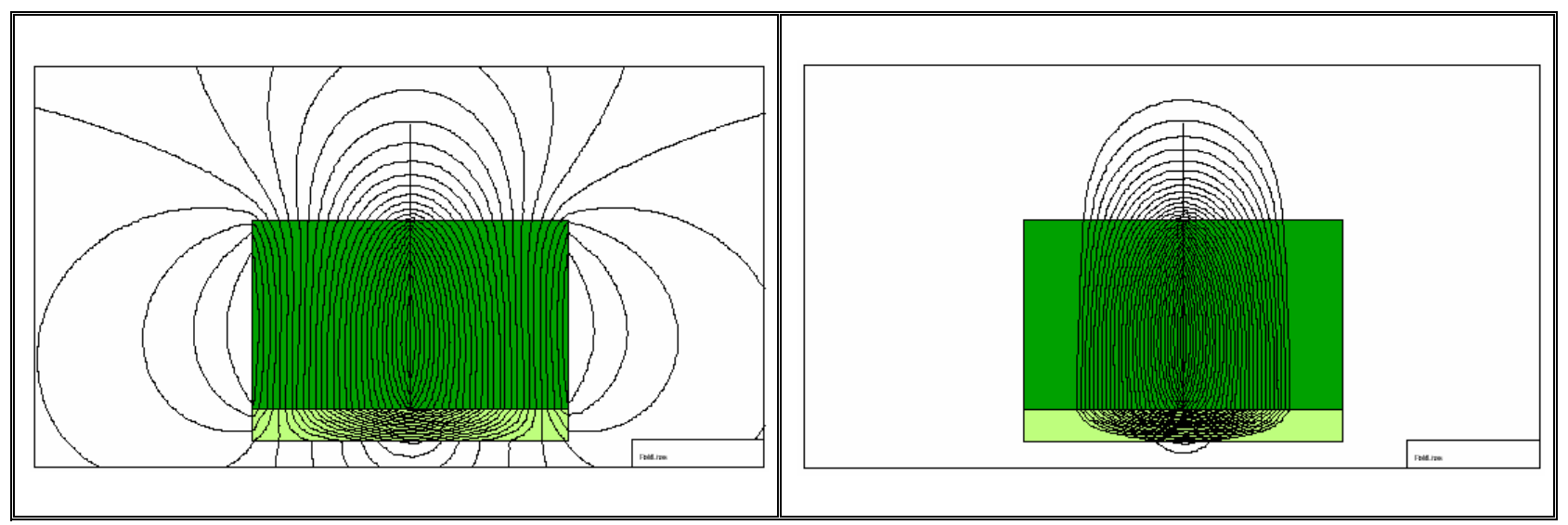

Figure 15. (a) Field lines and (b) field lines (magnified) for concept 4.

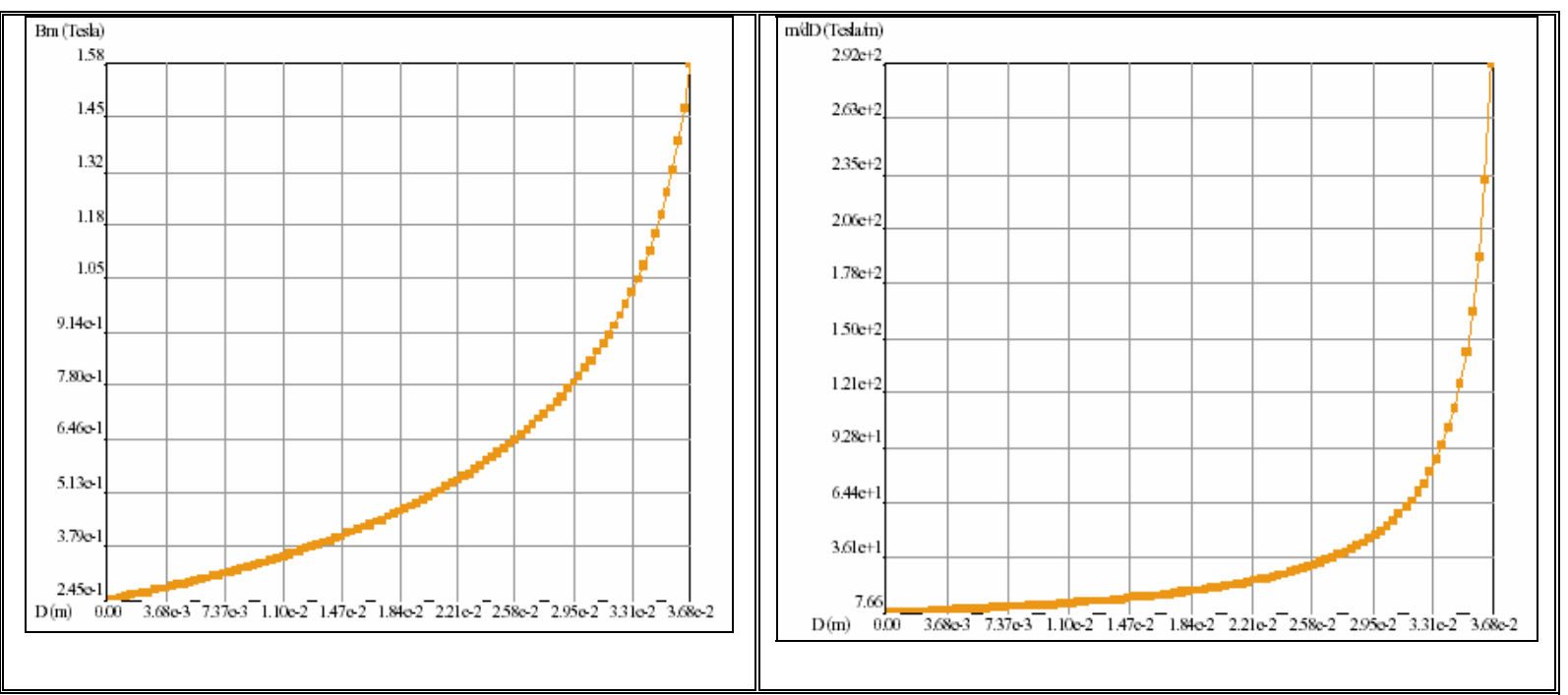

Figure 16. (a) Flux density along centerline and (b) flux density on line (differentiated) for concept 4.

A fifth concept is shown in Figure 17. This concept is that of a pointed magnet or pole piece. The line of interest is again $1.5 \mathrm{in}$. long in this design. The flux density about the line of interest and the gradient about the line of interest are shown in Figure 18. 


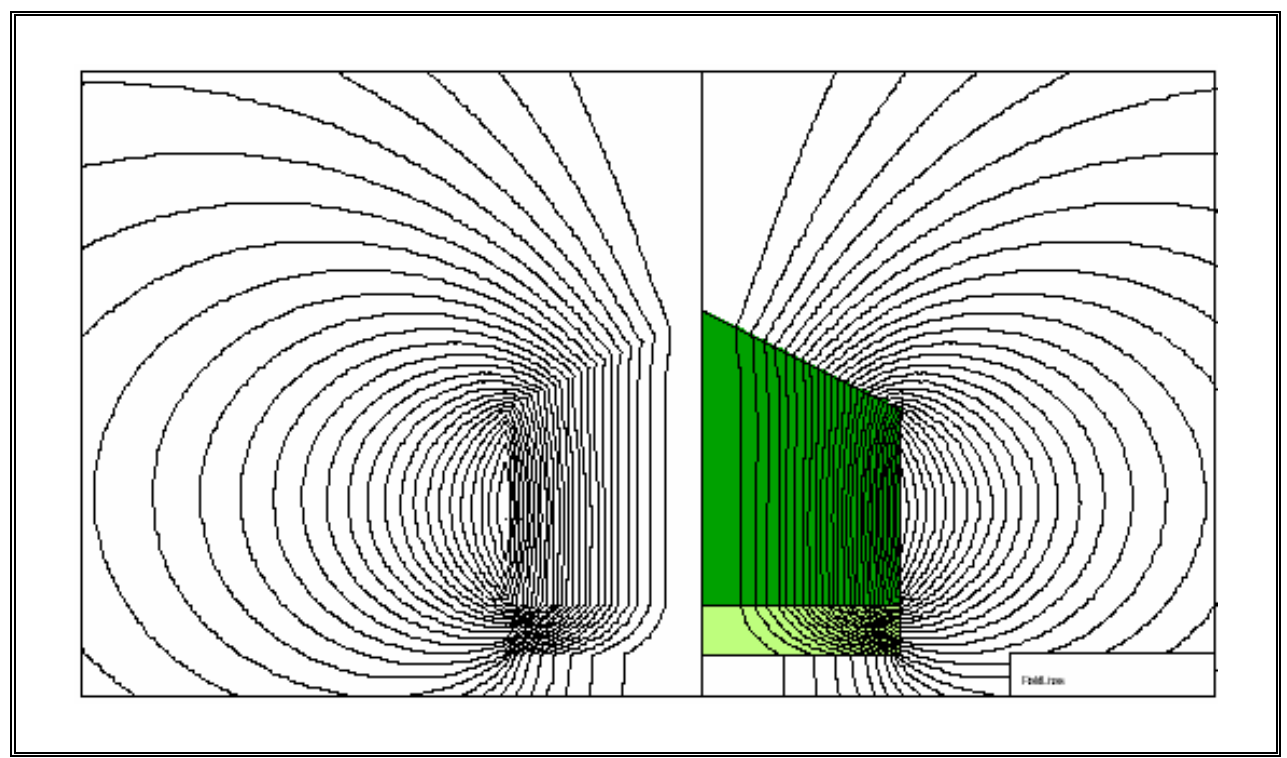

Figure 17. Field lines for concept 5

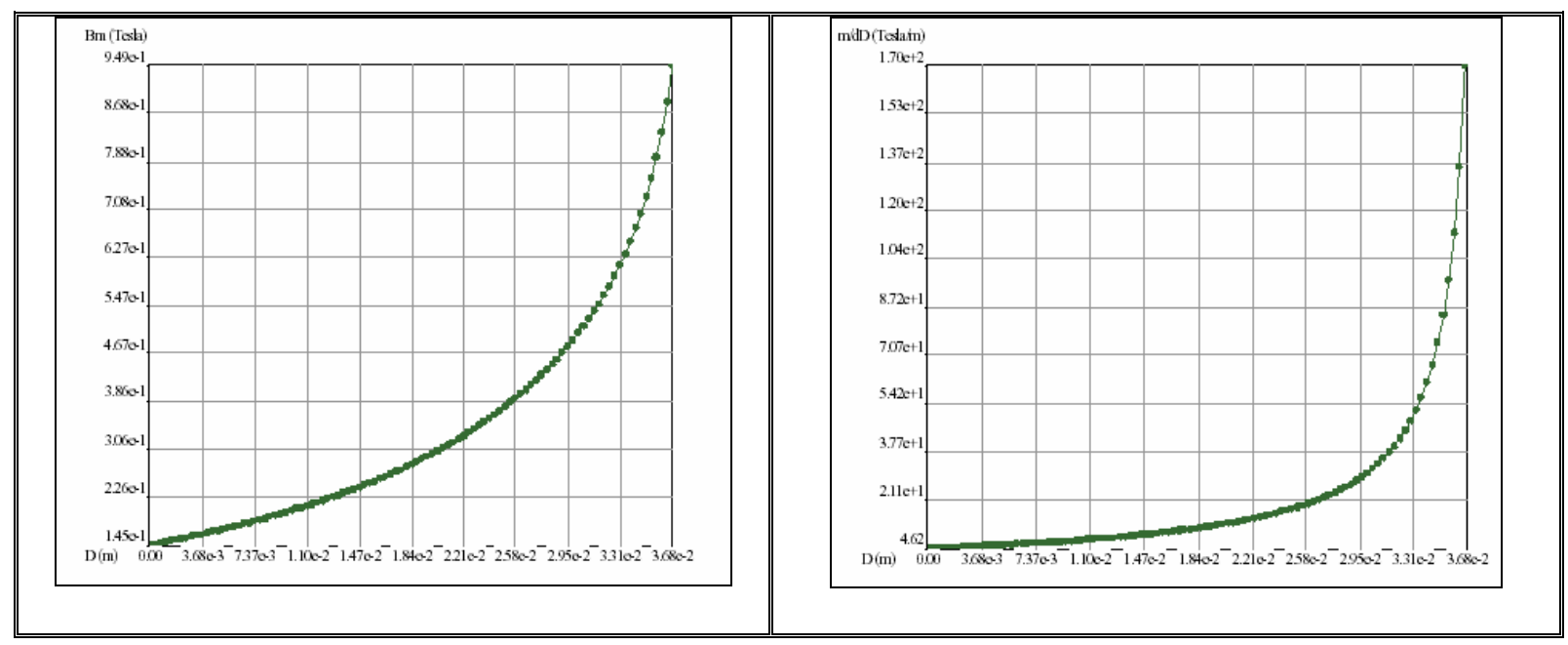

Figure 18. (a) Flux density on line field lines and (b) flux density gradient for concept 5

One thing to keep in mind is that the shape of the field lines does not depict the direction of separation. As expected, the gradient is the governing factor. Consequently, it will be useful to see the flux density profile of configurations 4 and 5 to compare the "sharpness" of the reach. While configuration 4 produces a much broader flux density profile than configuration 5 , it also produces a higher flux density and field gradient, as shown in Figure 19. 


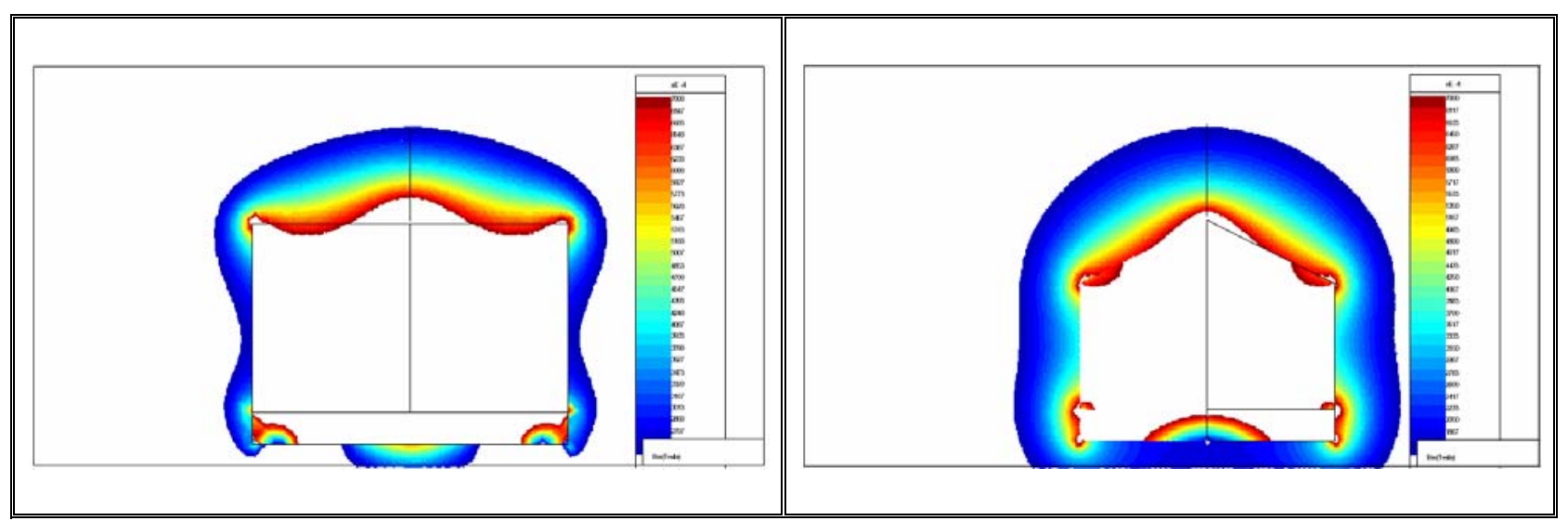

Figure 19. Flux density contours for (a) concept 4 and (b) concept 5 . The graduated legend has units of Tesla and spans from 0.27 to 0.70

\section{SYNTHESIS OF OXIDATION-PROTECTED AND SIZE-CONTROLLED IRON NANOPARTICLES}

The magnetic moments of the polymeric-based micro and nanoparticles are derived completely from the magnetic nanocrystals encapsulated or embedded in the polymer matrix . Since external manipulation of the particles requires high magnetic moments we are pursuing methods of synthesizing materials with higher magnetic moments than those of iron oxides. Attractive options are nickel, cobalt, or iron magnetic nanocrystals. However, a severe limitation in the use of these materials is their instability and tendency to oxidize at room temperature. To overcome oxidation we are investigating the formation of layered nanoparticles containing a core of magnetic material and a thin outer shell that protects the core form oxidation. Using a similar technique, it may be possible to introduce radionuclides into this layered structure, incorporate the magnetic, radioactive nanoparticle into the polymeric matrix for vascular transport, and target tumors.

Iron nanoparticles of controlled size produced by the chemical vapor condensation (CVC) technique are used in this program as precursor material for making biodegradable PLA-based microspheres. Compared with other synthesis techniques, CVC has advantages: it provides control of particle size over the widest possible range and it minimizes agglomeration when particles are dispersed in liquids. The CVC technique can also be used to produce either metallic or oxide magnetic nanoparticles.

The CVC technique involves the decomposition of one or more metal-organic precursor materials in a furnace (Fig. 20). Controlled amounts of precursor vapor are added to a carrier gas, typically nitrogen, by bubbling the carrier gas through a supply of liquid precursor under controlled temperature, pressure, and flow-rate conditions. Temperature-induced decomposition of the precursor results in homogeneous nucleation (condensation) of nanoparticles. Particle size control is attained through adjustment of several process parameters. Particles of sizes from a few nanometers to a few microns in diameter can be produced by CVC, although to date, work in 
this field has mainly focused on making particles as small as possible, typically less than $20 \mathrm{~nm}$ average diameter.
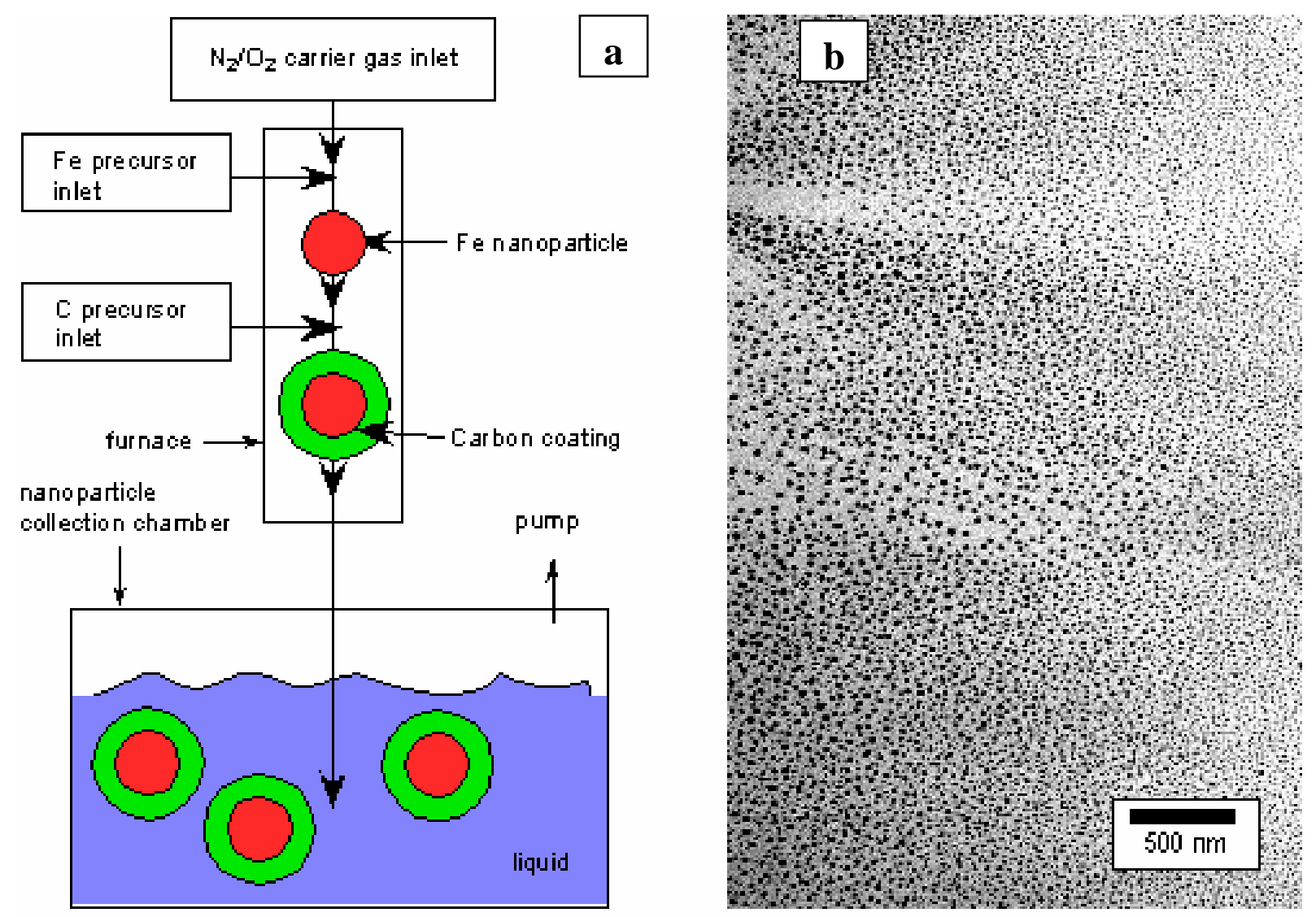

Figure 20. (a) Schematic of chemical vapor condensation (CVC) process for producing sizecontrolled carbon-coated iron nanoparticles dispersed in liquid. (b) Bright-field TEM micrograph of $20 \mathrm{~nm}$ average-diameter iron nanoparticles produced at Argonne National Laboratory by CVC (Bai et al. 2002).

Production of nanoparticles by CVC is a fairly new development, beginning with work on nanometer-sized oxide particles less than 10 years ago (Chang et al. 1995). Other work (Srdic et al. 2001) has demonstrated that CVC can be used to produce coated oxide nanoparticles consisting of a core of one material surrounded by a thin layer of a second material. More recently, it has been demonstrated that $\mathrm{CVC}$ can also be used to produce metallic nanoparticles. In particular, Choi et al. (2001a, 2001b) used CVC to produce iron and cobalt nanoparticles with controlled average sizes from 5 to $18 \mathrm{~nm}$. The magnetic character of their iron particles changed from superparamagnetic to ferromagnetic with increasing size, and the saturation magnetization of iron nanoparticles also increased with increasing size [increasing approximately linearly with particle size from about $10 \mathrm{Am}^{2} / \mathrm{kg}$ at $5 \mathrm{~nm}$ particle diameter to $160 \mathrm{Am}^{2} / \mathrm{kg}$ at $18 \mathrm{~nm}$ particle diameter (Choi et al. 2001a)].

While previous materials produced by CVC were either agglomerated powders (Chang et al. 1995, Choi et al. 2001a, 2001b) or coatings (Srdicet al. 2001), our recent work at Argonne (Bai 
et al. 2002) has shown the potential for synthesizing non-agglomerated nanoparticles dispersed in liquids, such as are desired in the present program. In this case, as shown in Figure 20a, particles are collected downstream of the condensation process by bubbling the flow stream through a liquid (e.g., polyethylene glycol). Non-agglomerated iron nanoparticles with an average size of $20 \mathrm{~nm}$ and a narrow size distribution have been produced using butyl ferrocene as the metal-organic precursor and nitrogen as the carrier gas (Figure 20b).

Experiments to determine the optimal iron nanoparticle size needed for PLA-based sphere production and the conditions needed to achieve this size will be a focus of future studies. We will also determine conditions for coating iron particles with carbon as part of the process for preventing their oxidation and incorporating them into PLA-based spheres. Carbonencapsulation of iron nanoparticles has already been achieved for some processing routes, such as an arc-discharge method (Sun et al. 2000). Carbon-coated metallic nanoparticles have not yet been produced by $\mathrm{CVC}$, but are expected to be achievable using techniques already developed by Hahn and co-workers for making coated oxide nanoparticles (Srdic et al. 2001). For example, we can control the decomposition of the carbon-containing metal-organic precursor to produce excess carbon, or burn all of the iron precursor carbon using controlled additions of oxygen to the flow stream, followed by introduction of a second carbon precursor downstream of the iron precursor in the CVC process.

\section{IN VITRO TESTING FACILITY}

A flow channel apparatus equipped with a microscope-based video that tracks magnetic particle suspension in a controlled environment is shown in Figure 21. The actual prototype for studying the behavior of magnetic particle suspensions under the influence of magnetic fields is shown in Figure 22. Included is a close-up image of the square flow channel ( $2 \mathrm{~mm} \times 2 \mathrm{~mm})$. The design consists of a sonicating bath for mixing the suspension, peristaltic pump for transportation of the fluid from the reservoir tank to the 7-inch-long glass channel ( $2 \mathrm{~mm} \times 2 \mathrm{~mm}$ or $4 \mathrm{~mm} \times 4 \mathrm{~mm}$ ), $\mathrm{NdFeB}$ permanent magnet, digital video and high-resolution camera (microscope), back lighting and video monitor. 


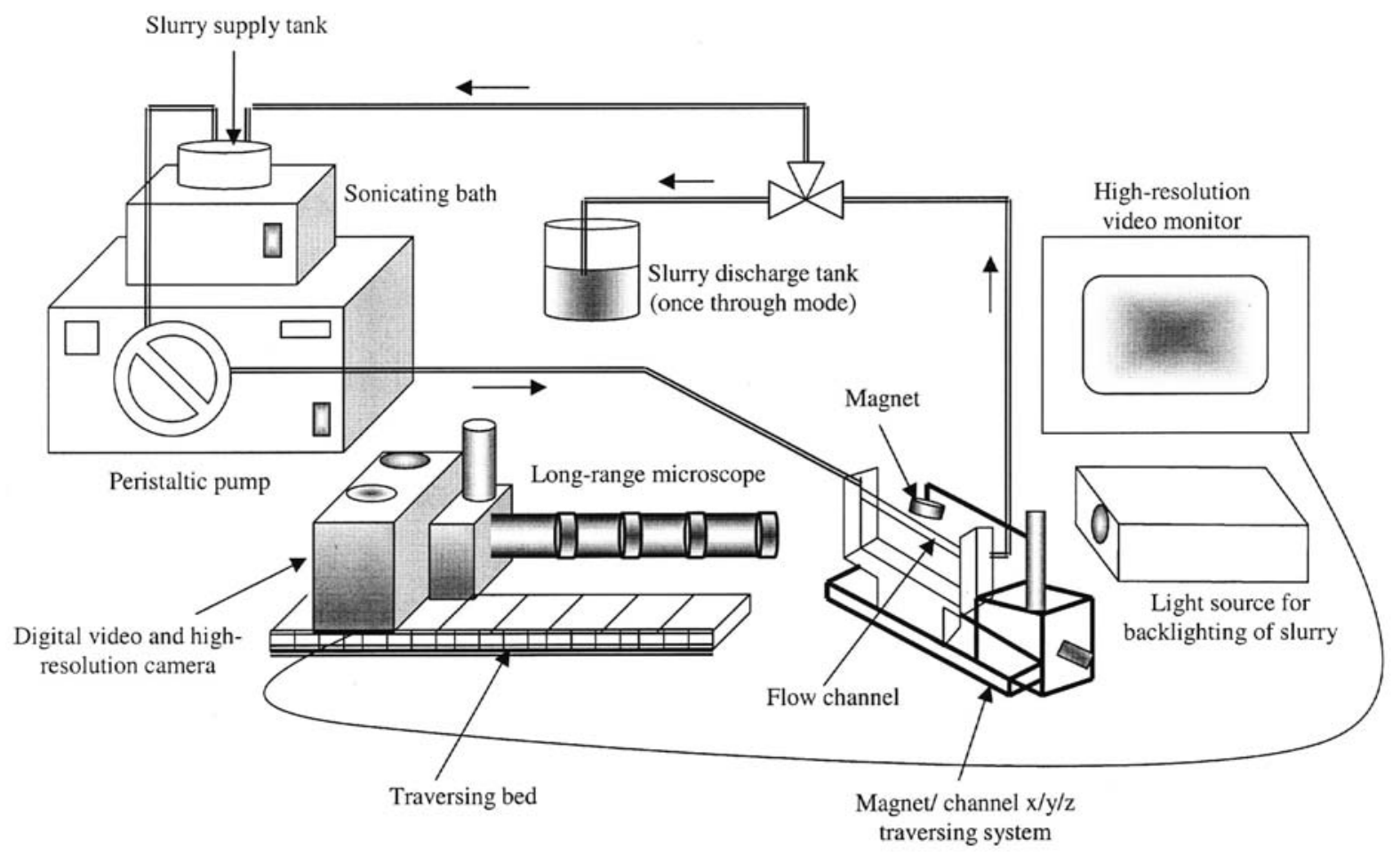

Figure 21. Schematic drawing of apparatus for studying magnetic particle liquid-suspension behavior under influence of magnetic fields.
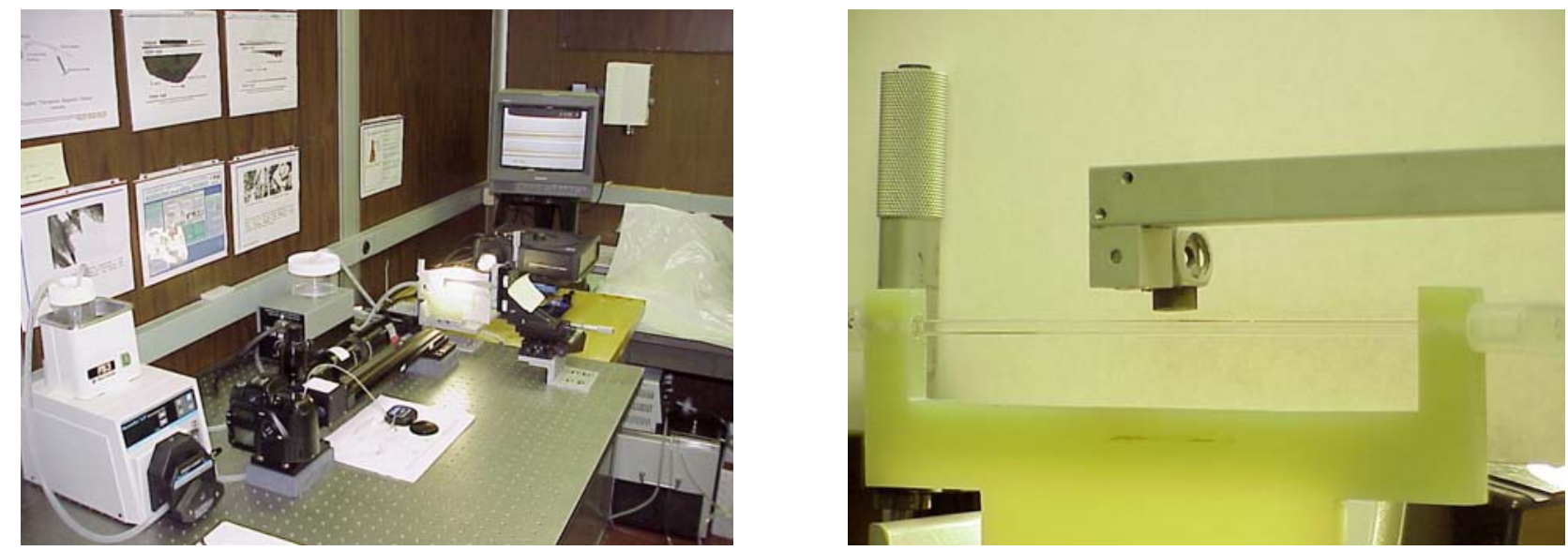

Figure 22. (a) Experimental apparatus for studying magnetic particle liquid-suspension behavior under influence of magnetic fields. (b) Glass, square flow channel (2mm x $2 \mathrm{~mm})$ showing external magnet positioned above the channel.

In a typical experiment, $25 \mathrm{mg}$ of magnetic particles in $200 \mathrm{~mL}$ of distilled water is driven into the flow channel using a peristaltic pump and passed through the glass cell. The magnet is active, permitting immobilization of magnetic particles onto the chamber wall. The supernatant containing the untrapped magnetic particles flowing out through the end of the chamber is recirculated for a specified period of time to compare particles of different magnetophoretic 
mobility (magnetic responsiveness). Similar flow channel experiments were completed with no recirculation (once-through capture), variations in magnetic field and gradient (by displacing the magnet from the channel wall), various channel cross sections and test durations, and various magnetic particle types. The experiments directly highlight the areas of needed optimization (especially inadequate magnetic susceptibilities) and allow for quick optical determination of the behavior of each type of particle in a defined magnetic field and flow velocity.

To date, several conditions have been compared. From the magnetic field plot and by varying the flow velocity, we were able to determine the minimum magnetic attractive forces necessary to retain particles in the target area for one particle type, as shown in Figure 23 (cellulose-based magnetic particles, saturation magnetic susceptibility $\mu_{\text {susc }}=40 \mathrm{emu} / \mathrm{g}$ ), for recirculatory flow where the flow velocity increases with a fixed magnet position. The photos show the dramatic decrease in particle retention as flow increases from arteriole speeds $(8.3 \mathrm{~cm} / \mathrm{s})$ to peak aorta flow $(125 \mathrm{~cm} / \mathrm{s})$ or even to peak flows in (re)stenosed vessels $(208 \mathrm{~cm} / \mathrm{s})$.

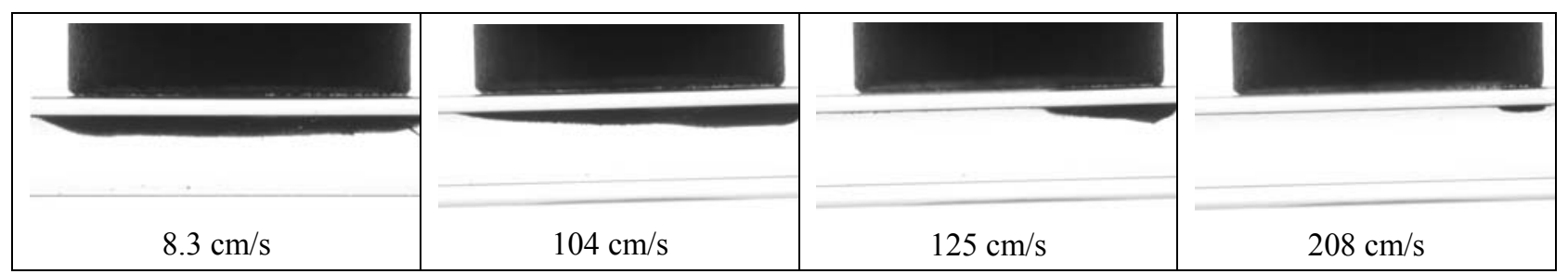

Figure 23. Magnetic particles held under magnet after 10 minutes of flow circulation. The field strength applied to the particles at the centerline of the magnet at the flow channel wall is 13 $\mathrm{T}^{2} / \mathrm{m}$ (magnetic field $=0.4-\mathrm{T}$ at $\mathrm{NdFeB}$ magnet surface).

Figure 24 shows, in the same flow channel setup at $42 \mathrm{~cm} / \mathrm{s}$, how particles of different magnetic susceptibility behave. The silica-based Sicastar-M particles with a saturation magnetic susceptibility of $6 \mathrm{emu} / \mathrm{g}$ were clearly much less attracted and captured by the magnet than the Magacell-PEG-5000 particles of $40 \mathrm{emu} / \mathrm{g}$. The particle concentrations at the magnet edges are a result of the relatively large spatial gradients in magnetic field density at these locations.

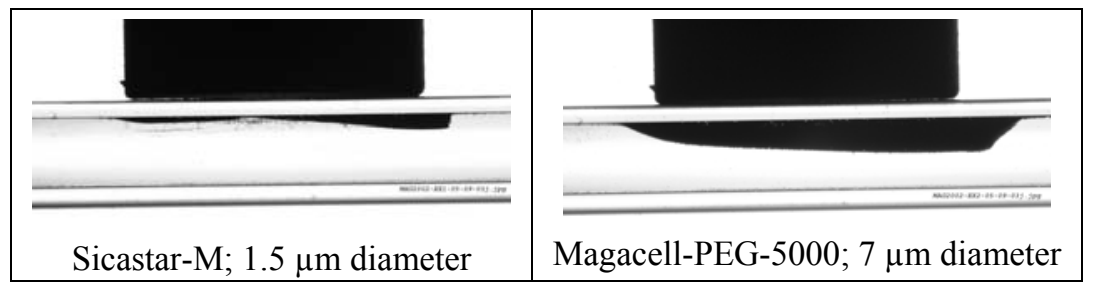

Figure 24. Comparison of particles of different magnetophoretic mobility (magnetic responsiveness). The $25 \mathrm{mg}$ magnetic particles in $200 \mathrm{~mL}$ of water were recirculated for 10 minutes, using the setup from Figure 22.

An entire set of experiments was completed in the manner described. The accumulated pile of magnetic particles was quantified by analysis of digital photos for these experiments. The results are used as benchmark input into the numerical models being developed to predict the two-phase 
flow behavior of the magnetic particles in the presence of well-characterized magnetic field profiles (see following section). In the future, the in vitro testing facility will be expanded to include bifurcations, rounded cells, various cell diameters, and liquids. The resulting data on the accumulation of magnetic particles will be used to test the model.

\section{NUMERICAL MODELING OF MAGNETIC PARTICLES}

\section{a. Background}

Modeling of magnetic particle therapy involves interaction among electromagnetic fields, fluid mechanics, and therapeutic phenomena. Modeling is needed to help optimize the performance of targeted magnetic particle delivery, enhance its therapeutic value, and expedite medical application. A model incorporating computational fluid dynamics (CFD) and experimental magnetic field data has been developed to predict the magnetic particle behavior in a $2 \mathrm{~mm} \times 2$ $\mathrm{mm}$ test section at different flow rates. The modeling effort has two objectives. In the near term, we seek to validate modeling assumptions by comparison with laboratory experiments. Key aspects of this phase are that the geometries are simple and readily meshed, and that the particle trapping behavior is well characterized by experiments. The longer-term objective is to model particle migration in specific vascular geometries under realistic (pulsatile) flow conditions. The purpose is to provide guidelines (injection points, magnet locations, strengths, switching times, etc.) that optimize the delivery of magnetic particles in suspension to targets for various medical treatment scenarios.

This section presents initial computations of particle deposition rates and distributions to match the flow experiments performed in the in vitro testing facility. The simulations are predicated on the following modeling assumptions:

- The suspension is sufficiently dilute that particle-particle interaction can be neglected.

- The suspension is sufficiently dilute that particle-fluid interaction can be neglected - that is, particles in suspension do not influence the fluid dynamics.

- Particle build-up and subsequent channel blockage are neglected.

- On entering the channel test section, the particles are uniformly (randomly) distributed in space.

- The particles are sufficiently small that the Stokes drag law is appropriate for computing the fluid forces acting on the particles.

- The flow field is steady, spatially fully developed, and laminar.

- Radial $B$-field gradients are neglected.

- The particle magnetism is in a fully saturated state. 
- The particle diameter is $D_{P}=7 \mu \mathrm{m}$, although, in fact, the particle diameters are known to have a Gaussian-like distribution centered roughly at $7 \mu \mathrm{m}$.

The assumptions of dilute suspensions and steady flow conditions allow us to decouple the fluid velocity field and particle tracking computations. Particle positions are computed using $F=m_{e} \mathrm{a}$, where a is the particle acceleration, $m_{e}$, is the effective mass (accounting for fluid-inertia effects), and $F$ is the net force on the particle computed as

$$
\mathrm{F}=\mathrm{F}_{f}+\mathrm{F}_{\mathrm{m}} .
$$

The modeling assumptions for the computation of the fluid and magnetic forces, $F_{f}$ and $F_{M}$, respectively, are discussed below.

\section{Experimental Configuration}

The experimental flow rig is a closed-loop circulation system with a suspension slurry comprising $200 \mathrm{~mL}$ of deionized water charged with $1.6 \times 10^{6}(25 \mathrm{mg})$ Magacell-PEG-5000 particles (Cortex Biochem, Inc., San Leandro, California). The mean particle diameter is $7 \mu \mathrm{m}$. The test section is a $2 \mathrm{~mm} \times 2 \mathrm{~mm}$ square channel approximately $25 \mathrm{~cm}$ in length, with a 9-mmdiameter magnet positioned above the channel. The channel wall thickness is $0.43 \mathrm{~mm}$, and the surface of the magnet is roughly $0.52 \mathrm{~mm}$ above the upper surface of the channel interior. The corresponding model configuration is illustrated in Figure 25. We assume that the origin of coordinate system is centered in the channel, just below the magnet, with the flow traveling in the positive $x$ direction, and the lower surface of the magnet located at $y=1.52 \mathrm{~mm}$. 

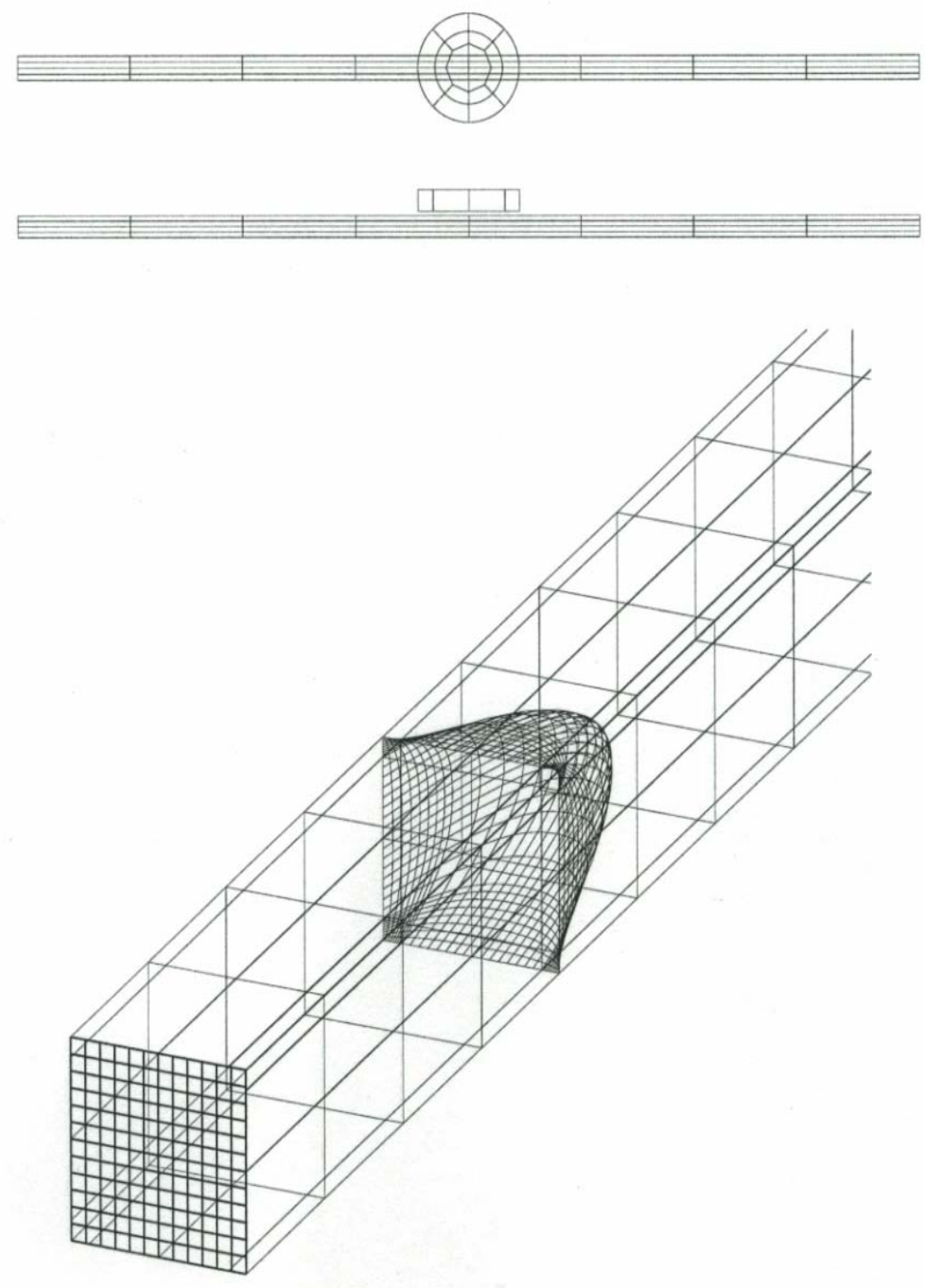

Figure 25. Model configuration. (Top) plan and side views of channel-magnet orientation, (bottom) oblique view of particle inlet cells and computed axial velocity profile.

\section{Fluid Dynamics of the Channel}

The baseline flow rate through the channel is $100 \mathrm{~mL} / \mathrm{min}$. Computations are also performed at $50 \mathrm{~mL} / \mathrm{min}$ and $200 \mathrm{~mL} / \mathrm{min}$. At $200 \mathrm{~mL} / \mathrm{min}$, the channel flow Reynolds number is

$$
\operatorname{Re}_{H}=\frac{H \bar{V}}{v}=\frac{(0.002 \mathrm{~m})(0.8333 \mathrm{~m} / \mathrm{s})}{\left(1 \times 0^{-6} \mathrm{~m}^{2} / \mathrm{s}\right)}=1667
$$

Since transition to turbulence occurs for $\mathrm{Re}>2000$, the steady laminar flow assumption is justified for all three flow rates. Here, $H$ is the channel height, $\bar{V}$ the mean flow speed, and $v$ the kinematic viscosity. An approximate development length, estimated from boundary layer theory, is 


$$
L_{\mathrm{Dev}} \approx \frac{\sqrt{R e_{H}}}{5} \frac{H}{2} \approx 4 H,
$$

which indicates that the fluid velocity profile will be unchanging for any $\chi>\chi_{0}+4 H$, where $\chi_{0}$ is the location of the channel entrance. In this initial set of computations, we have $\chi_{0}=-40 \mathrm{~mm}$. The exit is located at $\chi_{1}=+40 \mathrm{~mm}$.

Because of the lack of particle feedback on the flow field, it is possible to compute the fluid velocity distribution a priori, and use this as input to an independent particle tracking program. The flow field was computed using spectral elements of order $N=5$ with the mesh shown in the lower part of Figure 25, which also shows the velocity profile at a cross section $8 H$ downstream of the channel entrance. This is a straightforward flow simulation and, under the stated assumptions, the flow profiles may be considered exact.

\section{Magnetic Field Conditions}

The magnet used in the experiments is a 9-mm-diameter $\mathrm{NdFeB}$ magnet with $B$ field characteristics (Figure 26). Since the orientation of the magnet is unknown, the data were symmetrized for the numerical computations by summing the original $B$-field profile with its reflection about the centerline. A bicubic spline was then passed through the data, which were differentiated and smoothed to obtain the normal component of the $B$-field gradient, $d B / d y$, required for the force computation.

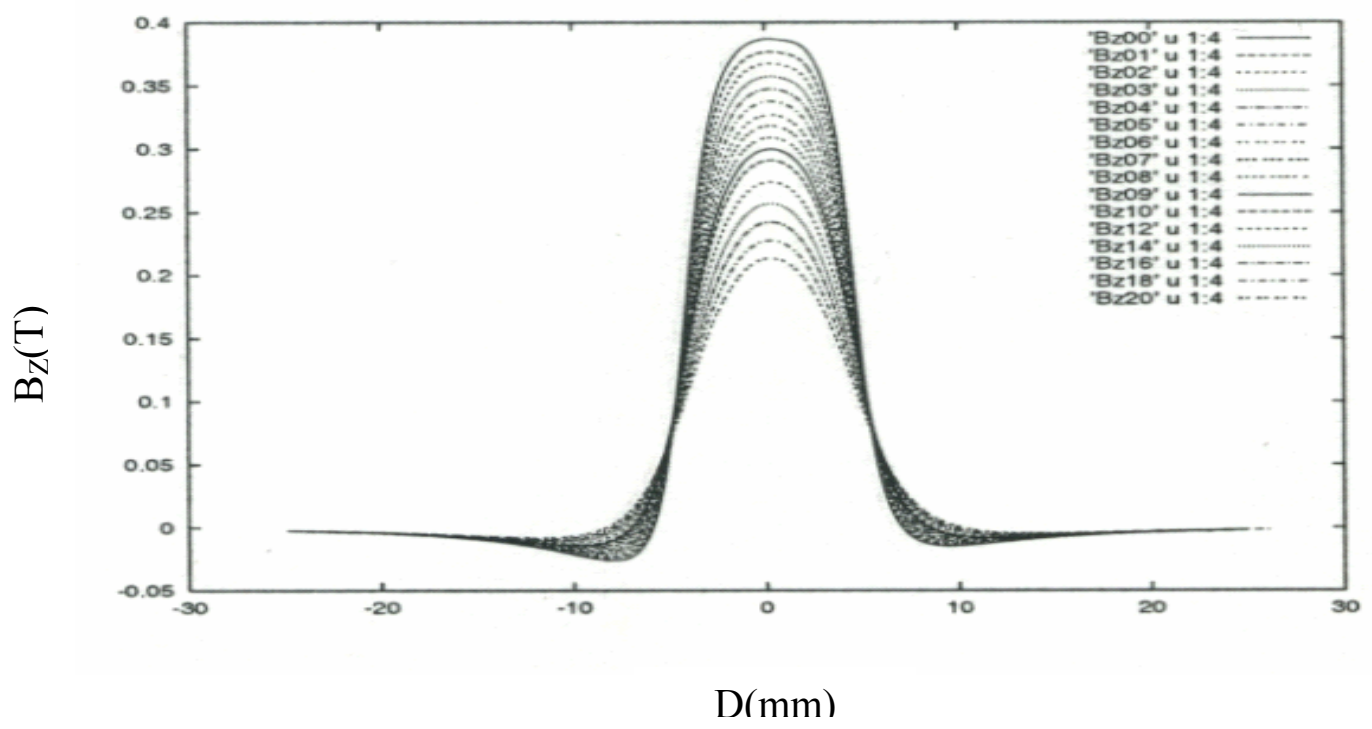

Figure 26. Measured B-field distribution.

\section{Particle Characteristics and Magnetic Force}

The magnetic force was computed based on known properties of the Magacell-PEG-5000 particles. These are particles with a distribution of diameters centered roughly at $D_{p}=7 \mu \mathrm{m}$, which was chosen as the diameter for all of the numerical simulations. The particles are $50 \mathrm{wt} \%$ magnetite with a cellulose binder. Details of the particle composition are given below. 


$$
\begin{array}{ll}
\rho_{\mathrm{p}} & \left.=2,400 \mathrm{~kg} / \mathrm{m}^{3} \text { (magnetite }+ \text { binder }\right) \\
D_{P} & =7 \times 10^{-6} \mathrm{~m} \\
M s_{p} & =40 \mathrm{emu} / \mathrm{g} \text { particle }=80 \mathrm{emu} / \mathrm{g} \text { magnetite } \\
\mathrm{m}_{p} & =\left(\rho_{p} * \pi * \mathrm{D}_{p}^{3}\right) / 6=4.31 \times 10^{-13} \mathrm{~kg}
\end{array}
$$

Properties of magnetite are:

$$
\begin{array}{ll}
\rho_{m} & =5,150 \mathrm{~kg} / \mathrm{m}^{3} \\
\mathrm{Ms}_{\mathrm{m}} & =(80 \mathrm{emu} / \mathrm{gm})\left(5.15 \mathrm{gm} / \mathrm{cm}^{3}\right)=412 \mathrm{emu} / \mathrm{cm}^{3} \\
\mathrm{~m}_{m} & =\mathrm{m}_{p} / 2=2.15 \times 10^{-10} \mathrm{~g}=2.15 \times 10^{-13} \mathrm{~kg} \\
V_{m} & =\mathrm{m}_{m} / \rho_{M}=4.18 \times 10^{-17} \mathrm{~m}^{3}
\end{array}
$$

Combining these data with the conversion constants $1 / 1000 \mathrm{~A} / \mathrm{m}=$ Gauss and 1 Tesla $\equiv 1 \mathrm{~N} \cdot \mathrm{m} / \mathrm{A}$ (or $1 \mathrm{~T}=10000$ Gauss), we obtain the following estimate for $F_{M}$ :

$$
\begin{aligned}
F_{m} & =\mathrm{V}_{\mathrm{m}} \mathrm{M}_{\mathrm{S}_{\mathrm{m}}} \frac{\mathrm{dB}}{\mathrm{dy}} \\
& =4.18 \times 10^{-17} \mathrm{~m}^{5} \cdot 4.12 \times 10^{-5} \mathrm{~A} / \mathrm{m} \frac{\mathrm{dB}}{\mathrm{dy}} \\
& =1.72 \times 10^{-11} \mathrm{~N} \cdot \mathrm{m} / \mathrm{T} \frac{\mathrm{dB}}{\mathrm{dy}} .
\end{aligned}
$$

Note, to account for the change in the magnetic field gradient and consequent change in the particle magnetization state, we must take the absolute value of $\frac{d B}{d y}$ (the particle is always pulled toward the magnet). Thus, the magnetic force on the particle is

$$
\mathbf{F}_{\mathrm{m}}=\mathrm{C}_{\mathrm{m}}\left|\frac{\mathrm{dB}}{\mathrm{dy}}\right| \hat{j}
$$

where $C_{m}=1.72 \times 10^{-11} \mathrm{~N} \cdot \mathrm{m} / \mathrm{T} ; \frac{d B}{d y}$ is the vertical component of the magnetic field gradient at the particle location; and $\hat{j}$ is the unit vector in the $y$ direction. As a point of reference, we note 
that the magnetic force at the magnet centerline $(x=0, z=0)$ ranges from roughly $1 \times 10^{-9} \mathrm{~N}$ to $1.7 \times 10^{-9} \mathrm{~N}$ as $y$ varies from $-0.001 \mathrm{~m}$ to $+0.001 \mathrm{~m}$.

\section{Dynamics of the Particle-Fluid Interaction}

The fluid forces on the particle are well-characterized by the Stokes drag formula:

$$
\mathbf{F}_{f}=3 \pi \mu \mathrm{D}_{\mathrm{p}}\left(\mathbf{V}_{\mathrm{f}}-\mathbf{v}_{\mathrm{p}}\right)
$$

where $\mu=10^{-3} \mathrm{~kg} / \mathrm{ms}$ is the dynamic viscosity of water. Stokes drag is applicable provided the (relative) particle Reynolds number is lower than $\mathrm{Re}_{\mathrm{p}} \sim 1$. Assuming that the particle is not moving $\left(\mathbf{V}_{\mathrm{p}}=0\right)$, one finds $R e_{p}=\mathrm{D}_{\mathrm{p}} \overline{\mathrm{V}} / v \approx 6$. Generally, we will have $\left|\mathrm{V}_{\mathrm{f}}-\mathrm{V}_{\mathrm{p}}\right|$ « $\overline{\mathrm{V}}$ and can therefore expect that $\operatorname{Re}_{\mathrm{p}} \ll 1$. The Stokes drag formula (Equation 5) is thus a reasonable assumption.

The other fluid dynamic phenomenon that we account for is the added mass that is associated with fluid accelerations induced by particle acceleration. If a particle is suddenly accelerated from rest, it must also accelerate the fluid that it displaces and one consequently observes additional inertia. Basic fluid dynamics calculations show the apparent increase in inertia to be half of the mass of the displaced fluid. Thus, the equivalent mass is given by

$$
\mathrm{m}_{\mathrm{e}}=\mathrm{m}_{\mathrm{p}}+\frac{1}{2} \mathrm{pfV}_{\mathrm{p}}=4.13 \times 10^{-13}+\frac{1}{2} \cdot 1000 \cdot 8.36 \times 10^{-17}=4.55 \times 10^{-13} \mathrm{~kg},
$$

which is the value used in all computations of particle acceleration.

\section{b. Computation of Particle Trajectories}

The particle trajectories $\mathbf{X}_{p}(t)$ are described by the first-order ordinary differential equation

$$
\frac{\mathrm{d}}{\mathrm{dt}}\left(\begin{array}{l}
\mathbf{X}_{\mathrm{p}} \\
\mathbf{V}_{\mathrm{p}}
\end{array}\right)=\left(\begin{array}{cc}
0 & 1 \\
0 & -\mathrm{C}_{\mathrm{f}}
\end{array}\right)\left(\begin{array}{l}
\mathbf{X}_{\mathrm{p}} \\
\mathbf{V}_{\mathrm{p}}
\end{array}\right)+\left(\begin{array}{c}
0 \\
\mathrm{C}_{\mathrm{M}} \frac{\mathrm{dB}}{\mathrm{dy}} \hat{\mathrm{j}}+\mathrm{C}_{\mathrm{f}} \mathbf{V}_{\mathrm{f}}
\end{array}\right)
$$

Here,

$$
C_{f}=3 p \mu D_{p} / m_{e}
$$

is a constant characterizing the acceleration due to the Stokes drag. Equation 7 is a system of six equations - three positions and three velocities. Initially, we used an adaptive 4th-order Runge Kutta (RK4) scheme to integrate Equation 7 in time. However, it was found that the explicit RK4 scheme required thousands of steps to compute the particle motion, despite the fact that the trajectories were smooth. The small time step size is attributable to the stiffness of the system (Equation 7), which we now examine. 
In the channel, Equation 7 essentially decouples in each direction because the fluid flows only in the $x$ direction, the magnetic force acts only in the $y$ direction, and there is no displacement in the $z$ direction. It is instructive to consider the motion of a particle initially at rest at the bottom of the channel, $\mathbf{X}_{p}=(0,-0.001,0)$, in a stationary flow field $\left(\mathbf{V}_{f}=0\right)$. Since the only motion will be in the $y$ direction, Equation 7 reduces to a system of two equations:

$$
\frac{d}{d t}\left(\begin{array}{c}
y_{p} \\
v_{p}
\end{array}\right)=\left(\begin{array}{cc}
0 & 1 \\
0 & -C_{f}
\end{array}\right)\left(\begin{array}{c}
y_{p} \\
v_{p}
\end{array}\right)+\left(\begin{array}{c}
0 \\
C_{M} \frac{d B}{d y}
\end{array}\right) .
$$

Note that this system is identical to the $y$-component of Equation 7 and differs only by the fact that $\frac{d B}{d y}$ will not vary due to displacement in the $x$ direction.

We non-dimensionalize Equation 8 through the introduction of new variables,

$$
\widetilde{y}=\frac{y_{p}}{H}, \quad \widetilde{v}=\frac{v_{p}}{H / t_{m}}, \quad \widetilde{t}=\frac{t}{t_{m}}
$$

where the characteristic timescale

$$
\mathrm{t}_{\mathrm{m}}=\left(\frac{\mathrm{Hm}_{\mathrm{e}}}{\max \left|F_{m}\right|}\right)^{1 / 2}
$$

To within a factor of $\sqrt{2}, \mathrm{t}_{\mathrm{m}}=7.3 \times 10^{-4} \mathrm{~s}$ is the time required for a particle initially at rest to traverse the channel under the influence of the magnetic field alone, assuming that the force is at its maximum throughout the domain and that the fluid resistance is zero. Substituting Equation 9 into Equation 8, we obtain

$$
\frac{d}{d \tilde{t}}\left(\begin{array}{l}
\tilde{y} \\
\tilde{v}
\end{array}\right)=\left(\begin{array}{cc}
0 & 1 \\
0 & -\widetilde{\mathrm{C}}_{\mathrm{f}}
\end{array}\right)\left(\begin{array}{l}
\tilde{y} \\
\tilde{v}
\end{array}\right)+\left(\begin{array}{l}
0 \\
1
\end{array}\right)
$$

Here, we have also substituted $\max \left|\mathbf{F}_{\mathrm{m}}\right|$ for $\mathrm{C}_{\mathrm{m}} \frac{\mathrm{dB}}{\mathrm{dy}}$. With these assumptions, there is a single non-dimensional constant characterizing the behavior of Equation 10, namely

$$
\widetilde{\mathrm{C}}_{\mathrm{f}}=\frac{\mathrm{t}^{2}{ }_{\mathrm{m}}}{\mathrm{H}} \mathrm{C}_{\mathrm{f}}=\frac{3 \pi \mu \mathrm{D}_{\mathrm{p}}}{\max _{\mathrm{m}}} \approx 38.8
$$


$\widetilde{C}_{f}$ is the damping factor that drives the particle acceleration to zero. The particle attains its terminal velocity, in which the magnetic force is balanced by viscosity, on a timescale that is roughly 40 times shorter than the free-fall timescale. This scale disparity suggests that the acceleration, $\frac{d \mathbf{V}_{\mathrm{p}}}{\mathrm{dt}}$, should be treated implicitly. In fact, a reasonable assumption would be to replace the dynamical equation

$$
\mathrm{m}_{\mathrm{e}} \frac{\mathrm{d} \mathbf{V}_{\mathrm{p}}}{\mathrm{dt}}=\mathrm{C}_{\mathrm{m}}\left(\mathbf{V}_{\mathrm{f}}-\mathbf{V}_{\mathrm{p}}\right)+F_{m}
$$

with the kinematic relation

$$
\mathbf{V}_{\mathrm{p}}=\mathbf{V}_{\mathrm{f}}+\frac{1}{\mathrm{C}_{m}} F_{m}
$$

Although the validity of this assumption needs to be checked, it has the potential of greatly simplifying magnetic particle modeling, particularly under unsteady flow conditions.

\section{c. Computation of Particle Trapping}

Initial trapping rates were estimated by computing particle trajectories in a steady state, fully developed, base-flow configuration. The base flow was computed in a three-dimensional channel $0.08 \mathrm{~m}$ long with a $2 \mathrm{~mm} \times 2 \mathrm{~mm}$ square cross section. The spectral element algorithm described in Equation 5 was used to solve the incompressible Navier-Stokes equations in the flow domain. Periodic boundary conditions were used in the axial flow direction and unit forcing (mean pressure gradient) was specified to establish the flow through the channel. At the Reynolds numbers of interest, the flow is laminar and the velocity profiles are similar for all cases. Thus, the results of a single computation can be scaled to obtain the velocity field for each desired flow rate.

The captured particle flux was determined by identifying the region in the channel cross section that delineates trapped particles as well as the mean particle flux entering the trapping region. The trapping region was determined by laying out an $m \times m$ grid of squares at a cross section $30 \mathrm{~mm}$ upstream of the magnet center and releasing a single particle from the center of each square. The particles were tracked using the Crank-Nicolson/Adams-Bashforth scheme and were deemed to be "trapped" if they came within one particle radii of the upper wall. Figure 27a shows contours of axial velocity at intervals corresponding to $0.1 k \mathrm{U}_{\max }$ where $k=1,2,3, \ldots, 9$. Figures $27 \mathrm{~b}-27 \mathrm{~d}$ show the region (identified by circles) corresponding to particles that are trapped by the magnet at flow rates of 50,100 , and $200 \mathrm{~mL} / \mathrm{min}$, respectively.

The fractional capture rate is computed as follows. We assume that the dilute particle slurry upstream of the magnet contains a uniform distribution of particles (in $\mathrm{R}^{3}$ ), with particle density $\rho_{p}$, measured in particles $/ \mathrm{m}^{3}$, which is a function of time and the mixing assumptions discussed below. The net particle flux through each of the $\mathrm{m}^{2}$ cells is thus 


$$
\rho_{\mathrm{p}} \int_{\text {cell }} \mathrm{wdA} \approx \rho_{\mathrm{p}} \mathrm{w}_{\text {cell }} \mathrm{A}_{\text {cell }},
$$

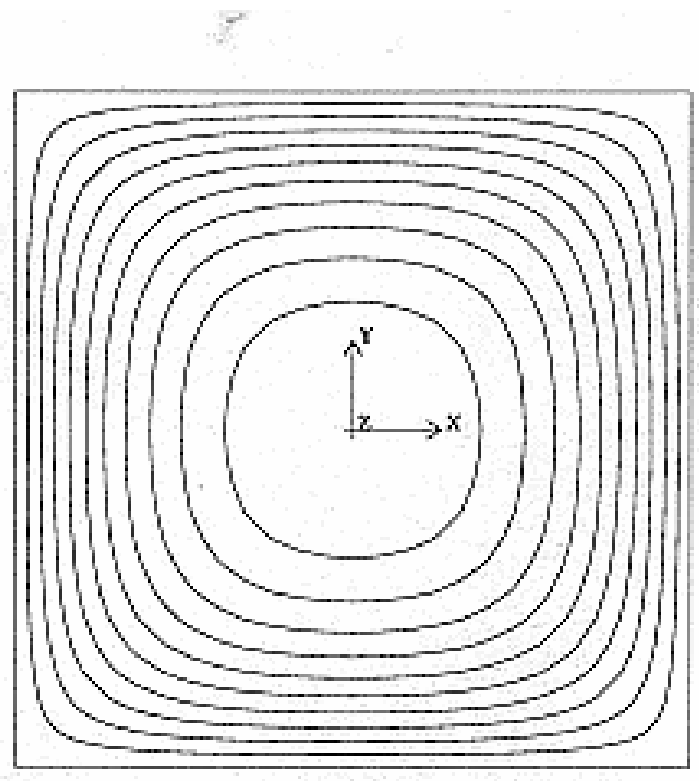

(a)

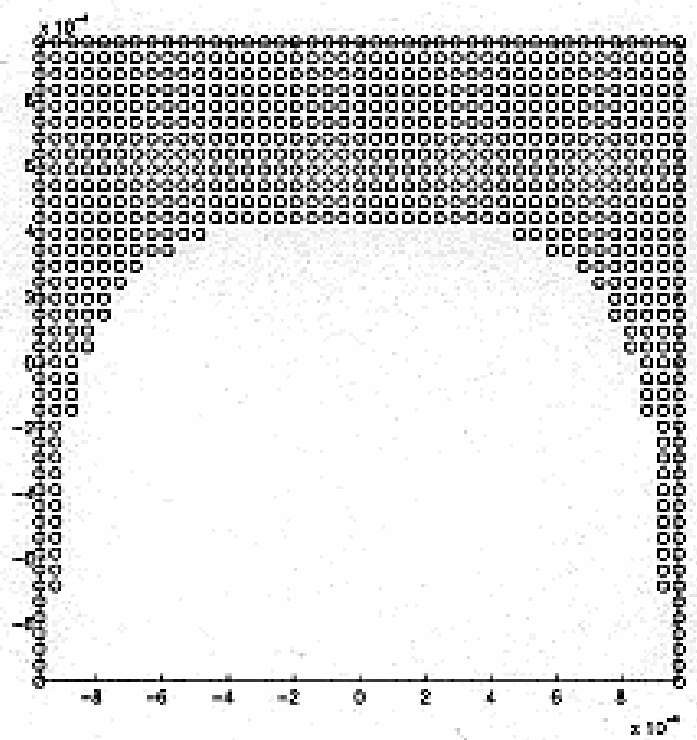

(c)

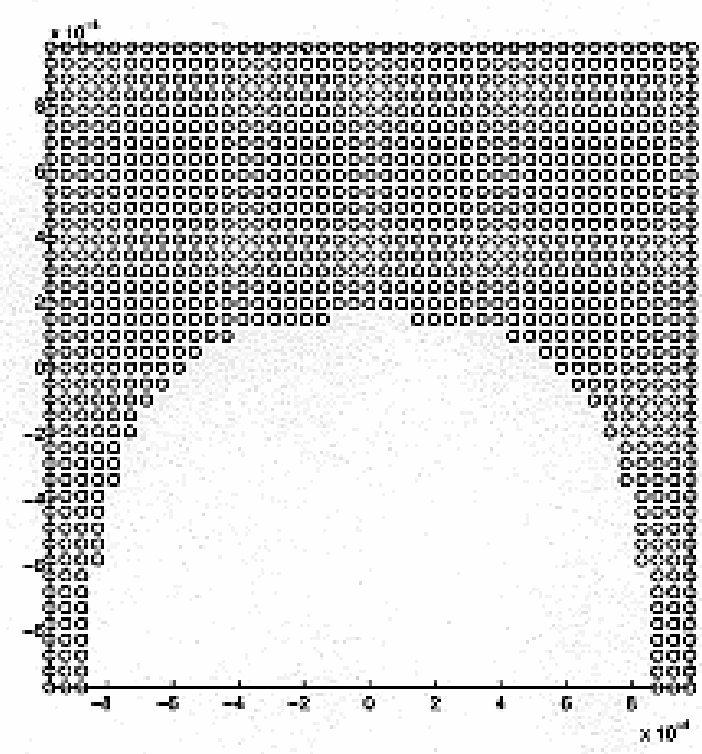

(b)

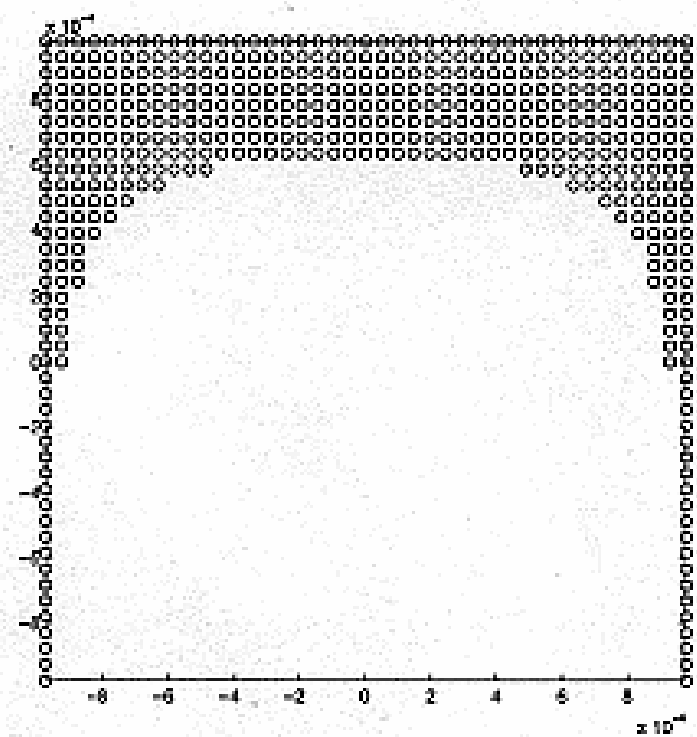

(d)

Figure 27. Velocity contours (a) and particle capture zones, indicated by circles, at (b) 50(c) $100-$, and (d) $200-\mathrm{mL} / \mathrm{min}$ flow rates. The magnet is positioned at the top-center of each plot. The blank regions in (b) - (d) indicate regions of no particle capture.

where w is the axial velocity component, $\mathrm{w}_{\text {cell }}$ is the velocity at the cell center, and $A_{\text {cell }}$ is the cross-sectional area of the cell. The total particle capture rate is computed by summing over all 
the cells. Values of the computed capture fraction $(1=$ all particles captures, $0=$ no particles capture) are plotted in Figure 28.

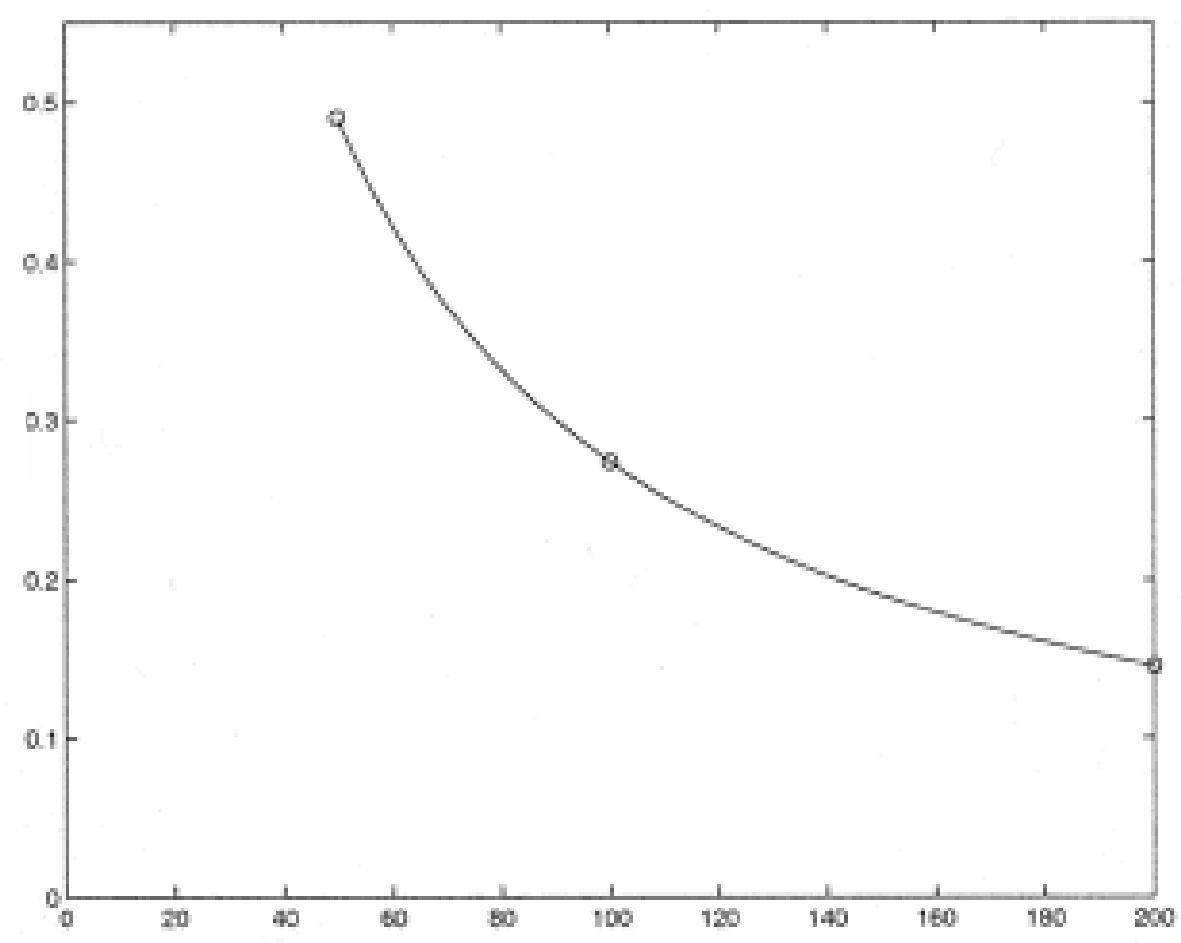

Figure 28. Predicted capture fractions (y-axis) for 7- $\mu \mathrm{m}$ particles at 50-, 100-, and 200-mL/min flow rates (x-axis), with exponential fit (line).

\section{d. Particle Accumulation Rate}

We assume that a fraction, $f_{t} \in[0,1]$, is being trapped by the magnet as the particle slurry flows through the channel past the magnet. If the upstream particle density is $\rho_{p, u}$ the particle density downstream of the magnet is given by

$$
\rho_{p, d}=\left(1-f_{\mathrm{t}}\right) \rho_{p, u}
$$

Note that $f_{\mathrm{t}}$ can be a function of time, according to the local flow conditions in the vicinity of the magnet. However, we presently assume that it is constant and defer the issue of pile buildup and flow-field modifications to $f_{\mathrm{t}}$ to a later report. To estimate the particle accumulation rate, we consider two scenarios that represent the extremes of possible mixing conditions. In the first, we assume that the volume of the mixer in the flow loop is very small compared with the total flowloop volume and that axial mixing can be neglected. Under these conditions, the fraction of trapped particles per total fluid volume exchange is simply $f_{\mathrm{t}}$. After $k$ passes through the system, the number of particles remaining in suspension is given by

$$
N_{p, k}=N_{P, 0}\left(1-f_{\mathrm{t}}\right)^{k} \text {. }
$$


Denoting time to pass the total fluid volume through the flow loop as $t_{i}$, we can rewrite Equation 14 as a function of time:

$$
N_{p}(t)=N_{p,}(0)\left(1-f_{t}\right)^{\frac{t}{t_{i}}}
$$

where it is understood that Equation 15 should be evaluated only at discrete time points $t=k t_{i}$. The second mixing scenario assumes that the mixer volume is essentially equal to the flow loop volume and that any depletion of particles in the magnet vicinity is immediately sensed throughout the flow loop. This is equivalent to trapping some small fraction of the total number of particles on each pass and correspondingly decreasing the flow-through time. If we denote this decrement in the trapping fraction as $d$, then the trapping rate in the fully mixed case is give by

$$
\begin{aligned}
N_{p}(t) & =\lim _{d \rightarrow 0} N_{p}(0)\left(1-d f_{t}\right)^{\frac{t}{d t_{i}}} \\
& =N_{p}(0)\left(\lim _{d \rightarrow 0}\left(1-d f_{t}\right)^{\frac{1}{-d f t_{i}}}\right)-\frac{t f_{t}}{t_{i}} \\
& =\mathrm{N}_{\mathrm{p}}(0) \mathrm{e}^{-\mathrm{f}_{\mathrm{t}} \mathrm{t} / \mathrm{t}_{\mathrm{i}}} .
\end{aligned}
$$

Equations 15 and 16 give bracketing expressions for the number of particles in suspension vs. time. In the non-mixed case, the number of particles in suspension is diminished by $\left(1-f_{t}\right)$ on each cycle, while in the fully mixed case the number is diminished by $e^{-f t}$ on each pass.

Table 13 gives the fraction of particles passing through the system per flow-through time, $t_{i}$, per minute under these assumptions for each of the flow rates. It is interesting to note that, in this particular experimental setup, which consists of a closed loop flow rig with a recirculating particle slurry, and under the stated modeling assumptions, the flow rate does not have a dramatic affect on the rate at which particles are collected. This is seen Table 14, which shows the fraction of particles that would remain in suspension after $4 \mathrm{~min}$ for each of the cases considered. We see that, although the fraction captured per flow-through time decreases with increasing flow rate (cf., Table 11), the total capture rate remains roughly unchanged, since the higher flow rates correspond to a greater number of passages through the system per unit time. In essence, the particle capture rate is determined by residence time (and mixing conditions) in a closed-loop system; this is independent of the speed at which particles pass over the magnet.

Table 13. Particle transmission rates.

\begin{tabular}{|c|c|c|c|c|}
\hline $\begin{array}{c}\text { Flow Rate } \\
(\mathrm{mL} / \mathrm{min})\end{array}$ & $\begin{array}{c}t_{i} \\
(\mathrm{~min})\end{array}$ & $f_{\mathrm{p}}$ & $\begin{array}{c}\text { Fully } \\
\text { Mixed }\end{array}$ & $\begin{array}{c}\text { No Axial } \\
\text { Mixing }\end{array}$ \\
\hline 50 & 4 & 0.498 & 0.605 & 0.502 \\
\hline 100 & 2 & 0.274 & 0.760 & 0.726 \\
\hline 200 & 1 & 0.152 & 0.859 & 0.848 \\
\hline
\end{tabular}


Table 14. Remaining particle fraction after 4 minutes.

\begin{tabular}{|c|c|c|}
\hline $\begin{array}{c}\text { Flow Rate, } \\
\mathrm{mL} / \mathrm{min}\end{array}$ & $\begin{array}{c}\text { Fully } \\
\text { Mixed }\end{array}$ & $\begin{array}{c}\text { No Axial } \\
\text { Mixing }\end{array}$ \\
\hline 50 & 0.605 & 0.502 \\
\hline 100 & 0.578 & 0.527 \\
\hline 200 & 0.554 & 0.517 \\
\hline
\end{tabular}

The independence of transmission fraction in Table 14 is at odds with experimental evidence, which suggests that no particle trapping occurs at sufficiently high flow speeds. Possible explanations for this are:

- The trend in Table 14 does not continue at higher flow speeds. (Initial tests reveal that the trend does continue.)

- At higher speeds, the flow becomes turbulent, with increased mixing and a qualitative change in particle settling behavior.

- Particles subjected to wall shear stress on the glass sidewalls of the test section slide and are not immediately trapped, as assumed in the preceding computations.

Of these, the first two items are probably not of clinical relevance, although it is possible to have turbulence in the presence of a stenosis or surgical intervention such as a graft or stent, and this should be considered in any patient-specific modeling. To adequately address the third item, we would need an improved particle-wall model, e.g., based on lubrication theory. (This is currently an active research area.) However, once the particle pile is initiated, or in the presence of a compliant vascular wall, such a model is probably unnecessary and the simple sticking condition should suffice. 


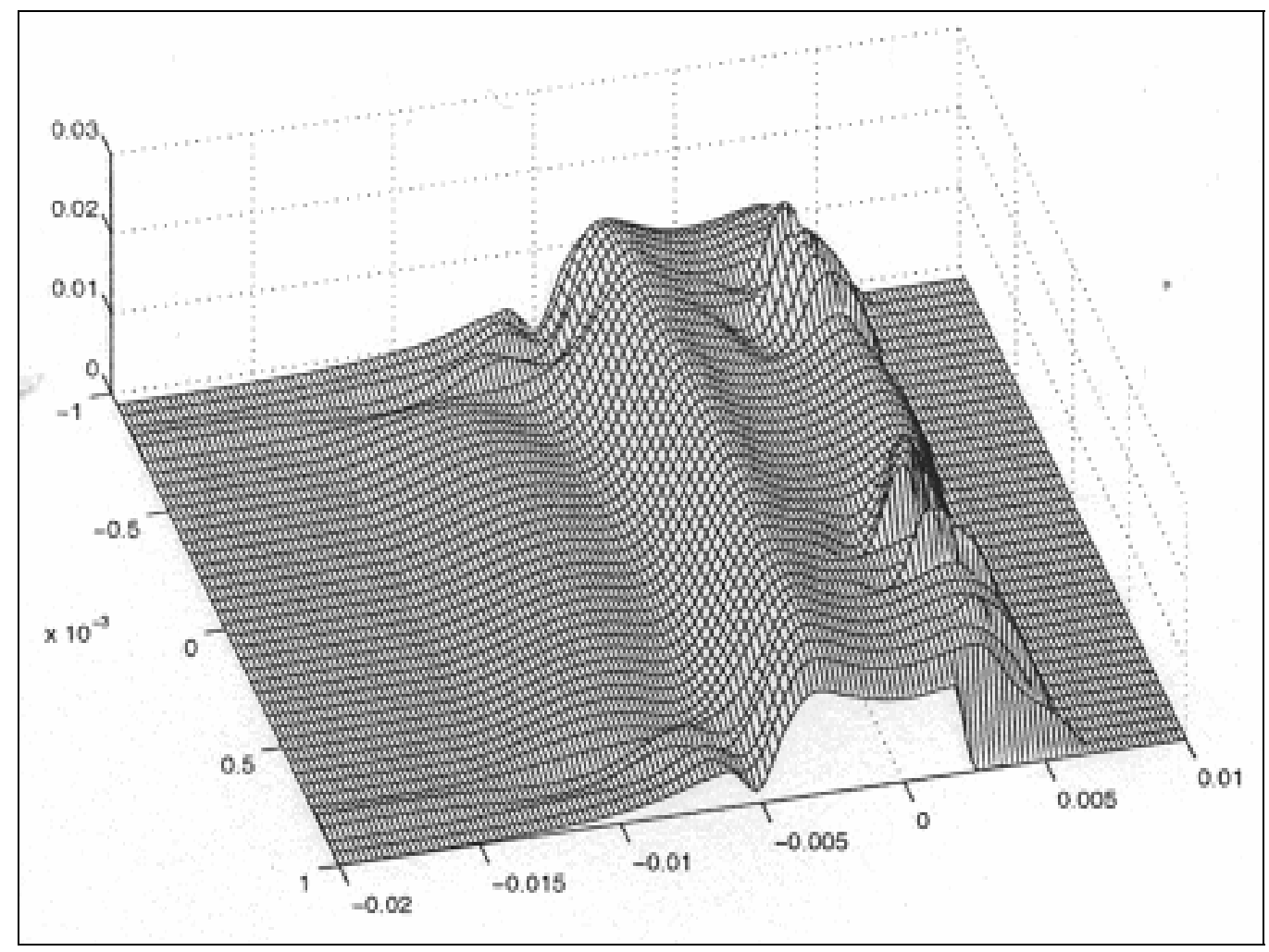

Figure 29. Predicted deposition pattern for $100-\mathrm{mL} / \mathrm{min}$ case.

Note difference in $x$ and y scales (in meters).

\section{e. Particle Deposition}

We have computed the expected particle deposition pattern as follows. In the cross-sectional plane at $x=-30 \mathrm{~mm}$, we release a particles $\rho_{j k}$ each $\left(y_{j}, z_{k}\right)$ location in the $m \times m$ inlet grid. The incoming particle flux, in particles per second, is $\rho_{p} w_{j k} \mathrm{~A}_{\text {cell }}$, where $\mathrm{A}_{\text {cell }}=(0.002 \mathrm{~m})^{2} / \mathrm{m}^{2}$. Focusing on one column, $y_{i}$ fixed, we find that the captured particles are spread out in the axial direction, with final position given by $(x, y, z)=\left(x_{j k}, y_{j}, 0.001\right)$, with $x_{j k}$ a computed function of the input point. We compute the midpoint, $x_{j+1 / 2}$, and distance $d_{j+1 / 2}$ between adjacent particles trapped on the upper channel surface. The flux onto the patch $\left[x_{i, j-1 / 2}, x_{i . j+1 / 2}\right] \times\left[y_{j-1 / 2}, y_{j+1 / 2}\right]$ is $\rho_{p} w_{j k} \mathrm{~A}_{\text {cell }}$. Hence, the local deposition rate at $\left(x_{j k}, y_{j}, 0.001\right)$, in particles $/ \mathrm{sec}-\mathrm{m}^{2}$, is

$$
\frac{\rho_{p} w_{j k} \mathrm{~A}_{\text {cell }}}{\left(x_{i j+1 / 2}-\mathrm{x}_{\mathrm{ij}-1 / 2}\right) \times\left(y_{j+1 / 2}-y i_{-1 / 2}\right)} .
$$

Figure 21 shows the predicted deposition pattern for the $100-\mathrm{mL} / \mathrm{min}$ case. The predicted accumulation rates seem to be higher than what has been observed in the experiments. For example, Table 15 indicates that roughly $40-50 \%$ of the particles should be captured within the first 4 min of bringing the magnet in position in the flow rig.

The way in which the experiment is started might have an influence on the accumulation rate. For example, if the magnet is first positioned above the test rig with no flow, and the particles are concentrated in the mixing volume (jar), then there may be a large bolus of particles that pass by the magnet during the first flow cycle, resulting in a very high value of $\rho_{p}(t)$ at early times. This 
scenario, however, would indicate higher particle capture rates than predicted, not lower, so it seems unlikely that this is a source of discrepancy.

\section{CLINICAL TRIALS}

\section{a. Introduction}

The ability to utilize external magnetic fields to non-invasively guide, concentrate, and actively deliver medicated magnetic particles after simple intravenous injection to any location within the human body and independent of conventional physiological and pharmacological barriers is of great clinical importance to almost all medical specialties. Such a delivery method would confer a magnitude of advantages that directly translate to improved diagnostic and therapeutic options benefiting a wide variety of patients. Clearly, the clinically most important progress that magnetically guided, targeted drug therapy would offer are those described below:

Non-invasiveness: This drug delivery method differs fundamentally from current delivery systems, which are either based on non-specific targeting supported only by random drug distribution throughout the body, carrier- or receptor-mediated drug concentration at the target tissue site, or invasive methods such as intravascular, catheter-based delivery. Each current method has its own inherent and specific application limitations (the outline of those is beyond the scope of this text); generally, the methods are either passive (that is, dependent on physiological or pathophysiological processes and hence more difficult to control) or invasive (i.e., placement of a catheter, exposing the patients to additional risks). In contrast, magnetically guided drug delivery would be the first delivery method (excluding radiation therapies) that combines two important clinical advantages: active and non-invasive drug delivery.

Target selectivity: Externally applied magnetic fields can be designed in geometry and strength in a wide variety of arrangements accommodating organ-specific anatomical and physiological drug delivery needs. This facilitates selective magnetic particle (and hence, drug) concentration at and transport into well-defined target tissue areas of interest. Additionally, future bioengineering development will allow combining conventional technologies with magnetic drug delivery to increase target selectivity and precision. Such examples include magnetic resonance imaging (MRI) to more precisely configure magnetic field properties needed for an individual drug delivery, and MRI real-time monitoring of drug accumulation within target tissues, as well as activation drug release at the target site using focused radiofrequency-generated thermal energy.

Drug barriers: As magnetic fields do not have physical barriers within the human body, conventional obstacles (i.e., skull bone for x-rays) that possibly could inhibit external magnetic guidance are basically non-existing, further promoting the uniqueness of the proposed method. In addition, magnetic gradients can be utilized for active drug delivery though internal barriers such as the inner lining of the vascular system. For example, the brain is uniquely sheltered from almost all foreign and physiologically blood-circulating substances by a tight cell barrier surrounding all capillaries and other brain vessels, the blood brain barrier (BBB). Many drugs either will not at all or only in insufficient concentrations reach the brain tissue due to the BBB's selective permeability. For instance, nerve growth factor (NGF) promotes cell and synaptic brain recovery after injuries but, unfortunately, is excluded by the BBB and currently no suitable 
method exists to deliver NGF across the BBB and to promote brain recovery after injury. However, magnetic guidance of NGF-medicated magnetic particles could theoretically be useful for NGF delivery across the barrier and into the brain tissue. Such investigations are currently underway in our laboratories.

Non- or poorly perfused tissues provide additional "barriers" to effective drug treatment, as diminished or lack of blood perfusion will decrease the drug concentration at the target tissue. For example, the "clot buster" tissue plasminogen activator (tPA) has selectivity for fresh thrombi within the vasculature and is commonly used to treat acute heart and brain arterial occlusions. However, the clinical success of effective tPA clot lysis is reduced by insufficient tPA delivery into the clot because of stagnant blood flow. One can envision that magnetically guided tPA delivery would not only be able to focally concentrate the clot buster at the vascular occlusion site but also promote active delivery into dead clot tissue despite the lack of blood flow, hence increasing vascular lysis rate.

Figure 30 summarize some of the advantages magnetically guided drug delivery offers in comparison with conventional therapy, using as an example clot lysis of a brain artery occlusion in an acute stroke victim.

\section{b. Large-Animal Studies}

We proposed that an externally applied magnetic force (that is, a designer magnet or array of magnets strategically positioned outside of the body and close to the skin surface) would be able to focally concentrate freely blood-circulating magnetic particles even in large animals, such as non-human primates.

To investigate the general feasibility to concentrate non-medicated magnetic particles within the blood stream, we performed a series of simple experiments in the large animal model: monkeys ( 3 to $5 \mathrm{~kg}$ body weight) were injected over 10 minutes intra-arterially (abdominal aorta; $\mathrm{n}=2$ ) and intravenously (subclavian vein; $\mathrm{n}=2$ ) with $10 \mathrm{mg}$ of cellulose-based PEG-ylated magnetic particles. Prior to injection a 0.45 Tesla permanent magnet was externally fixated on the skin at the inner thigh region exactly above the femoral artery. Ultrasound was used to identify exact magnet placement and to measure the femoral artery blood flow peak velocities, which ranged from 90 to $110 \mathrm{~cm} / \mathrm{sec}$. After a 10 minutes circulation time the animal was sacrificed. We performed histopathological examination of sections from the proximal legs at the level of magnet placement and from the same area of the contralateral leg (control, no magnet used).

Histology [Hematoxylin-Eosin (H\&E) stain] verified intra-arterial particle concentration (identified as black particle agglomerates on staining) at the arterial region above the surface magnet (Figure 31a); particles were neither identified at arterial segments proximal or distal to the magnet nor in the contralateral control leg (Figure 31b). In addition, arterial concentration was also shown in small brain arteries after positioning a second 0.45 -Tesla magnet directly on the brain surface in the two animals receiving venous particle injection (Figure 31c). These simple experiments identify successful sequestration of magnetic particles in larger and smaller arterial branches after arterial upstream and systemic venous injection. Future magnetic guidance systems will likely employ adjustable three-dimensional magnetic fields to direct, sequestrate, and trigger drug release of medicated magnetic particles. Importantly, these 
experiments also demonstrated the need to carefully select particles with a well-defined size distribution, as two animals experienced acute respiratory distress after particle injection because of widespread pulmonary capillary occlusions due to intravascular particles (Figure 31d). Electron microscopic verification (Figure 31e) of the particles marketed as " $3 \mu \mathrm{m}$ " particles identified a broad size distribution ranging from 1 to $20 \mu \mathrm{m}$, making such particles of little use for future experiments.

\section{Acute Ischemic Stroke}

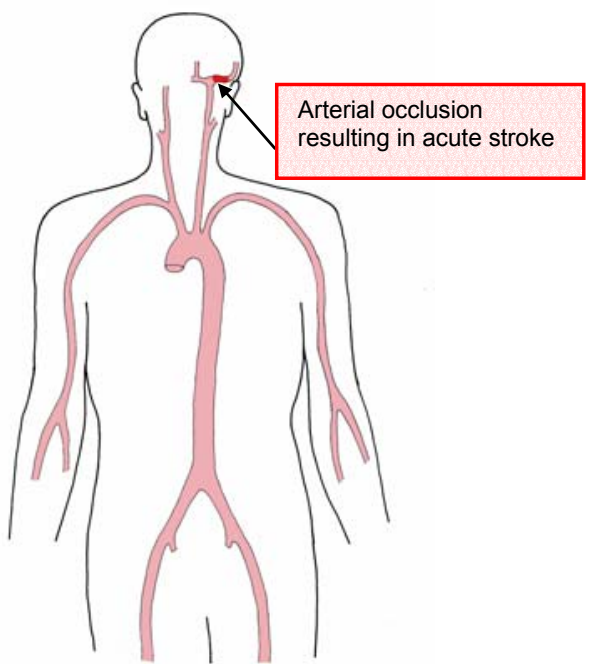

Rendition of a typical stroke scenario. The occlusion of a major brain artery will lead to a devastating neurological deficit unless emergently treated with "clot-busting" drugs such as tissue plasminogen activator (t-PA), offering an approximately 1 out of 3 chance to reopen the artery and reperfuse the affected brain tissue. Plasminogen activators provide the only therapeutic option to treat the primary lesion, the vessel occlusion.

Systemic Thrombolysis

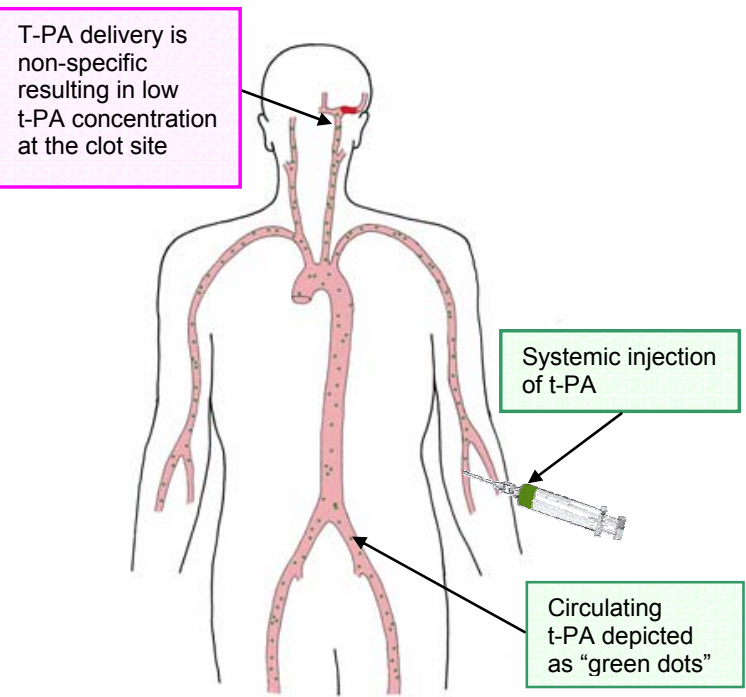

If the acute stroke patient arrives within 3 hours after onset, t-PA can be injected intravenously. Systemic injection is non-invasive, a major advantage. However, as this therapy is not targeted directly at the clot, relatively high systemic t-PA dosages are required, increasing bleeding complications and decreasing overall clot lysis efficiency. Unfortunately, most patients do not arrive in time to be considered for this kind of therapy.

Figure 30. Comparison of current and proposed therapies for treating acute stroke. 


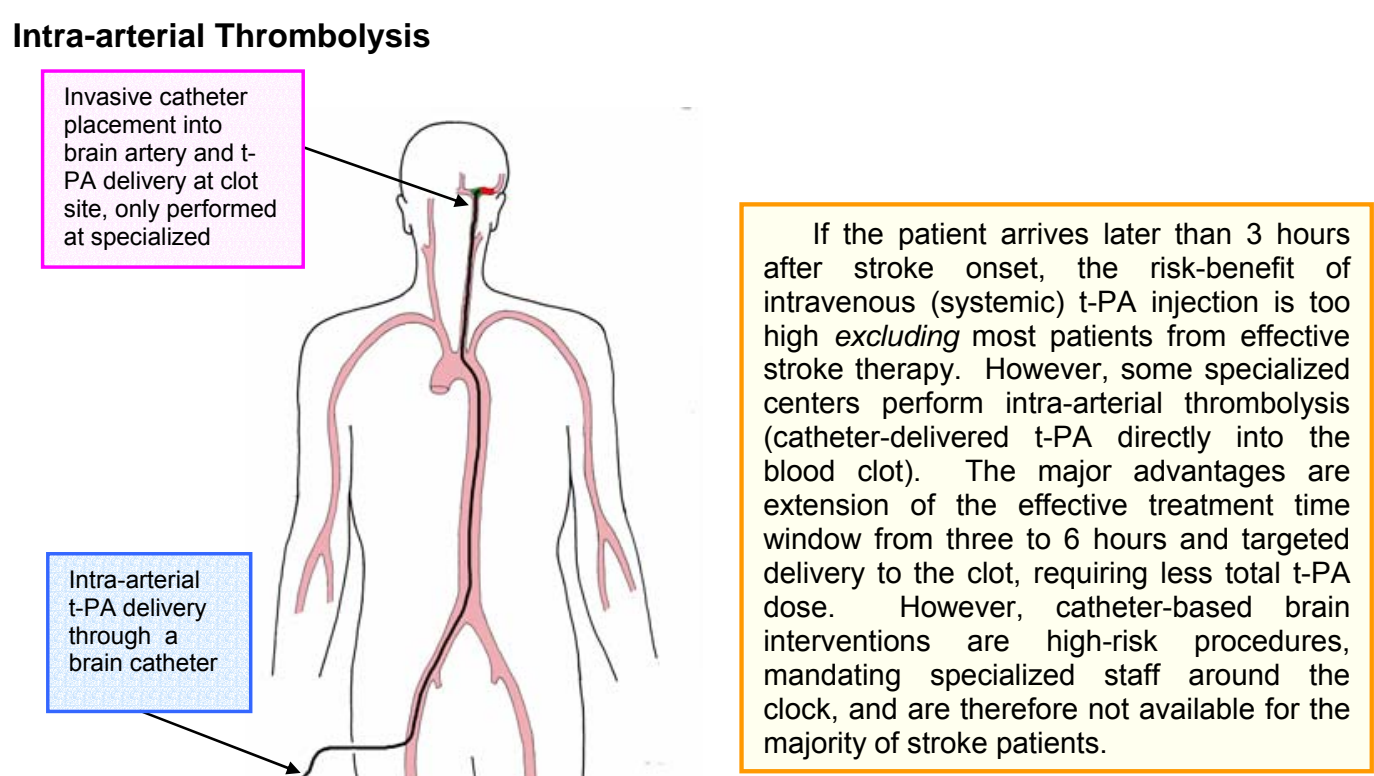

Proposed System: Magnetically Guided Thrombolysis

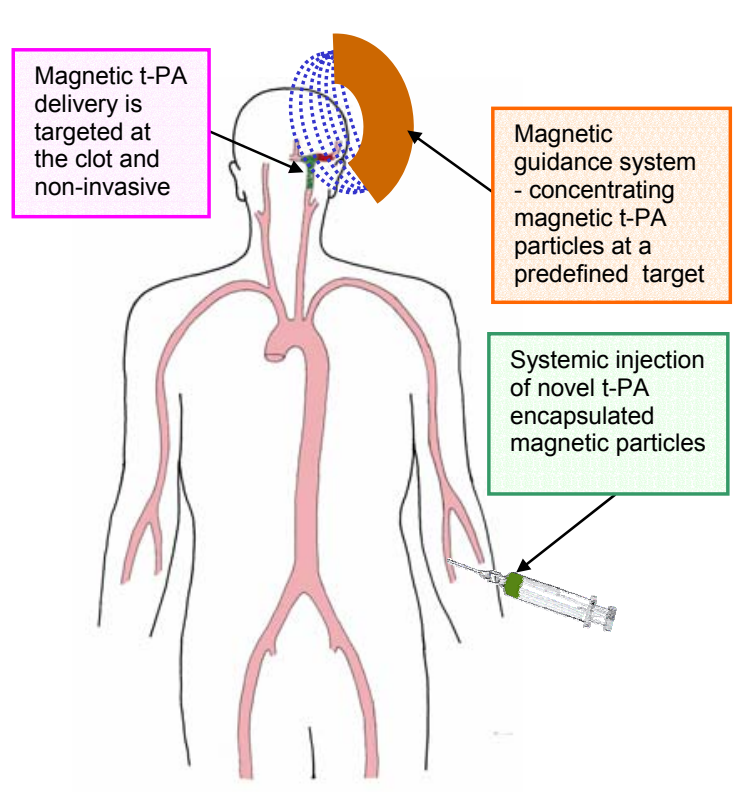

The proposed drug delivery system will minimize therapy risk and extend the therapeutic window by combining the advantages of intravenous and catheterbased t-PA delivery. A small dose of designer magnetic particles containing encapsulated, active t-PA is injected systemically into the vein (non-invasiveness) and magnetically guided and trapped at the site of the blood clot by externally applied magnetic fields. Plasminogen activator release from the particle matrix is spontaneous or ultrasound enhanced. As tPA encapsulated particles are magnetically guided to and concentrated at the clot site, successful lysis can be achieved with a relatively small dose of intravenously injected t-PA. This delivery concept may lead to decreased systemic side effects and increasing time window for successful treatment.

Figure 30. Comparison of current and proposed therapies for treating acute stroke (continued). 

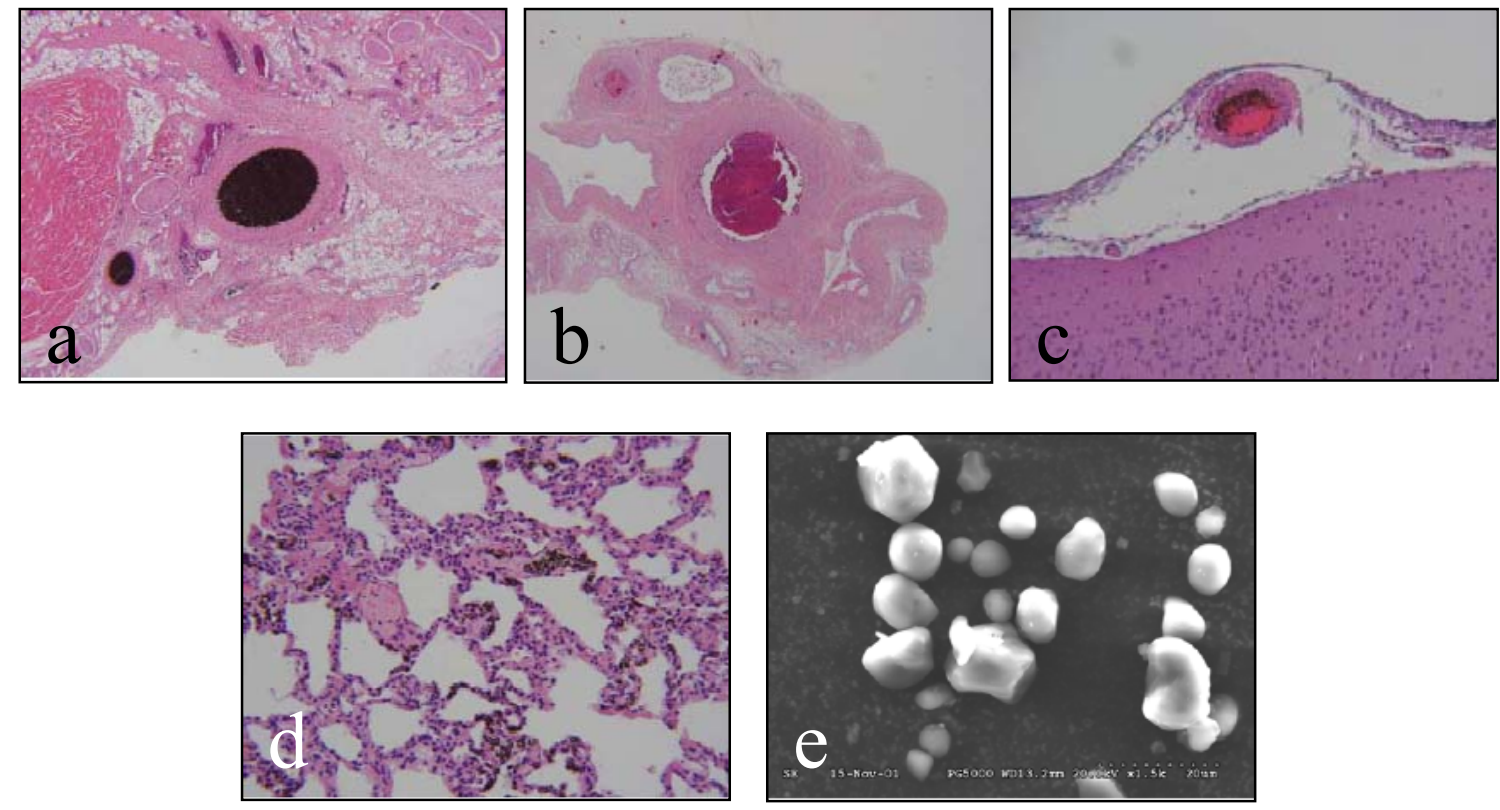

Figure 31. Histology-verified intra-arterial particle concentration (identified as black particle agglomerates on H\&E staining) at arterial region above surface magnet.

\section{c. Rodent Studies}

Additionally, we investigated pharmacokinetic properties of various magnetic particles in the rat animal model. However, these data are an essential component of our research investigating nanotechnology-based detoxification and therefore will be extensively discussed in a separate publication. 


\section{SUMMARY AND FUTURE DIRECTIONS}

We have made good progress in developing this program and transitioning Department of Energy-funded basic and engineering technologies to research in the medical sector. We have established partnerships with universities, medical institutions, and industry to increase our chance for success. The summary review provided here serves as a basis for continued progress in areas of nanoparticle synthesis and drug encapsulation, magnetic nanoparticle options and developments, magnetic modeling, and in vitro flow dynamics. It will also serve as a quick reference in particle synthesis as new experiments are needed, to troubleshoot experimental problems, and to propose new drug delivery concepts.

Our progress can be summarized as follows:

- We have established unique laboratory capabilities at Argonne and with our research partners that provide an array of synthesis, analytical, modeling, and engineering tools needed for success in this multi-disciplinary endeavor.

- We have made some progress in defining clinically relevant magnet configurations that will maximize particle accumulation in the region of interest. We are building a laboratory devoted to in vitro and in vivo testing of magnet arrays at The University of Chicago that will provide valuable data concerning the expectations and limitations of magnetic targeted delivery.

- We are beginning to understand mechanistically how magnetic particles will behave during vascular transport and under the influence of externally-applied magnetic field gradients. This capability will eventually lead to predictive models that define clinical protocol.

- Our in vitro testing facility provides remarkable versatility in testing prototype magnetic particles prior to animal testing and serves as a benchmark system for validating the predictive computer models. Ongoing tests will expand the experimental variables to include rounded cells, bifurcations, viscous fluids, and variable magnetic fields and gradients.

- Highly uniform, highly magnetic nanoparticles are being developed to enhance the magnetic moment of the polymeric-based micro/nanoparticles. Moreover, control of the size of the magnetic nanoparticles should increase the uniformity of the magnetic moments of individual particles and facilitate predictive modeling.

- We are beginning to understand the parameters involved in creating high-yield, uniform, magnetic micro/nanoparticles capable of efficient encapsulation of hydrophilic drugs or radionuclides. We recently began synthesizing custom biodegradable, co-block polymers to meet the complicated needs of various clinical scenarios (e.g., size uniformity, deliver hydrophilic drug, degrade uniformly over time).

- Our clinical trials support our research objectives and provide initial proof that magnetic particles are capable of targeting specific tissues and vascular regions. Our access to various animal models is invaluable to our continued progress. 


\section{ACKNOWLEDGEMENTS}

The authors extend a special thanks to Drs. H. Drucker, G. Crabtree, and R. Poeppel for their support and advice. The authors also thank H. Claus and Dexter Magnetics, Inc. for magnetic field measurements. The authors acknowledge V. Strezo and S. Zussman for their help in preparing this document.

Research supported by the U.S. Department of Energy under Contract W-31-109-Eng-38. 


\section{REFERENCES}

Alexiou, C., W. Arnold, R. J. Klein, F. G. Parak, P. Hulin, C. Bergemann, W. Erhardt, S. Wagenpfeil, and A. S. Lübbe. 2000. "Locoregional Cancer Treatment with Magnetic Drug Targeting," Cancer Research, 60, 6641.

Alexiou, C., W. Arnold, P. Hulin, R. J. Klein, H. Renz, F. G. Parak, C. Bergemann, and A. S. Lü bbe. 2001. 2001. "Magnetic Mitoxantrone Nanoparticle Detection by Histology X-Ray and MRI after Magnetic Tumor Targeting," JMMM, 225, 187.

Allemann, E., R. Gurny, and E. Doelkerr. 1993. "Drug Loaded Nanoparticles: Preparation Methods and Drug Targeting Issues," Eur. J. Pharm. Biopharm, 39, 173.

Arias, J. L., V. Gallardo, S. A. Gomez-Lopera, R. C. Plaza, and A. V. Delgado. 2001. "Synthesis and Characterization of Poly(ethyl-2-cyanoacrylate) Nanoparticles with a Magnetic Core," J. Controlled Release, 77, 309-321.

Arshady, R. 1990. "Microspheres and Microcapsules: a Survey of Manufacturing Techniques, Part 3. Solvent Evaporation,” Polym. Eng. Sci., 30, 915.

Arshady, R. 1993. "Microspheres for Biomedical Applications: Preparation of Reactive and Labeled Microspheres," Biomaterials, 14, 5.

Arshady, R., and M. Monshipouri. 1999. Targeted Delivery of Microparticulate Carriers. In: R. Arshady Microspheres Microcapsules and Liposomes, (vol. 2). pp. 403-432, London, UK: Citus Books.

Babincová, M., P. Sourivong, D. Chorvát, and P. Babinec. 1999. "Laser Triggered Drug Release from Magnetoliposomes," JMMM, 194, 163.

Bai, G.R., L. J. Thompson. and J. A. Eastman. 2002. Argonne National Laboratory, unpublished data.

Bazile, D. V., C. Ropert, P. Huve, T. Verrechia, M. Marland, A. Frydman, M., A. Frydman, M. Veillard and G. Spenlehauer. 1992. Body Distribution of Fully Biodegradable [14C]Poly(lacticacid) Nanoparticles Coated with Albumin after Parenteral Administration to Rats, Biomaterials 13, 1093.

Beck, L. R., V. Z. Pope, D. R. Cowsar, D. H Lewis, T. R. Tice, [Title], Adv. Contracept. Deliv. Systems 1, 79.

Bhadra, B., S. Bhadra, P. Jain and N.K. Jain. 2002. "Pegnology: a Review of PEG-Ylated Systems," Parmazie 57, 5.

Bitz, C., and E. Doelker. 1996. "Influence of the Preparation Method on Residual Solvent in Biodegradable Microspheres,” Int. J. Pharm., 131, 171. 
Blanchard, R. J. W., I. Grotenhuis, J.W. LaFave, and J.F. Perry, Jr. 1975. Proc. Soc. Exp. Biol. Med. 118, 465.

Bleich, J., and B.W. Mueller. 1996. "Production of Drug Loaded Microparticles by the Use of Supercritical Gases with the Aerosol Solvent Extraction (ASSES) Process," J. Microencapsulation, 14, 131.

Bodmeier, R., and J.W. McGinity. 1988. "Solvent Selection in the Preparation of Poly(DLlactide) Microspheres Prepared by the Solvent Evaporation Technique," Int. J. Pharm., 43, 197.

Brindley, A., S.S. Davis, M.C. Davies, and J.F. Watts. 1995. "Polystyrene Colloids with Surface-Grafted Polyethylene Oxide as Model Systems for Site-Specific Drug Delivery,” Journal of Colloid and Interface Science, 171, 150.

Bulte, J. W. M., M. de Cuyper, D. Despres, and J.A. Frank. 1999. "Preparation, Relaxometry, and Biokinetics of PEGylated Magnetoliposomes as MR Contrast Agent," JMMM, 194, 204.

Chan, T. W., C. Eley, P. Liberti, A. So, and H.Y. Kressel. 1992. "Magnetic Resonance Imaging of Abscesses using Lipid-Coated Iron Oxide Particles,” Invest. Radio., 29, Suppl 2, S87.

Chang, W., G. Skandan, S. C. Danforth et al. 1995. Nanostructured Ceramics Synthesized by Chemical Vapor Condensation, NanoStructured Materials 6, 321.

Chen, Z.-P., R.B. Roemer, and T.C. Cetas. 1992. Med. Phys. 19, 989.

Choi, C.J., Dong X. L. and Kim B. K. 2001a. Microstructure and Magnetic Properties of Fe Nanoparticles Synthesized by Chemical Vapor Condensation, Materials Transactions 42, 2046.

Choi, C.J., Dong X. L. and Kim B. K. 2001b. Characterization of Fe and Co Nanoparticles Synthesized by Chemical Vapor Condensation, Scripta Materialia 44, 2225.

Cleland, J. L. 1995. "Design and Production of Single Immunization Vaccines Using Polylactide Polyglycolide Microspheres Systems,” Pharm. Biotechnol., 6, 439.

Couvreur, P, B. Kante, M. Roland, P. Guiot, P. Bandhuin, P. Speiser, "Polycyanoacrylate Nanocapsules as Potential Lysosomotropic Carriers: Preparation, Morphological and Sorptive Properties," J. Pharm. Pharmacol. 31331.

Cui, C., and S. P. Schwendeman. 2001. "Surface Entrapment of Polylysine in Biodegradable Poly(DL-lactide-co-glycolide) Microparticles," Macromolecules, 34, 8426.

Donini, C., D. N. Robinson, P. Colombo, F. Giordano, and N.A. Peppas. 2002. Preparation of Poly(methacrlic acid-g-polyethylene glycol) Nanospheres from Methaxcrylic Monomers for Pharmaceutical Applications," Intern. J. Parmaceutics, 245, 83. 
Dreborg, S., and E.B. Akerblom. 1990. "Immunotherapy with Monomethoxy Polyethylene Glycol Modified Allergens,” Crit. Rev. Ther. Drug Carrier Syst., 6, 315.

Duncan, R., and J. Kopecek, "Soluble Synthetic Polymers as Potential Drug Carriers," Adv. Polym. Sci., 57, 53.

Dunn, S. E., A. G. A. Coombes, M. C. Garnett, S. S. Davis, M. C. Davies, and L. Illum. 1997. "In Vitro Cell Interactions and Biodistribution of Poly(Lactide-co-Glycolide) Nanospheres Surface Modified by Poloxamer and Poloxamine Copolymer," J. Controlled Release, 44, 65.

Efimov, V. A., I.N. Pashkova, A.L. Kalinkina, and O.G. Chakhmakhcheva. 1993. "Synthesis of Conjugates of Oligonucleotides with Polyethylene Glycol," Bioorgan Khimiya, 19, 800.

Ehrhardt, G. J., and D.E. Day. 1987. "Therapeutic use of ${ }^{90} \mathrm{Y}$ Microsperes," J. Med Biol., 14, 233.

Erbas, B., et al., 2001. "Biodistribution and Localization of Radiolabeled Microparticles," Citus Books, London, MMM Series, 3, 249.

Eyles, J. E., H.O. Alpar, B.R. Conway, and M. Keswick. 1997. "Oral Delivery and Fate of Poly(lactic acid) Microspheres-Encapsulated Interferon in Rats,” J. Pharm. Pharmacol, 49, 669.

Fessi, H., Puisieux, J.Ph. Devissaguet, N. Ammoury, S. Benita, "Nanocapsule Formation by Interfacial Polymer Deposition Following Solvent Displacement,” Int. J. Pharm., 55, R1.

Feakes, D. A., K. Shelly, C.B. Knobler, and M. F. Hawthorne. 1994. " $\mathrm{Na}_{3}\left[\mathrm{~B}_{20} \mathrm{H}_{17} \mathrm{NH}_{3}\right]$ : Synthesis and Liposomal Delivery to Murine Tumors," Proc. Nat. Acad. Sci., 91, 3029.

Fu, K., K. Griebenow, L. Hsieh, A. M. Klibanov, and R. Langer. 1999. FTIR Characterization of the Secondary Structure of Proteins Encapsulated within PLGA Microspheres, J. Controlled Release, 58, 357.

Gaur U., S. K. Sahoo, T. K. De, P. C. Ghosh, A. Maitra, and P. K. Ghosh. 2000. Biodistribution of fluoresceinated dextran using novel nanoparticles evading reticuloendothelial system. 2000. Int. J. Pharm., 202 (2000) 1-10.

Gander, B., E. Wehrli, R. Alder, and H. P. Merkle. 1995. "Quality Improvement of Spray Dried Protein Loaded D,L-PLA Microspheres by Appropriate Polymer Solvent Selection," J. Microencapsulation, 12, 83.

Gillies, G. T., R. C. Ritter, W. C. Broaddus, M. S. Grady, M. A. Howard III, and R. G. McNeil. 1994. Magnetic manipulation instrumentation for medical physics research, Review of Scientific Instruments 65 (1994), 533-62.

Goldenberg, D. M., and G. L Griffiths. 1992. "Radioimmunotherapy of Cancer: Arming the Missiles," J. Nucl. Med. 33, 1110. 
Goldfrank, L. 1998. "Part D. Clinical Basis for Medical Toxicology,” in Goldfrank's Toxicologic Emergencies, $6^{\text {th }}$ Edition, Appleton \& Lange, 620.

Gomez-Lopera, S. A., R. C. Plaza and, A. V. Delgado. 2001. "Synthesis and Characterization of Spherical Magnetite/Biodegradable Polymer Composite Particles," J. Colloid. Interf. Sci., 240, 40.

Goodwin, S., C. Peterson, C. Hoh, and C. Bittner. 1999. "Targeting and Retention of Magnetic Target Carriers (mtcs) Enhancing Intra-Arterial Chemotherapy,” JMMM, 194, 132.

Gref, R., Y. Minamitake, M.T. Peracchia, V. Trubetskoy, V. Torchilin, and R. Langer. 1994. "Biodegradable Long-Circulating Polymeric Nanospheres," Science, 263, 1600.

Gref, R., A. Domb, P. Quellec, T. Blunk, R. H. Miller, J. M. Verbavatz, and R. Langer. 1995. "The Controlled Intravenous Delivery of Drugs Using PEG-Coated Sterically Stabilized Nanospheres," Advanced Drug Delivery Reviews 16, 215.

Grüttner, C., J. Teller, W. Schütt et al. 1997. in: U. Häfeli, W. Schütt, J. Teller, M. Zborowski (eds.), Scientific and Clinical Applications of Magnetic Carriers, Plenum Press, New York, pp. 53.

Grüttner, C., and J. Teller. 1999. "New Types of Silica-Fortified Magnetic Nanoparticles as Tools for Molecular Biology Applications," JMMM, 194, 8.

Gupta, P. K., and C. T. Hung. 1990. "Targeted Delivery of Low Dose Doxorubicin Hydrochloride Administrated Via Albumin Microspheres in Rats," Journal of Microencapsulation, 7, 85 .

Gupta, P. K., and C.T. Hung. 1994. Magnetic Controlled Targeted Chemotherapy,. In: N. Willmott and J. Daly (eds.), Microspheres and Regional Cancer Therapy, CRC Press, Inc., Boca Raton, FL, pp. 1-59.

Häfeli, U. O., S. M. Sweeney, B. S. Beresford, E. H. Sim, and R. M Macklis. 1994. "Magnetically Directed Poly(lactic acid) ${ }^{90}$ y-Microcapsules: Novel Agents for Targeted Intracavitary Radiotherapy,” Journal of Biomedical Materials Research, 28, 901.

Häfeli, U. O., G.J. Pauer, and R.M. Macklis. 1995. "Treatment of Mouse Tumors with Radioactive Magnetic Microcapsules: Model for Intracavitary Radiotherapy,” Proc. Internat. Symp. Control. Rel. Bioact. Mater., 22, 89.

Häfeli, U. O., and G.J. Pauer. 1999. "In Vitro and Toxicity of Magnetic Microspheres," JMMM, $194,76$.

Häfeli, U., G. Pauer, S. Failing, and G. Tapolsky. 2001. "Radiolabeling of Magnetic Particles with Rhenium-188 for cancer therapy,” JMMM, 225, 73. 
Häfeli, U.O. 2003. Cleveland Clinic Hospital, unpublished data.

Harris, J. 1985. "Laboratory Synthesis of Polyethylene Glycol Derivatives," J. Macromol. Sci.Rev. Macromol. Chem. Phys. C25, 325.

Hasirci, V., K. Lewandrowski, J. D. Gresser, D. L. Wise, and D.J. Trantolo. 2001. "Versatility of Biodegradable Biopolymers: Degradability and an In Vitro Application," Journal of biotechnology 86, 135 .

Hoste, K., D. Bruneel, A. De Marre, F. De Schrijver and E. Schacht. 1994. "Synthesis and Characterization of Poly(oxyethylene) Modified Dextrans," Macromol. Rapid Commun., 15, 697.

Huang, S. K., E. Mayhew, S. Gilani, D. D. Lasic, F. J Martin, and D. Papahadjopoulos. 1992. "Parmacokinetics and Therapeutics of Sterically Stabilized Liposomes in Mice Bearing C-26 Colon Carcinoima," Cancer Research, 52, 6774.

Hurwitz, E., R. Levy, R. Maron, M. Wilchek, R. Arnon, and M. Sela. 1975. Cancer Research, 35, 1175 .

Iannone, A., R.L. Magin, T. Walczak, M. Federico, H.M. Swartz, A. Tomasi, and V. Vannini. 1991. "Blood Clearance of Dextran Magnetite Particles Determined by a Non-Invasive ESR Method," Mag. Reson. Med., 22, 435.

Illum, L., I.M. Honneyball, and S.S. Davis, "The Effect of Hydrophilic Coatings on the Uptake of Colloidal Particles by the Liver and by Peritoneal Macrophages," Int. J. Pharm., 29, 53.

Illum, L., S. S. Davis, R. H. Muller, E. Mark, and P. West. 1987. "The Organ Distribution and Circulation Time of Intravenously Injected Colloidal Carriers Sterically Stabilizer with a Bloc Copolymer-Poloxaamine 908," Life Science, 40, 367.

Jeon, S. I., and J.D. Andrade. 1991. "Protein-Surface Interactions in the Presence of Polyethylene Oxide. I \& II. Effect of Protein Size,” J. Colloid Interf. Sci. 142, 149-166.

Jordan, A., P. Wust, R. Scholz et al. 1997. in: U. Häfeli, W. Schütt, J. Teller, M. Zborowski (eds.), Scientific and Clinical Applications of Magnetic Carriers, Plenum Press, New York, pp. 569.

Jung, C. W., and P. Jacobs. 1995. "Physical and Chemical Properties of Superparamagnetic Iron Oxide MR Contrast Agents: Ferumoxides, Ferumoxtran, Ferumoxsil," Magnetic Resonance Imaging 13, 661.

Kaminski, M. D. 2003. Argonne National Laboratory, unpublished data. 
Kane, B. 1992. "Drug Therapy for Colorectal Cancer," from coverage of the 38th Annual Meeting of the American Society of Clinical Oncology, 38th Annual Meeting of the American Society of Clinical Oncology, in Medscape.

Kronick, P. L., G. L. Campbell, and K. Joseph. 1978. "Magnetic Microspheres Prepared by Redox Polymerization Used in a Cell Separation Based on Gangliosides," Science, 200, 1074.

Krause, H-J, A. Schwarz, and P. Rohdewald. 1985. "Polylactic Acid Nanoparticles, a Colloidal Drug Delivery System for Lipophilic Drugs,” Int. J. Pharm. 27, 145.

Kumar, M., N. V. R. 2000. "Nano and Microparticles as Controlled Drug Delivery Devices," J. Pharm. Pharm. Sci. 3(2):234-258.

Kuznetsov, A. A., V. I. Filippov, R. N. Alyautdin, N. L. Torshina, and O. A. Kuznetsov. 2001. "Application of Magnetic Liposomes for Magnetically Guided Transport of Muscle Relaxants and Anti-Cancer Photodynamic Drugs," Journal of Magnetism and Magnetic Materials, 225, 95.

Larson, S. 1991. "Radioimmunology - Imaging and Therapy," Cancer 67, 1253.

Lefort, J. 1869. "Memoire sur l'Oxyde de fer Magnetique et ses Combinaisons salines," Comptes Rendus Acad. Sci. Paris, 69, 179.

Li, X., Y. Zhang, R. Yan, W. Jia, Y. Minglong, X. Deng, and Z Huang. 2000. "Influence of Process Parameters on the Protein Stability Encapsulated in Poly-DL-Lactide-Poly(ethylene glycol) Microspheres," J. Controlled Release, 68, 41.

Lin, S.-Y., K.-S. Chen, H.-H. Teng, and M.-J. Li. 2000. "In vitro Degradation and Dissolution Behaviors of Microspheres Prepared by Three Low Molecular Weight Polyesters," J. Microencapsulation, 17 (5), 577.

Liu, Y., and X. Deng. 2002. "Influences of Preparation Conditions on Particle Size and DNALoading Efficiency for Poly(DL-Lactic Acid-Polyethyelen Glycol) Microspheres Entrapping Free DNA,” J. Controll. Release, 83, 147.

Lübbe, A. S., C. Bergemann, H. Riess, et al. 1996a. "Preclinical Experiences with Magnetic Drug Targeting: a Phase i Study with 4'-Epidoxorubicin in 14 Patients with Advanced Solid Tumors," Cancer Research, 56, 4686.

Lübbe, A. S., C. Bergemann, W. Huhnt, T. Fricke, H. Riess, J..W. Brock, and D. Huhn. 1996 b. "Preclinical Experiences with Magnetic Drug Targeting: Tolerance and Efficacy," Cancer Research, 56, 4694.

Mason, N. S., D.V.S. Gupta, D. W. Keller, R. S. Younquist, and R.E. Sparks. 1984. Microencapsulation of Progesterone for Contraception by Intracervical Injection, In: F. Lim (ed), Biomedical Applications of Microencapsulation, CRC Press, Boca Raton, FL 1984, 75-84. 
Massart, R. 1981. "Preparation of Aqueous Magnetic Liquids in Alkaline and Acidic Media," IEEE Trans. Magnetics 17(2), 1247.

Mausner, L. F., and S. C. Srivastava. 1993. "Selection of Radionuclides for Radioimmunotherapy," Am. Assoc. phys Med, 20, 503.

McGee, J. P., S. S. Davis, and D. T. Hagan. 1995. "Zero Order Release of Protein from Poly(D,L-lactide-co-glycolide) Microparticles Prepared Using a Modified Phase Separation Technique," J. Control Release, 34, 77.

Mendelsohn, J. 2002. "Targeted Therapies and Solid Tumors," Robert S. Mocharnuk MD, The Bernard L. Schwartz lecture given by Dr. John Mendelsohn, President of the University of Texas MD.

Mikhaylyk, O., A. Cherchenko, A. Ilkin, N. Dudchenko, V. Ruditsa, M. Novoseletz, and Y. Zozulya. 2001. "Glial Brain Tumor Targeting of Magnetite Nanoparticles in Rats," JMMM, $225,241$.

Miller, K., D. A. Vaena, and B. P. Schneider. 2002. "Target and Arrows; the Future of Breast Cancer Therapy," Medscape Hematology-Oncology e-Journal 5, 2.

Mochamuk, R. S. 2002. "Translational Research and Targeted Therapies," $2^{\text {nd }}$ Intern Lung Cancer Congress, in Medscape.

Moghimi, S. M., C. J. H. Porter, I. S. Muir, L. Illum and S. S. Davis. 1991. "Non-Phagocytic Uptake of Intravenously Injected Microspheres in Rat Sleen: Influence of Particle Size and Hydrophilic Coating," Biochemical and Biophysical Research Communications, 177 (2), 861.

Morita, T., Y. Horikiri, H. Yamahara, T. Suzuki, and H. Yoshimno. 2000a, "Formation and Isolation as Spherical Fine Protein Microparticles Through Lyophilization of ProteinPoly(ethylene glycol) Aqueous Mixture," Pharmaceutical Research, 17 (11), 1367.

Morita, T., Y. Sakamura, Y. Horikiri, T. Suzuki, and H. Yoshino. 2000b, "Protein Encapsulation into Biodegradable Microspheres by a Novel S/O/W Emulsion Method using Poly(ethylene glycol) as a Protein Micronization Adjuvant," J. Controlled Release, 69, 435.

Mosquiera, V. C. F., P. Legrand, H. Pinto-Alphandary, F. Puisieux, and G. Barratt. 2000. "Poly(D,L-lactide) Nanocapsules Prepared by a Solvent Displacement Process: Influence of the Composition on Physicochemical and Structural Properties," J. Pharmaceutical Sci., 89 (5), 614.

Mosqueira, V. C. F., P. Legrand, J.-L. Morgat, M. Vert, E. Mysiakine, R. Gref, J.-P. Devissaguet, and G. Barratt, G. 2001. "Biodistribution of Long-Circulating PEG-Grafted Nanocapsules in Mice: Effects of PEG Chain Length and Density," Pharmaceutical Research, 18 (10), 1411.

Nye, L., G.C. Forrest, H. Greenwood, J. J. Gardner, R. Jay, J. R. Roberts, and J. Landon. 1976. "Solid Phase Magnetic Particle Radioimmunoassay" Cli. Chim. Acta, 69, 387. 
O’Hagan, D. T., D. Rahmna, J. P. McGee, H. Jeffrey, M. C. Davies, P. Williams, S. S. Davis, and S. J. Challacombre. 1991. "Biodegradable Microparticles as Controlled Release Antigen Delivery Systems,” Immunology, 73, 239.

Okon, E., D. Pouliquen, D. Okon, Z.V. Stepanova, S.G. Lavit, B.N. Kudryavtsev, and P. Jallet. 1994. "Biodegradation of Magnetite Dextran Nanoparticles in the Rat, A Histologic and Biophysical Study,” Lab. Invest., 71(6), 895.

Oku, N., Y. Tokudome, H. Tsukada et al. 1995. "Real-time Analysis of Liposomal Trafficking in Tumor-Bearing Mice by use of Positron Emission Tomography," Biochimia et Biophysica Acta, 1238, 86 .

Olsvik, O., T. Popovic, E. Skjerve, K. Cudjoe, E. Hornes, J. Ugelstad, and M. Uhlén. 1994. "Magnetic Separation Techniques in Diagnostic Microbiology," Clinical Microbiology Reviews, 7,43 .

Pang, S. N. J. 1993. "Final Report on the Safety Assessment of Polyethylene Glycols (PEGs)-6, $-8,-32,-75,-150,14 \mathrm{M},-20 \mathrm{M}, ” J$. Am. Coll. Toxicol., 12, 429.

Papisov, M. I., V. Y. Savelyev, V. B. Sergienko, and V. P. Torchilin. 1987. "Magnetic Drug Targeting. Kinetics of Radiolabeled Magnetic Drug Carriers,” Int. J. Pharm., 40, 201.

Päuser, S., R. Resszka, S. Wagner, K-J Wolf, H.J. Buhr, and G. Berger. 1997. Superparamagnetic Iron Oxide Particles as Parker Substances for Searching Tumor Specific Liposomes with Magnetic Resonance Imaging. In: Scientific and Clinical Applications of Magnetic Carriers, pp 561-568, New York: Plenum Press.

Pouliquen, D., R. Perdrisot, A. Ermias, S. Akoka, P. Jallet, and J.J. Le Jeune. 1989. "SuperAramagnetic Iron Oxide Nanoparticles as Liver MRI Contrast Agent: Contribution of Microencapsulation to Improved Biodistribution,” Magn. Reson. Imag., 7, 619.

Pouliquen, D., J.J. Le Jeune, R. Perdrisot, A. Ermias, and P. Jallet. 1991. "Iron Oxide Nanoparticles for Use as an MRI Contrast Agent: Pharmacokinetics and Metabolism," Magn. Reson. Imaging, 9, 275.

Pouliquen, D. 2001. Magnetic Microparticles, A Historical Introduction. In: R. Arshady Microspheres Microcapsules and Liposomes (vol. 3). pp. 429-457, London, UK: Citus Books.

Quellec, P., R. Gref., L. Perrin, E. Dellacherie, F. Sommer, J. M. Verbavatz, and M. J. Alonso. 1998. "Protein Encapsulation within Polyethylene Glycol-coated Nanospheres. I. Physicochemical characterization,” J. Biomed. Mater. Res., 42, 45.

Regazzoni, A. E., M.A. Blesa, and A.J.G. Maroto. 1983. Journal of Colloid and Surface Science, 91, 560 . 
Robinson, P. J., P. Dunnill, and M.D. Lilly. 1973. "Properties of Magnetic Supports in Relations to Immobilized Enzyme Reactors,” Bioeng. 15 (3), 603.

Rosengart, A. J. 2001. The University of Chicago, Department of Neurology and Surgery, unpublished data.

Rudge, S. R., T. Kurtz, C. Vessely, L. Catterall, and D. Williamson. 2000. Biomaterials, 21, 1411.

Sako, M., and S. Hirota. 1986. "Embolotherapy for Hepatomas using Ferromagnetic Microspheres, its Clinical Evaluation and the Prospect of its Use as a Vehicle for ChemoemboloHyperthermic Therapy," Jpn. J. Cancer Chemother, part II, 13, 4, 1618.

Satoh, T., K. Kobayashi, S. Sekiguchi, and E. Tsuchida. 1992. "Characteristics of Artificial Red Cells, Hemoglobin Encapsulated in Poly-Lipid Vesicles,” ASAIO J, 38, M580 (1992).

Schütt, W., C. Grüttner, J. Teller, F. Westphal, U. Häfeli, B. Paulke, P. Goetz, and W. Finck. 1999. "Biocompatible Magnetic Polymer Carriers for Radionuclide Delivery," International Society for Artificial Organs, 23, 98.

Shah, S., K. Zhu, and C. Pitt. 1994. "Poly-DL-Lactic Acid: Polyethylene Glycol Block Copolymers. The influence of Polyethylene Glycol on Degradation of Poly-DL-Lactic Acid," J. Biomater. Sci. Polymer Edn. 5, 412.

Shelly, K., D. A. Feakes, M. F. Hawthorne, P. G. Schmidt, T. A. Krisch, and W. F. Bauer. 1992. "Model Studies Directed Toward the Boron Neutron-Capture Therapy of Cancer: Boron Delivery to Murine Tumors with Liposomes," Proc. Nat. Acad. Sci., 89, 9039.

Shinkai, M., M.Yanase, M. Suzuki, H. Honda, T. Wakabayashi, Jun Yoshidda, and T. Kobayashi. 1999. "Intracellular Hyperthermia for Cancer using Magnetite Cationic Liposomes," JMMM, 194, 176.

Slack, J. D., M. Kanke, G. H. Simmons, and P. Deluca. 1981. “Acute Hemodynamic Effects and Blood Pool Kinetics of Polystyrene Microspheres Following Intravenous Administration," J. Pharm. Sci 70, 660.

Splenlehauer, G., T. Verrecchia, Y. Archimbaud, D. Bazile, M. Marland and M. Veillard Stealth. 1994. PLA-PEG Nanoparticles: Biopharmaceutical and Biochemical Aspect, $3^{\text {rd }}$ Eu. Symp. Control. Drug Del.

Srdic, Winterer M., A. Möller et al. 2001. Nanocrystalline Zirconia Surface-Doped with Alumina: Chemical Vapor Synthesis, Characterization, and Properties, Journal of the American Ceramic Society 84, 2771. 
Stark, D. D., R. Weissleder, G. Elizondo, P. F. Hahn, S. Saini, L. . Todd, J. Wittenberg, and J. T. Ferrucci Jr. 1988. Superparamagnetic Iron Oxie: Clinical Application as a Contrast Agent for MR Imaging of the Liver, Radiology, 168, 297.

Stolnik, S., C. R. Heald, J. Neal, M. C. Garnett, S. S. Davis, L. Illum, S. C. Purkis, R. J. Barlow and P. R. Gellert. 2001. "Polylactide-Poly(ethyleneglycol) Micellar-like Particles as Potential Drug Carriers: Production, Colloidal Properties and Biological Performance," Journal of Drug Targeting, 9, 361.

Sun, X. C., A. Gutierrez, M. J. Yacaman et al. 2000. "Investigations on Magnetic Properties and Structure for Carbon-Encapsulated Nanoparticles of $\mathrm{Fe}, \mathrm{Co}, \mathrm{Ni}$," Materials Science and Engineering, A 286, 157.

Tanimoto, A. 2001. Magnetic Resonance Imaging with Superparamagnetic Nanocapsules. In: R. Arshady Microspheres Microcapsules and Liposomes, (vol. 3). pp. 525-558, London, UK: Citus Books.

Thomas, T. E., and P.M. Lansdorp. 1994. Progress in Clinical and Biological Research, 389, 65.

Thomassen, T., U. N. Wiggen, H. G. Gundersen, A. K Fahlivik, O. Aune, and J. Klaveness. 1991. "Structure Activity Relationship of Magnetic Particles as MR Contrast Agents," Mag. Reson. Imag., 9, 255.

Torchilin, V. A., M. I. Shtilman, V. . Trubetskoy, K. Whiteman, and A.M. Milstein. 1994. "Amphiphilic Vinyl Polymers Effectively Prolong Liposome Circulation Time," Biochim. Biophys. Acta, 1195, 181.

Torchilin, V. P., and V.S. Trubetskoy. 1995. "Which Polymers Can Make Nanoparticulate Drug Carrier Long Circulating?” Adv. Drug Delivery Rev. 16, 141-155.

Tsyb, A. F., I. S. Amosov, B. M. Berkovsky, V.I. Nikitina, M. M. Rozhinsky, L.V. Suloyeva, and G. M. Shakhlevich. 1983. "Magnetic Fluid at Contrast Media,” JMMM, 39, 183.

Van der Wolf, J. M., J. R. C. M. Van Beckhoven, Ph. M. de Vries, and J. W. L. Van Vuurde. 1994. Journal of Applied Bacteriology, 77, 160.

Van-Leengoed, H. L., V. Cuomo, A. A. Versteeg et al. 1994. " Fluorescence and Photodynamic Activity of Zinc Phtalocyanine Administered in Liposomes," British Journal of Cancer, 65, 840.

Weinmann, H. J., R. C. Brasch, W. R. Press, and G. E. Wesbey. 1984. "Characteristics of Gadolinium-DTPA Complex: A Potential NMR Contrast Agent,” Am. J. Roentgenol., 142, 619.

Weissleder, R., D. D. Stark, B. L. Engelstad, B. R. Bacon, C. C. Compton, D. L. White, P. Jacobs, and J. Lewis. 1989. "Superparamagnetic Iron Oxide: Pharmacokinetics and Toxicity," Am. J. Roentgenol, 152, 167. 
Weissleder, R., A. Bogdanov, E. A. Neuwelt, and M. Papisov. 1995. "Long Circulating Iron Oxides for MR Imaging,” Adv. Drug Deliv. Rev. 16, 321.

Wheldon, T. E. and J. A. O’Donoghue. 1990. “The Radiobiology of Targeted Radiotherapy," Int. J. Radiat. Biol., 58, 1990, 1-21.

Widder, K. J., A. E. Senyei, and D. G. Scarpelli. 1978. "Magnetic Microspheres: a Model System for Site Specific Drug Delivery ,” Proc. Soc. Exp. Biol. Med., 58, 141.

Yoshioka, T., M. Hashida, S. Muranishi, and H. Sezaki. 1981. "Specific Delivery of Mitomycin C to the Liver, Spleen and Lung: Nano- and Microsphereical Carriers of Gelain," Int. J. Pharm. 8, 131.

Yoshioka, H. 1991. "Surface Modification of Haemoglobin-Containing Liposomes with Polyethylene Glycol Prevents Liposome Aggregation in Blood Plasma," Biomaterials, 12, 861. 


\section{APPENDIX A: LARGE-ANIMAL EXPERIMENTS}

The general research approach of these experiments and a summary of results are presented in Section VI. Six monkeys (details below) were studied and injected (intravenously or intraarterially) with Magacell-polyethylene glycol (PEG) -500 particles (obtained from Cortex Biochem, Inc.). The individual particle size was reconfirmed by electron-microscopically and followed a Gaussian distribution ranging from less then 1 to maximally $20 \mu \mathrm{m}$; peak size distribution was found at $\sim 4.5 \mu \mathrm{m}$. A total of four intra-arterial upstream and three systemic venous particle injections were used. External magnetic trapping was performed at the thigh level of the femoral arteries first after intra-arterial and second after intravenous particle injections as well as over the external surface of exposed monkey brain after systemic, intravenous injections as detailed below. Separate magnetic sources were used (permanent $\mathrm{NdFeB}$ magnets, diameter 9 and $20 \mathrm{~mm}$; magnetic field strength $0.4 \mathrm{~T}$ at surface; Dexter Corporation Magnetic Technologies, Inc.). Details are provided in Figures A.1 through A.21.

\section{A.1 ARTERIAL TRAPPING}

Because of relatively small animal size (about $2 \mathrm{~kg}$ ) we used the relatively larger femoral artery as well as foot arteries as targets for externally guided particle trapping (Figure A.1). Small distal (feet) arteries range in size from 0.5 to $1.5 \mathrm{~mm}$ inner diameter.

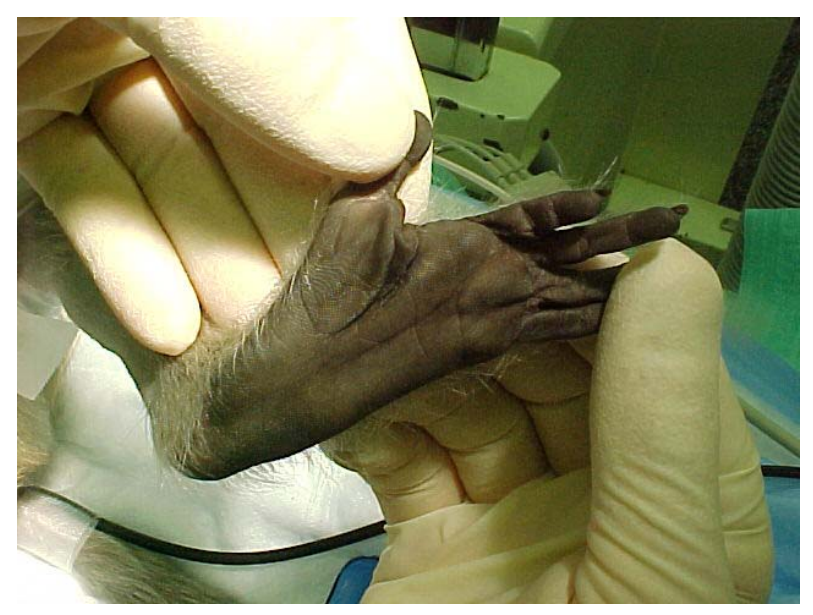

Figure A.1. Foot site in test animal for externally guided particle trapping. 
Figure A.2. Intra-arterial injection I.

Animal \#1:

Injection point \#1

Magnet \#1 only

Animal \#2:

Plus: Other side: Injection point \#2 and magnet \#2

Animal \#8:

Injection point \#2 and magnet \#2 only

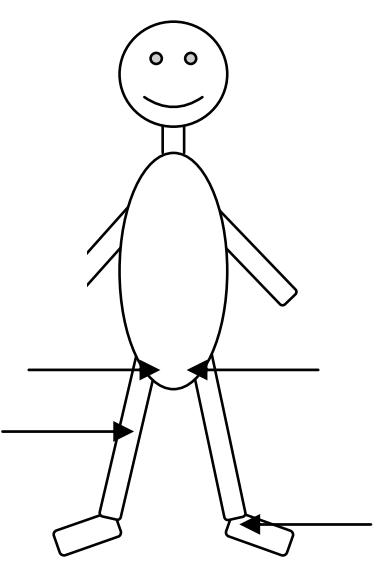

Figure A.3. Intra-arterial injection II.

Selective arterial catheterization (accessed in the axilla; see Figure A.2) were used to inject particles intra-arterially into the abdominal aorta, that is, upstream to the magnet.

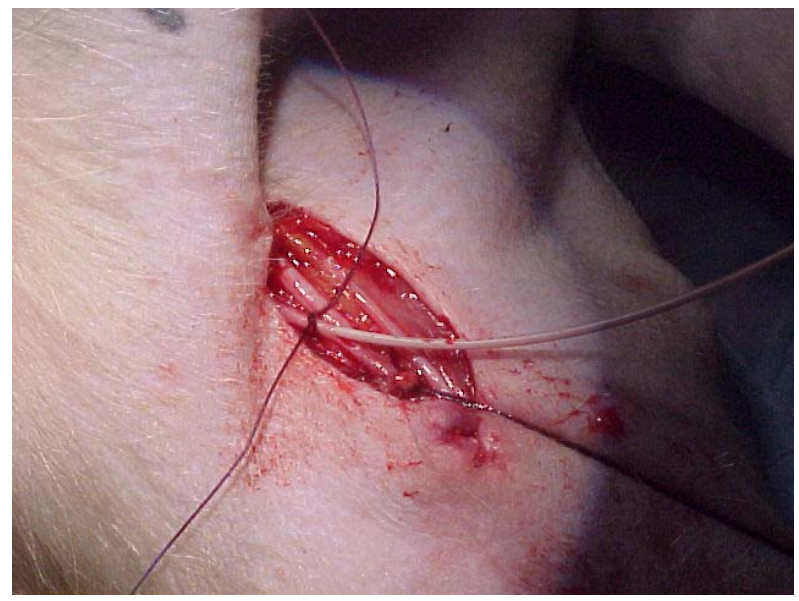

Figure A.4. Intra-arterial injection III.

Demonstration of the arterial monkey circulation and identification of catheter placement was done via $\mathrm{x}$-ray verification. Accumulated particles at either vascular occlusion or below the externally placed magnet were not identified on the standard animal fluoroscopy.

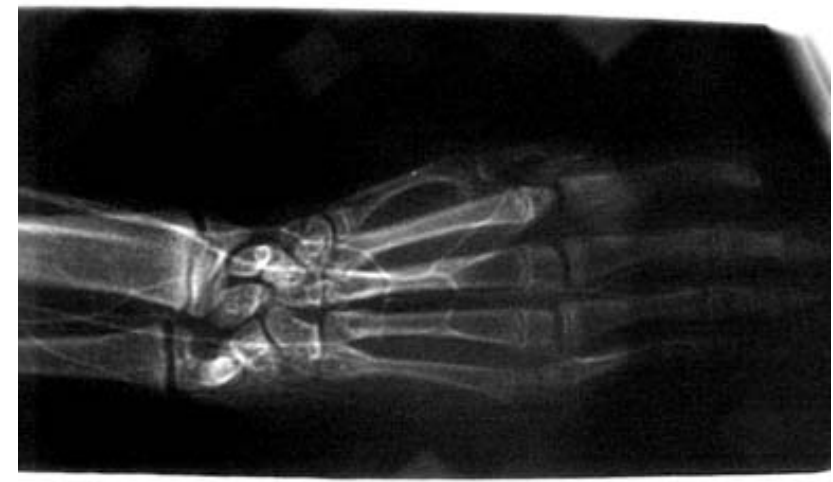


Figure A.5. Intra-arterial injection IV.

Animal \#1

Hematoxylin-eosin staining of a cross-section through the monkey foot directly under the site of external magnet placement. Twenty-five milligrams of particle were intra-arterially injected and an external magnet (9 $\mathrm{mm}$ in diameter) was placed over the foot surface. Magnetically trapped particles are identified as "black" accumulations, occluding two arterial branches.

Figure A.6. Intra-arterial injection V.

Animal \#1

Normal arterial vessels with no accumulation of magnetic particles were found in the contralateral, "control" artery, which did not have an external magnet applied (slightly longitudinal tissue section).

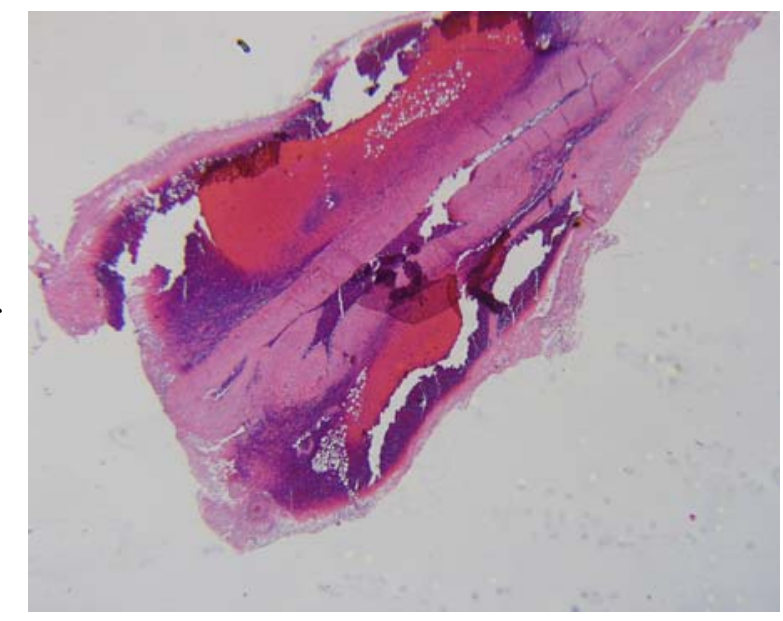

Figure A.7. Intra-arterial injection VI.

Animal \#2, left leg

Femoral intra-arterial particle injection $(25 \mathrm{mg})$ and magnet (9 $\mathrm{mm}$ diameter) applied above the foot artery.

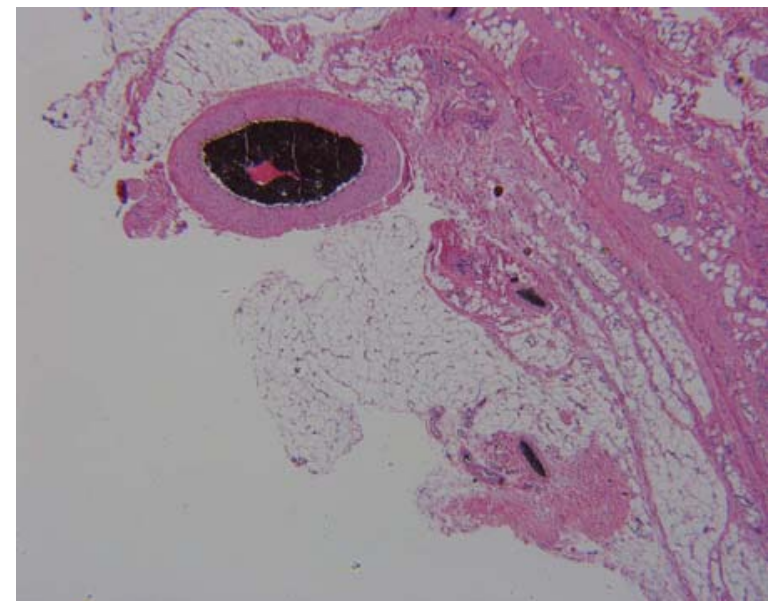


Figure A.8. Intra-arterial injection VII.

Animal \#2, right leg

Similar femoral intra-arterial particle injection (now $15 \mathrm{mg}$ ) and magnet $(9 \mathrm{~mm}$ diameter) applied above the femoral artery at thigh level. Again, histology identifies successful particle trapping in the arterial segment beneath the magnet despite an average blood flow mean velocity of about $90 \mathrm{~cm} / \mathrm{sec}$.

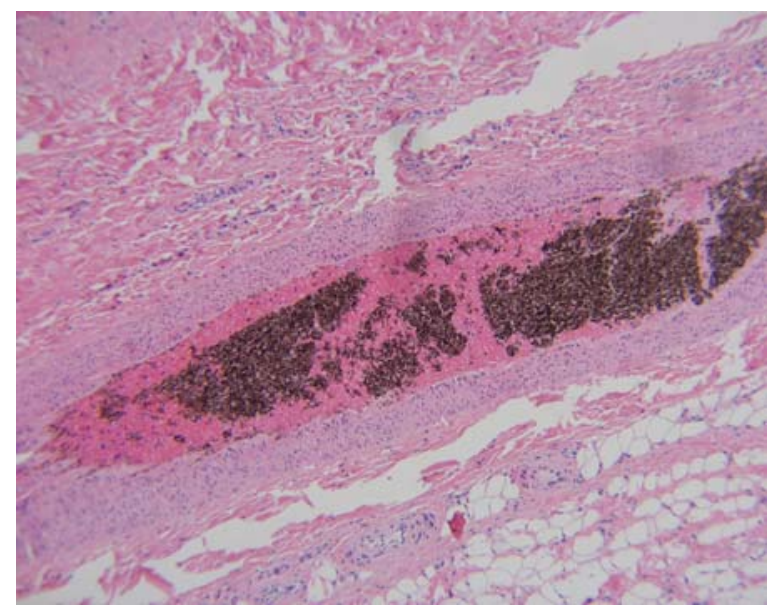

Figure A.9. Intra-arterial injection VIII.

Animal \#2, right leg

Magnetic particles can be identified in smaller blood vessels outside the direct circumferential area touched by the external magnet ("black" accumulations) $(9 \mathrm{~mm}$ magnet over thigh, femoral artery).

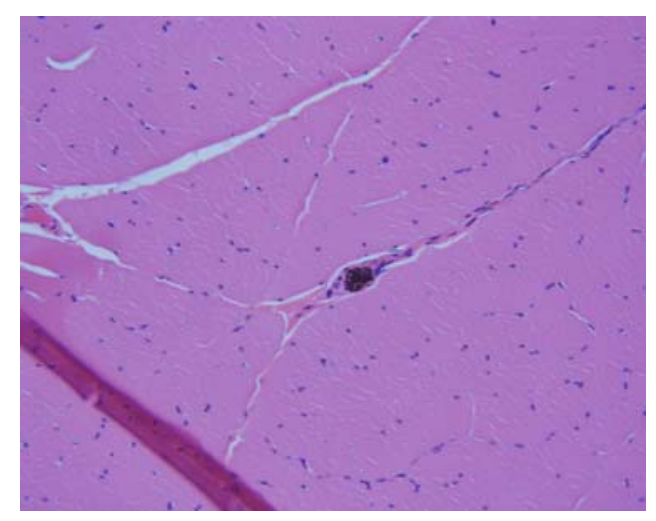

Figure A.10. Intra-arterial injection IX.

\section{Animal \#2, right leg}

Particles were also repeatedly identified outside the actual vascular lumen, as deposits into muscular tissue near the magnet, indicating particles leaving the arterial lumen and crossing the vascular wall, induced by magnetic force.

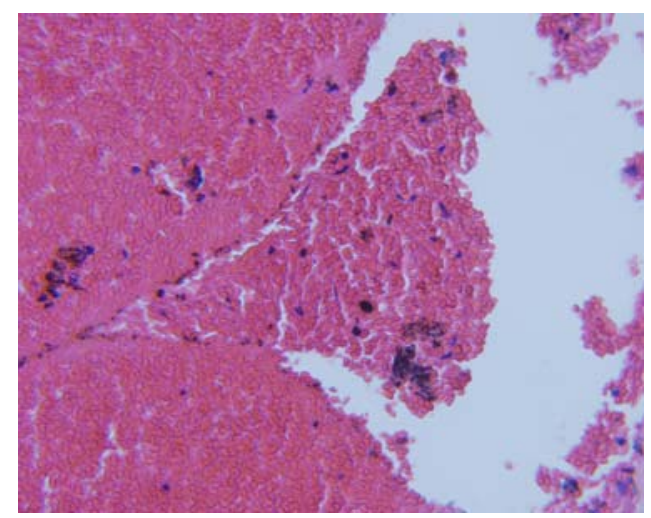


Figure A.11. Intra-arterial injection X.

Animal \#2, right leg

Histology: cross section of a "control" section at the level of the femoral artery but slightly below the actual magnet (farther downstream or more distally). The histological examination did not identify any particle accumulations.

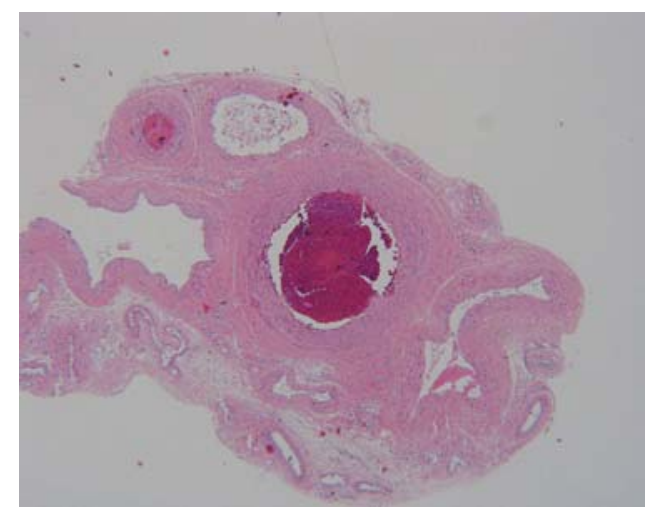

Figure A.12. Intra-arterial injection XI.

\begin{abstract}
Animal \#8
The histological specimen identifies successful particle trapping (dark accumulations within the vessel lumen) directly and only under the magnet. Particles $(25 \mathrm{mg}$ ) were injected directly into the artery farther upstream to the magnet (note: a $20-\mathrm{mm}$ magnet was used in this experiment).
\end{abstract}

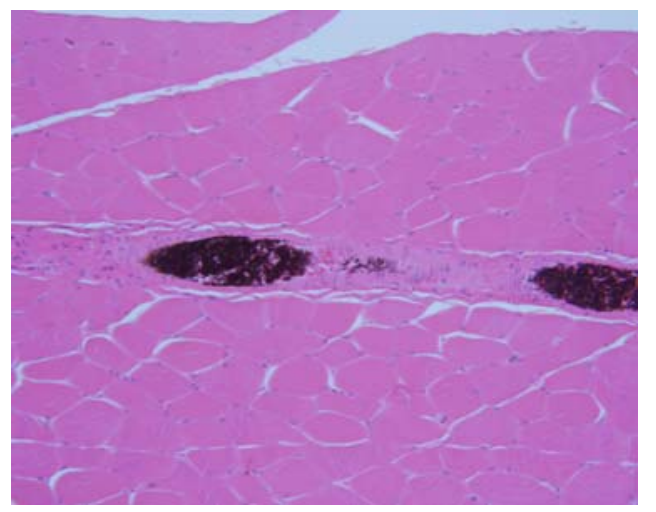

Figure A.13. Intra-arterial injection XII.

Animal \#8

Larger magnification of intra-arterial injection XI.

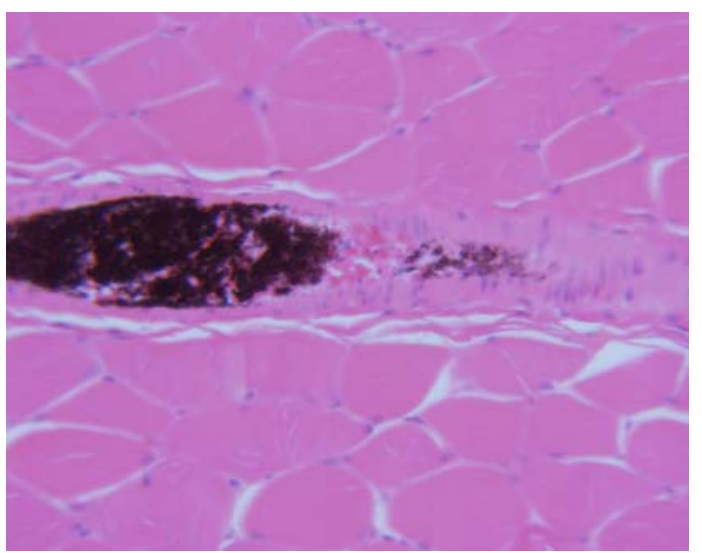




\section{A.1.1 Summary of Intra-Arterial Injection}

- In all previously described experiments, the magnetic particles were injected into the artery of a monkey via an intra-arterial catheter and several inches upstream to the magnetic trapping site.

- Variables studied included two different magnetic strengths and various injection dosages of particles as outlined above.

- All experiments identified successful trapping of magnetic particles within the tested arteries exactly, and exactly within the segment underlying the externally placed magnet (arteries studied were monkey foot and femoral arteries).

- Control sections, that is, arterial segments not underlying the external magnet, did not reveal any particle accumulations even when sections were taken close to the magnet position.

\section{A.2 INTRAVENOUS INJECTIONS}

Three animals were studied: In animal \#3 we injected systemically, that is, intravenously, $25 \mathrm{mg}$ magnetic particles, and animals \#4 and \#6 received each a total of $250 \mathrm{mg}$ particles intravenously. In all animals, a $9 \mathrm{~mm}$ magnet was placed at the right femoral artery (thigh level) prior to injection.

Unexpectantly, two animals expired within minutes after particle injection from respiratory failure (histology identified accumulation of particles within the lung leading capillary occlusions and consecutive respiratory failure as explained within the texts).

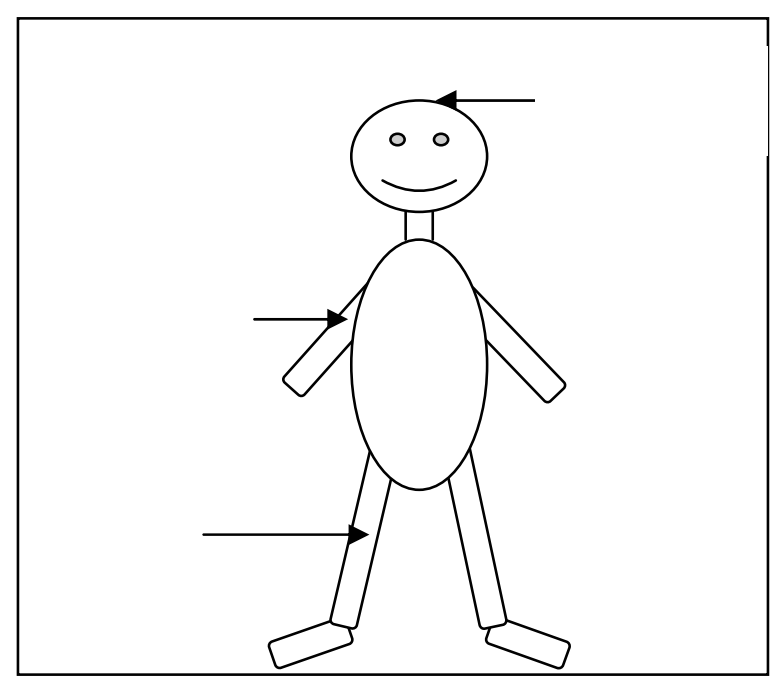

Figure A.14. General setup of magnet and venous injections site. 


\section{A.2.1. $\quad \underline{\text { Animal } 3}$}

This animal survived several minutes after slow, systemic injection of $25 \mathrm{mg}$ magnetic particles, enough time for the particles to circulate throughout the body and be trapped within the femoral artery, underneath a $9 \mathrm{~mm}$ magnet (see Figure A.15a). However, note that particle trapping leads to partial narrowing of the femoral artery but does not occlude the vessel completely as in the previous experiments. This is explained by a relatively low particle concentration reaching the arterial vasculature after venous injection as many particles are immediately trapped by the lung capillaries because of their relative large diameter ("first pass filtering effect" of the pulmonary vasculature; small, "dark" spots throughout the lung parenchyma; histology, Figure A.15b). Despite the deposition of particles throughout the lung tissue, this monkey survived with partially impaired respiratory function until euthanized 10 minutes after injection. Not shown is that the control femoral segment (without a magnet) taken from the opposite leg did not identify any particle trapping.

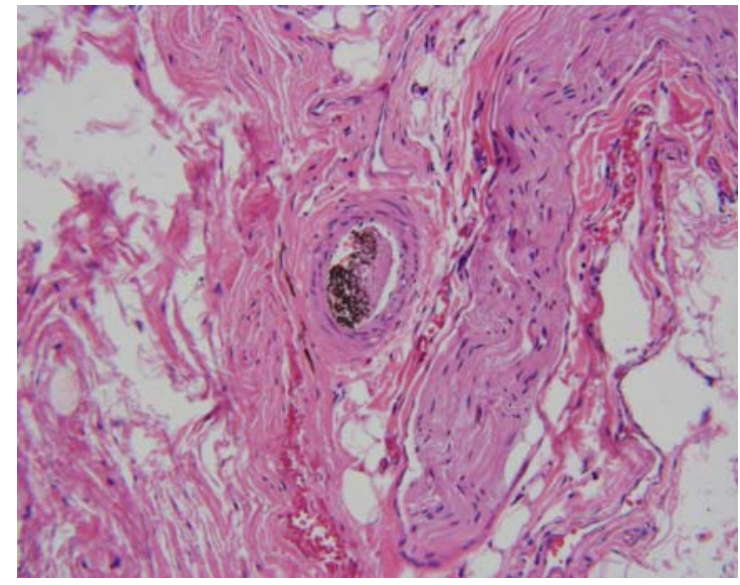

(a)

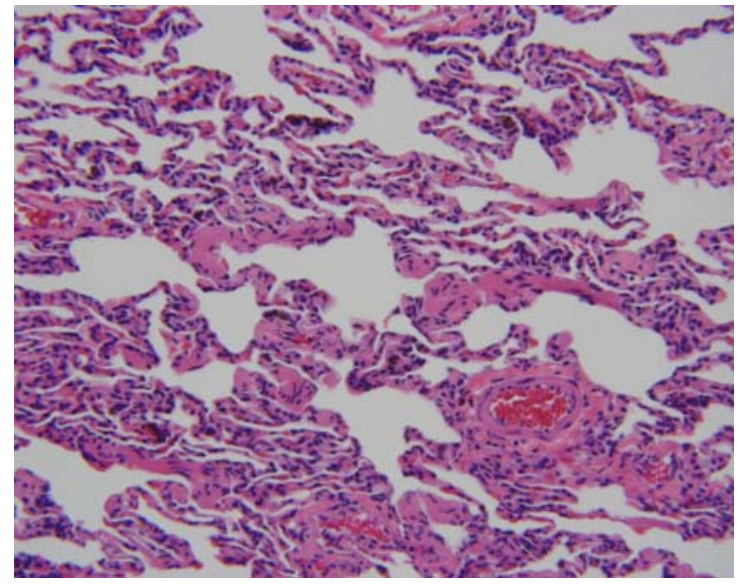

(b)

Figure A.15. Results of experiments on animal 3. (a) Microparticles trapped within femoral artery and (b) histology of the lung parenchyma. 


\section{A.2.2 $\underline{\text { Animal } 4}$}

In experiments on Animal 4, $250 \mathrm{mg}$ particles were injected intravenously and a $9 \mathrm{~mm}$ magnet was placed above the right femoral artery. Particle depositions (dark "dots" in Figure A.16a) were identified on histology. The animal died after four minutes from respiratory failure and showed even more marked particles occluding lung capillaries and hence inhibiting gas exchange (dark spots" on lung histology, Figure A.16b). Not shown is that the control femoral segment (without a magnet) taken from the opposite leg did not identify any particle trapping.

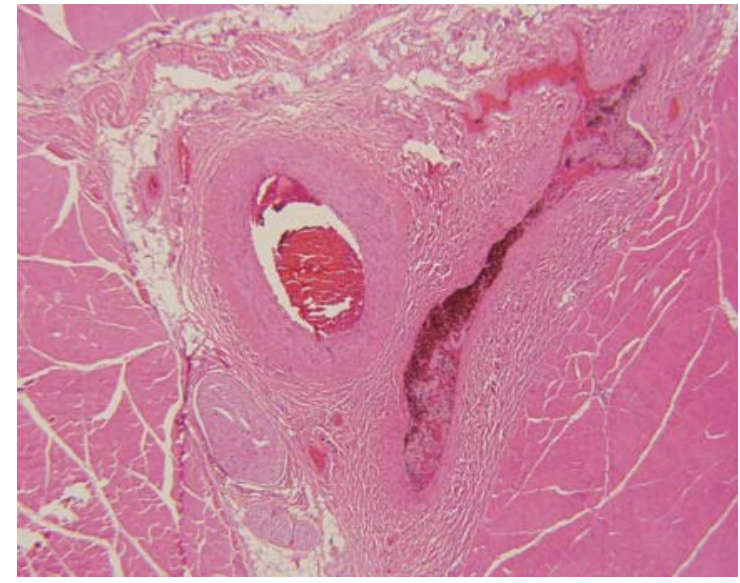

(a)

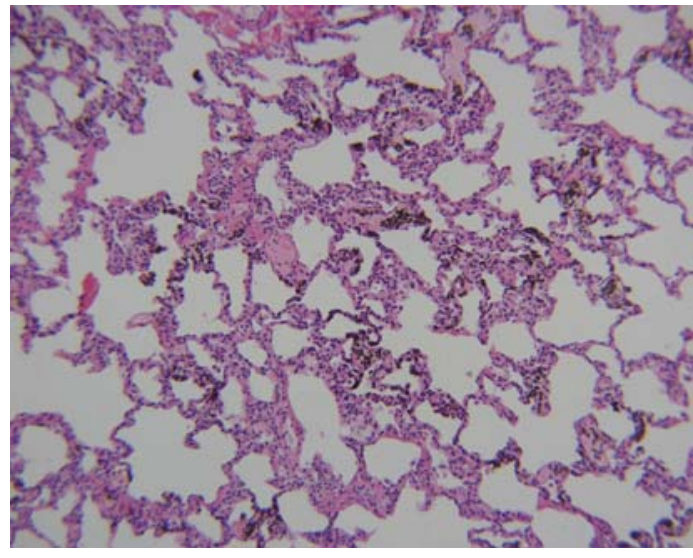

(b)

Figure A.16. Results of experiments on animal 4. (a) Particle depositions and (b) lung histology.

\section{A.2.3 $\underline{\text { Animal } 6}$}

Injection of $250 \mathrm{mg}$ particles intravenously after particles were buffered in $2 \% \mathrm{Na}_{4} \mathrm{P}_{2} \mathrm{O} 0.10 \mathrm{H}_{2} \mathrm{O}$ (MW 453.6g) and insonicated immediately prior to the injection to avoid particle accumulations. We tested whether particle agglomerations rather then actual individual particle sizes were responsible the lung capillary occlusions (the buffer injectate and insonication prior to injection minimize the occurrence of particle agglomeration; Figure A.17a). However, despite this preparation of the injectate, the monkey expired from lung failure within 5-1/2 minutes, after slow injection of only $65 \%$ of the total particle volume ("dark spots" in Figures A.17b and A.17c). Electron microscopy identified many particles larger than $5 \mu \mathrm{m}$ (the diameter of most capillaries) being responsible for the observed phenomenon. Some particles were, however, still trapped within the femoral artery underneath the $9 \mathrm{~mm}$ magnet. 


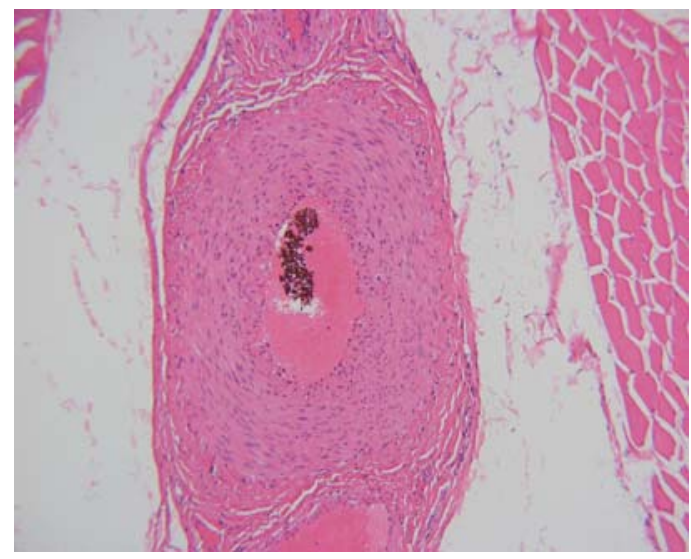

(a)

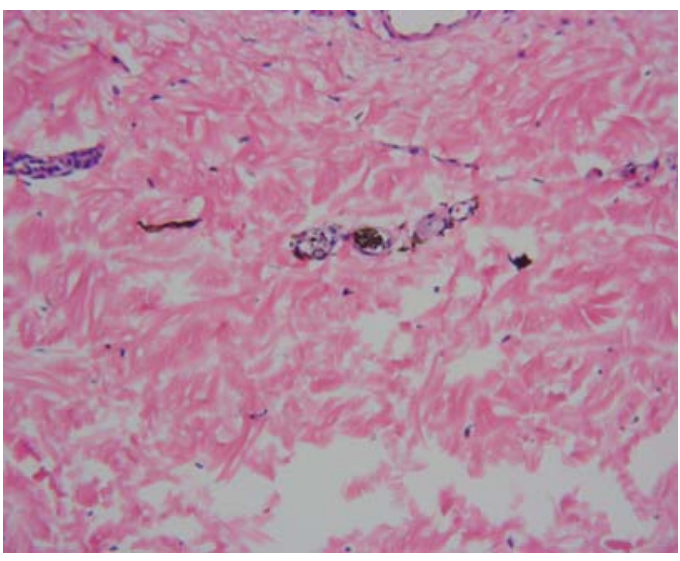

(b)

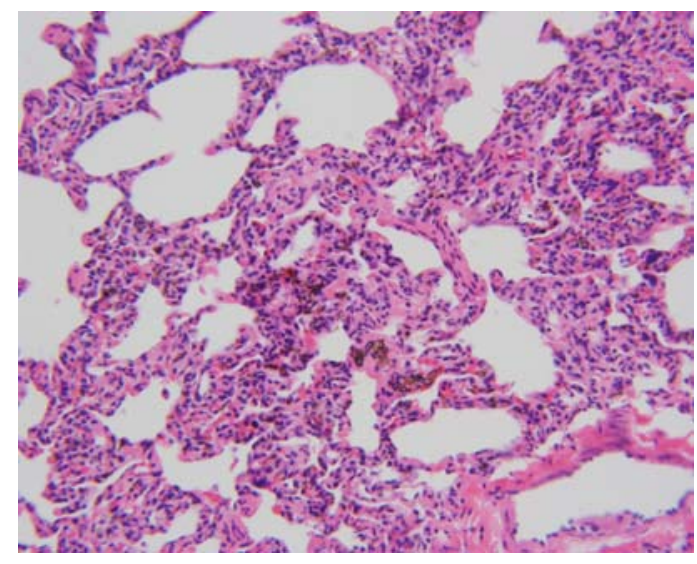

(c)

Figure A.17. Results of experiments on animal 6. (a) Cross-section of leg artery and partial filling of arterial lumen with magnetic particles (black), (b) higher magnification, and (c) lung parenchyma histology.

\section{A.2.4 Summary of Intravenous Injection Experiments}

- The magnetic particles were injected into a vein of a monkey; before these particles reach the arterial system, they have to pass the lung capillary system.

- In all three animals studied, marked particles deposition leading to capillary lung occlusion and consecutive respiratory failure of the animals were identified clinically and by histology.

- Lung capillary occlusions were due to particle-size polydispersity. Particles larger than about $5 \mu \mathrm{m}$ will occlude any capillary system, and the animals died of lung failure as this is the first capillary system that intravenously injected particles must pass.

- Importantly, magnetic particles able to circulate throughout the arterial systems (presumably sized in diameter less then $5 \mu \mathrm{m}$ ) were still trapped in femoral artery (as well as brain arteries, see next section).

- Control arterial sections, that is, arterial segments not underlying the external magnet, did not identify any particle accumulations. 


\section{A.3 INTRAVENOUS INJECTIONS - PARTICLE TRAPPING IN BRAIN ARTERIES}

The general set-up of these experiments is identified in Figure A.18. The magnet (20 mm in diameter) is placed directly on the monkey brain, leaving the brain "skin" (dura) intact but removing the skull bone (Figure A.19). The injection sites are venous, as in the experiments described in Section A.2.

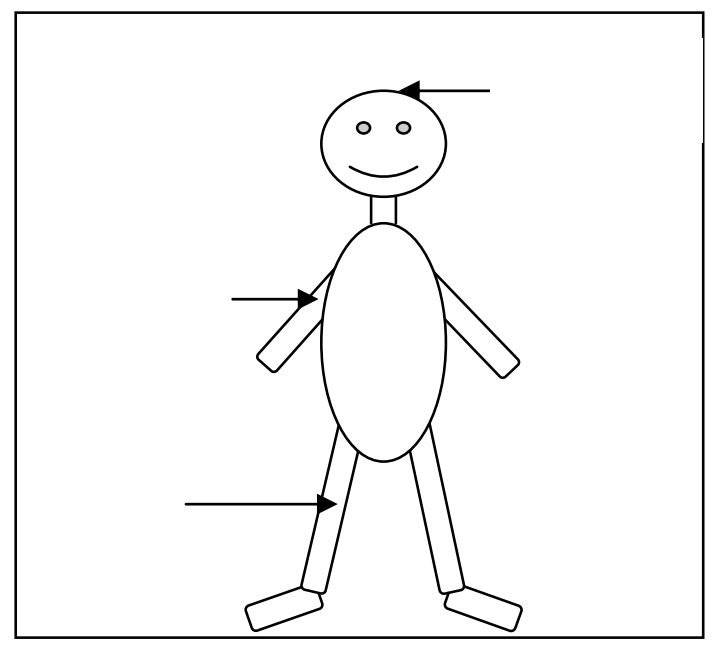

Figure A.18. General setup of experiments for intravenous injections, particle trapping in brain arteries.

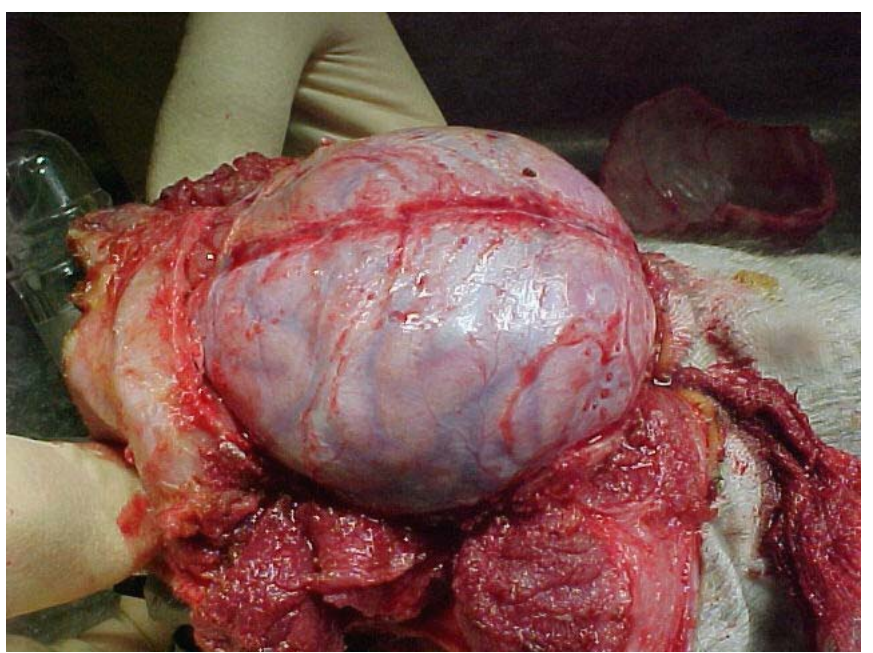

Figure A.19. Magnet placement for experiments for intravenous injections, particle trapping in brain arteries. 


\section{A.3.1 $\quad \underline{\text { Animal } 3}$}

The animal was injected intravenously with $25 \mathrm{mg}$ magnetic particles and euthanized after 10 minutes. Note that, as described in Sections A.1 and A.2, after intravenous injections most particles were "filtered out" by the lung capillaries and only small amounts of particles reached the brain arteries. Despite the relatively low arterial particle concentration reaching the animal organs, slight particle depositions are identified selectively in brain arteries directly underneath the magnet location.

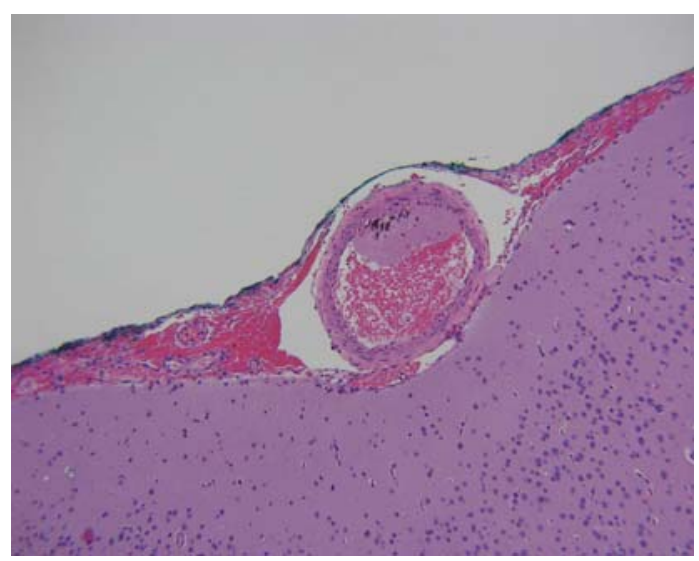

Figure A.20. Particle deposits directly under magnet in brain arteries of Animal 3.

\section{A.3.2 $\underline{\text { Animal } 6}$}

The animal was injected intravenously with $250 \mathrm{mg}$ magnetic particles (10 times the dose used for Animal 3) and died from lung failure after only 5-1/2 minutes. Particle deposition in brain arteries directly underneath the magnet location is identified in Figure A.21.

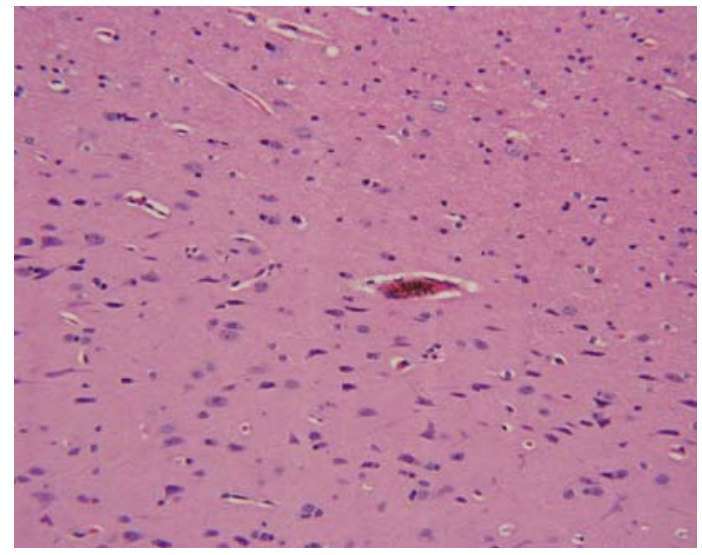

Figure A.21. Histology of experiments on animal 6. 


\section{A.3.3 Summary of Intravenous Injection and Particle Trapping in Brain Arteries}

- The magnetic particles were injected into a vein of a monkey; before these particles reach the arterial system, they have to pass the lung capillary system.

- In contrast to the previous experiments (Section A.2), we placed an additional magnet at the exposed brain's surface to investigate particle trapping at these sites.

- As expected, marked particle deposition occurred, leading to capillary lung occlusion and respiratory failure and death of the animals.

- In the two animals studied, particles were successfully trapped at brain arteries underneath the external magnet.

- Control sections of the opposite brain (no magnet positioned) showed no particle deposition. 


\section{APPENDIX B: CLOT LYSIS RESEARCH GOALS}

Our overall research goal is to advance the safety and effectiveness of acute ischemic stroke therapy by improving the benefit/risk ratio of tPA thrombolysis and hence the long-term outcome of acute ischemic stroke victims. The overall approach is the development of a novel tissue plasminogen activator (tPA) delivery system based on tPA loaded magnetic particles guided directly to the site of vascular occlusion by external magnetic fields. Such a tPA delivery system would conveniently combine the advantages of both intravenous (systemic) and intraarterial (catheter-facilitated) thrombolysis: noninvasiveness as the magnetic tPA carriers can be injected intravenously, and targeted as drug delivery is magnetically guided to and tPA focally released at the vascular clot to induce lysis. The focus of the proposed research is the necessary fundamental bioengineering step: the development and design of well characterized, biocompatible, functionally active, tPA loaded (encapsulated) magnetic nano- and microparticles able to induce effective thrombolysis. If successful, such tPA encapsulated magnetic particles can then be implemented in future research efforts to develop magnetic guidance systems for noninvasive, yet targeted stroke therapy. To be suitable for future human applications, our experimental approach, based on nanoscale technology, is to synthesize the magnetic tPA carrier according to specific predefined outcome metrics: monodisperse population in a size range of $100 \mathrm{~nm}$ to $3 \mu \mathrm{m}$, nontoxic, with appropriate magnetic properties, and demonstrating successful in vitro clot lysis.

The central building block of this drug delivery strategy are magnetic nano- and microparticles. These designer composites consist of a polymer-based matrix imbedded with nanocrystalline iron oxide $\left(\mathrm{Fe}_{3} \mathrm{O}_{4}\right.$ or $\left.\gamma-\mathrm{Fe}_{2} \mathrm{O}_{3}\right)$ crystals permitting the particles to orient themselves in magnetic fields, that is, to have 'paramagnetic' properties. Based on already existing evidence and our own experience, we propose that such magnetic particles can (a) be designed to have appropriate pharmacokinetic properties, i.e., vascular survival for several hours, (b) encapsulate proteins, such as IPA, into the particle matrix creating spheres suitable for magnetically-guided transport of "shielded" tPA, and c) release active tPA at the target site, either spontaneously or facilitated by an external "trigger" signal, to accomplish clot lysis. For the successful design of such tPA encapsulated magnetic particles, we intend a stepwise approach.

Specific Aim 1 will test two alternative methods to synthesize tPA encapsulated magnetic particles based on specific outcome metrics set in place to guarantee suitability for future magnetic guidance and use in humans.

Specific Aim 2 will establish stable release kinetics of tPA from the particle encapsulation, reconfirm the functional activity of released tPA, and investigate the use of ultrasound exposure to trigger tPA release.

Specific Aim 3 will investigate the in vitro clot lysis efficiency of the newly designed particles and compare thrombolysis rates of conventional tPA with tPA released from encapsulation.

Of note, in order to reduce overall research costs, we will first use a substitute protein, bovine serum albumin, to optimize particle synthesis and release parameters and then confirm the 
experimental results using tPA. If successful, these experiments will provide the foundation for a novel technology for active and targeted thrombolysis. 


\section{APPENDIX C: CANCER THERAPY RESEARCH GOALS}

The broad, long-term objective of this concept is to treat cancer patients whose current therapy choices are severely limited by tumor inaccessibility, inoperability, local and/or lymph node infiltration, and risk of toxicity to nearby organs. Our approach is to direct magnetic microspheres (MMS) loaded with chemo- or radiotherapeutic drugs to a predetermined tumor, where they perform a defined action (e.g., drug release, physical interaction with cells), and then disappear without a trace (biodegradation, excretion) and without harming the body (nontoxicity). Many difficult to treat tumors at various organ sites are potentially amenable to this therapy, for example, head and neck, pancreas, liver, kidney, and stomach cancer. Successful magnetic targeting could benefit about $15 \%$ of the 1.2 million new cancer patients per year in the United States alone. Furthermore, if successful, such an approach could be employed to combat additional cancers such as melanoma, breast and prostate cancer, either as a new treatment strategy or in combination with existing treatment strategies.

The specific aims of this research are (1) to identify microparticle behavior using two animal cancer models of head and neck cancer and several different tumor locations, (2) to develop optimal microspheres for magnetically assisted cancer radiotherapy in terms of size, best suspension flow behavior and maximum guidance/trapping, biodegradability/ compatibility, nontoxicity, magnetization, and radioactive loading, and (3) to develop and validate a clinical approach to treating head and neck cancer in an animal model using VX-2 tumor cells.

The research will encompass the investigation of magnetic targeting in both a nude mouse and a rabbit tumor model, the optimization and integration of all components necessary for MMS delivery to a tumor, and the testing and fine-tuning of the system in an H\&N animal tumor model in preparation for clinical trials. In order to achieve the research goals, magnetic radiolabeled microspheres will be used to quantitatively analyze the biodistribution of MMS after magnetic tumor targeting. The data will then be incorporated into a coupled fluid (blood) flow and magnetic field model to provide a tool for optimizing clinical treatment. 
Distribution for ANL-03/28

Internal (Printed and Electronic Copies):

P. Caviness

F. Chang

H. Chen

T. Chien

G. Crabtree

J. Eastman

M. Finck

P. Fischer

A. N. Ghebremeskel

M. D. Kaminski (20)

K. Kasza

L. Nuñez

Internal (Electronic Copy Only):

M. R. Hale, TIS

D. Lewis

External (Electronic Copies Only):

M. A. Buckley, ANL Library-E

E. Sackett, ANL Library-W

Chemical Engineering Division Review Committee Members:

H. U. Anderson, University of Missouri-Rolla, Rolla, MO

C. L. Hussey, University of Mississippi, Oxford, MS

M. V. Koch, University of Washington, Seattle, WA

V. P. Roan, Jr., University of Florida, Gainesville, FL

J. R. Selman, Illinois Institute of Technology, Chicago, IL

J. S. Tulenko, University of Florida, Gainesville, FL

A. J. Rosengart, The University of Chicago, Chicago, IL

U. Häfeli, The Cleveland Clinic Foundation, Cleveland, $\mathrm{OH}$

L. Johns, The University of Chicago, Chicago, IL

L. Macdonald, The University of Chicago, Chicago, IL

P. Pytel, The University of Chicago, Chicago, IL

Y. Xie, The University of Chicago, Chicago, IL 